\title{
A revision of the genus Rudolfina Roháček (Sphaeroceridae: Limosininae)
}

\author{
Steven Mark PAIERO ${ }^{1, *} \&$ Stephen A. MARSHALL ${ }^{2}$ \\ ${ }^{1,2}$ University of Guelph Insect Collection and Insect Systematics Laboratory, School of Environmental \\ Sciences, University of Guelph, Guelph, Ontario, N1G 2W1, Canada. \\ *Corresponding author: paieros@uoguelph.ca \\ ${ }^{2}$ Email: samarsha@uoguelph.ca \\ ${ }^{1}$ urn:1sid:zoobank.org:author:B172670C-2159-4D6B-9542-53406F315DC7 \\ ${ }^{2}$ urn:lsid:zoobank.org:author:0BBC95DD-0E89-49DC-80BE-E40261E9F5FE
}

\begin{abstract}
The genus Rudolfina Roháček, 1987 is revised and redefined with the description of the following nine new species, all from the New World: R. bucki sp. nov. (Mexico), R. exuberata sp. nov. (widespread, from USA to Brazil), R. howdeni sp. nov. (Mexico), R. megepandria sp. nov. (Mexico), $R$. newtoni sp. nov. (Mexico), R. pauca sp. nov. (Guatemala, Mexico), R. pilosa sp. nov. (Mexico), $R$. remiforma sp. nov. (Mexico) and $R$. tumida sp. nov. (Mexico, USA). Rudolfina is compared to closely related genera in the Archiceroptera genus complex, which in turn is recognized as part of a large, mostly Neotropical clade including Robustagramma Marshall \& Cui, 2005, Pterogramma Spuler, 1924, Aptilotella Duda, 1924, Bitheca Marshall, 1987, Bromeloecia Spuler, 1924 and Archiceroptera Papp, 1977.
\end{abstract}

Keywords. Sphaeroceridae, Rudolfina, Limosininae, Nearctic, Neotropical.

Paiero S.M. \& Marshall S.A. 2020. A revision of the genus Rudolfina Roháček (Sphaeroceridae: Limosininae). European Journal of Taxonomy 593: 1-48. https://doi.org/10.5852/ejt.2020.593

\section{Introduction}

The genus Rudolfina Roháček, 1987 was introduced as a new name for the pre-occupied Rudolfia Roháček, 1982, which was originally described for a single Palaearctic species, Limosina rozkosnyi Roháček, 1975. Three species have since been described: R. digitata Marshall, 1991 and $R$. cavernicola Marshall \& Fitzgerald, 1997 from North America (Marshall 1991; Marshall \& Fitzgerald 1997) and R. zhangi Su, 2017 from China (Su et al. 2017). Marshall (1982) also recognized several undescribed species of Rudolfina s. str. from Mexico, but deferred publishing descriptions of those species until adequate material was available to properly treat the Neotropical fauna and to determine the limits between Rudolfina and superficially similar Neotropical species in the Archiceroptera genus complex.

We here redefine the genus Rudolfina and describe nine new species following the examination of approximately 2000 specimens of Rudolfina and over 6000 specimens of other genera in the Archiceroptera genus complex. 
Rudolfina is diagnosed by the following characters: (1) mid tibia with 1 proximal anterodorsal seta, 1 medial anterodorsal seta, 1 distal anterodorsal seta and 1 distal posterodorsal seta; (2) costa with 1 well-developed costagial seta; (3) male sternite 5 posteromedially emarginate with darkened lobe or process on each side of the emargination; (4) female tergite 8 tripartite, middle part articulating or fused with the epiproct; (5) epiproct medially weakened; (6) female cercus strap-like, with strong, flattened apical seta; and (7) female abdomen with paired bisetose sclerites posterior to sternite 8. All Rudolfina except $R$. cavernicola have the female cercus partially fused with the epiproct. Most of these diagnostic characters are also defining characters that support the genus Rudolfina, as defined here, as a monophyletic group. The strongest synapomorphies for the genus are the characters of the female terminalia listed above (characters 4-7), along with the costagial seta (character 2) and the male sternite 5 (character 3$)$.

\section{Material and methods}

\section{Collection and storage}

Most specimens were collected into soapy water, transferred to alcohol for storage, and later dried and point-mounted. Abdomens of some specimens were removed and cleared in hot $10 \%$ potassium hydroxide for 6-10 minutes before being neutralized with $10 \%$ acetic acid for 10 minutes, rinsed in deionized water, and then placed in glycerin for examination. Dissected genitalia were stored in glycerinfilled microvials pinned below the specimens.

\section{Specimen data}

All label data are presented in a consistent manner, not verbatim from the labels; in a few cases, obvious spelling errors were corrected. Short-forms or abbreviations used on specimen labels are normally interpreted and given in full. Elevations and geographical coordinates are given only if present on the original label. All specimens were given unique identifiers (debu followed by an 8-digit number) and their collection data were captured within the University of Guelph Insect Collection database; these are not repeated in the text except for holotypes or for image reference. The specimen data will ultimately be hosted on Canadensys (https://www.canadensys.net/). Collection data for paratypes and other specimens examined were organized alphabetically by country, state/province, and locality name. Distribution maps are given for all New World species (Fig. 21) and were generated using SimpleMappr (Shorthouse 2010). The abbreviation FIT is used to indicate 'flight intercept trap'.

\section{Terminology}

The terminology for external morphology largely follows Cumming \& Wood (2010) with a few modifications given below; terminology for male and female genitalia follows Smith \& Marshall (2004) with modifications from Cumming \& Wood (2010). Figures 1-4 illustrate head chaetotaxy, wing venation, and male and female genitalia. Seta(e) and setula(e) are large and small (respectively) socketed macrotrichia. The $\mathrm{CuA}_{1}$ and $\mathrm{M}_{1}$ stub veins are the short portions of these veins that project distally beyond cell $\mathrm{dm}$. The subanal plate connects the posteroventral angles of the epandrium. The female abdomen has a pair of small bisetose sclerites posterior to sternite 8 . These bisetose sclerites are homologous with the posterior portion of sternite 8 in related taxa (e.g., Pterogramma Spuler, 1924 and Robustagramma Marshall \& Cui, 2005; see Smith \& Marshall 2004 and Marshall \& Cui 2005) and reflect a general desclerotization of sternite 8 , leaving a distinct transverse anterior portion and the paired posterior sclerites.

\section{Measurements}

Body length was measured from the anterior portion of the frons to the tip of the abdomen. Measurements were made using a Leica M2 125 with an ocular micrometer. 


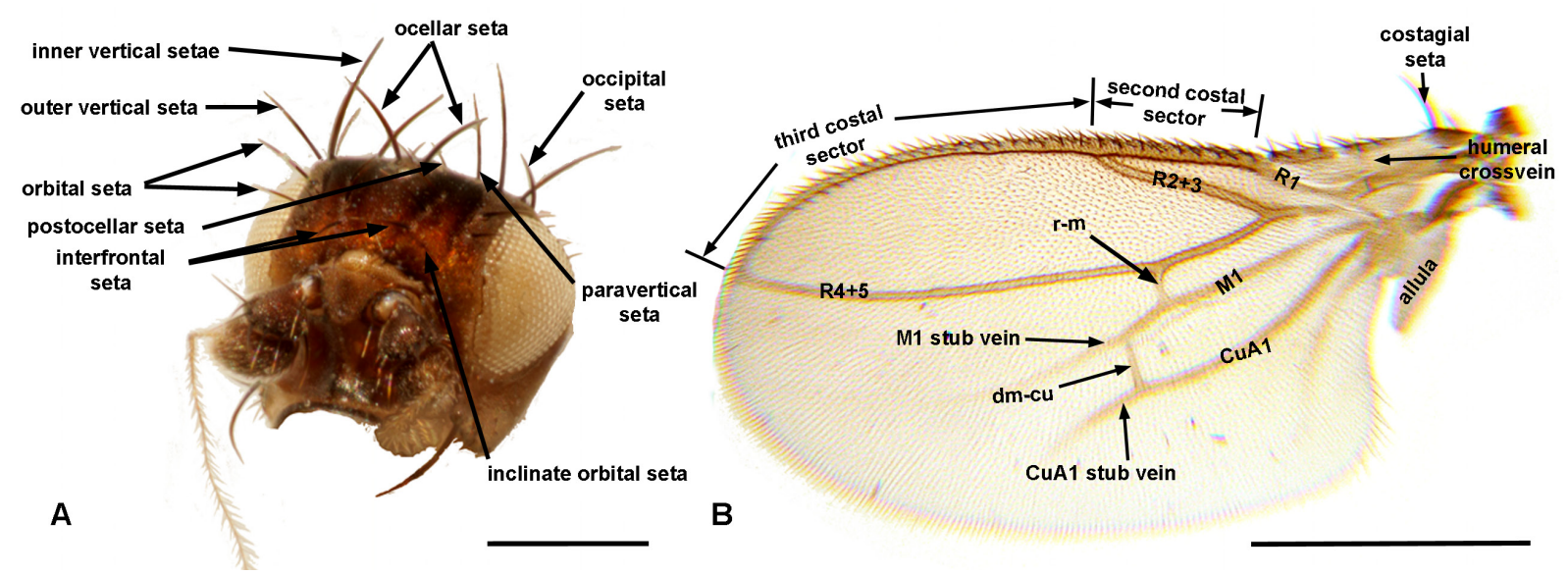

Fig. 1. A. Rudolfina howdeni sp. nov., head (debu01086104). B. R. exuberata sp. nov., wing (debu00276674). Scale bars: A $=0.20 \mathrm{~mm}$; $=0.35 \mathrm{~mm}$.

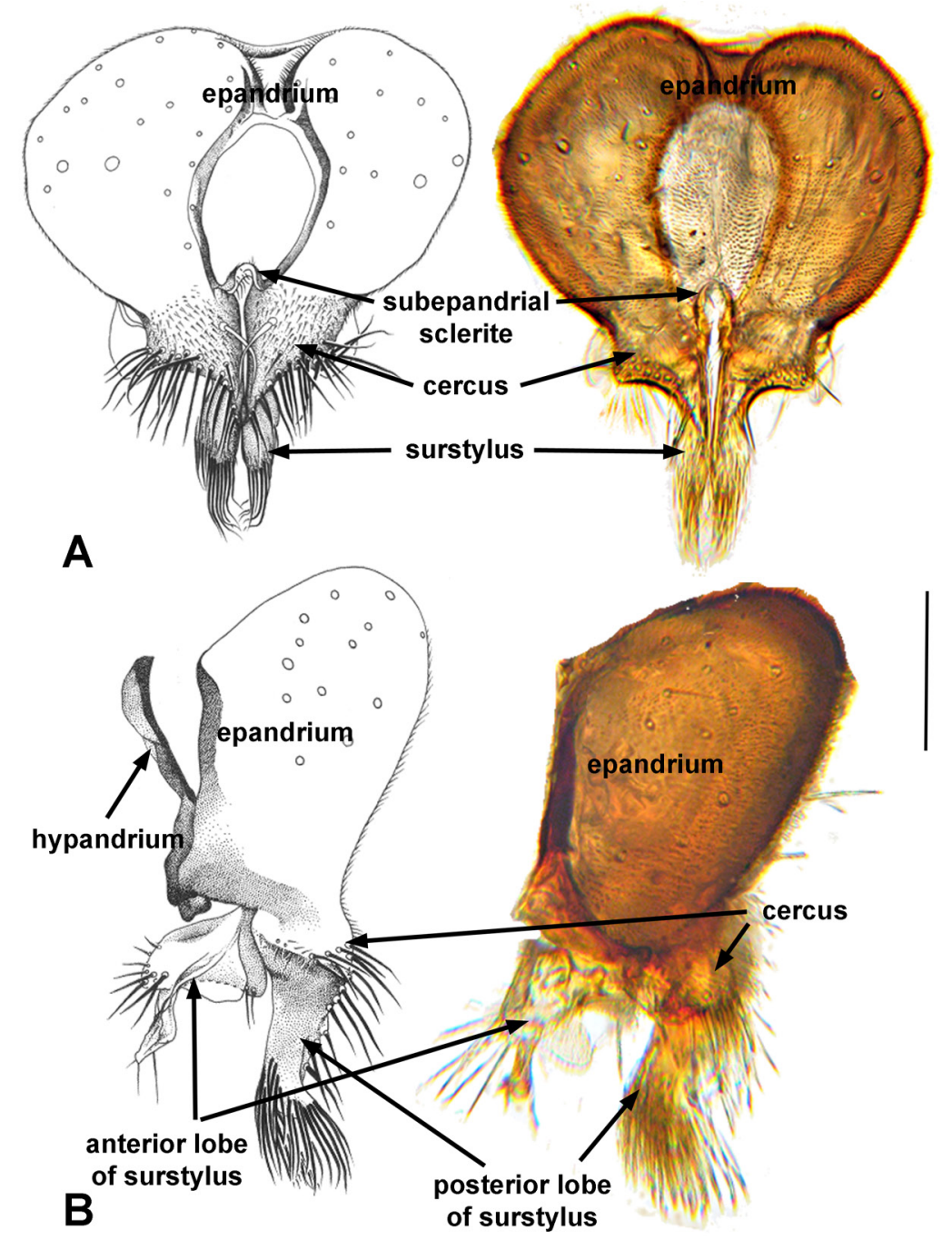

Fig. 2. Rudolfina tumida sp. nov. A. Male epandrium, cerci and surstyli, posterior view. B. Male epandrium, cercus and surstylus, lateral view. Illustrations and photographs from debu01086083. Scale bar: $\mathrm{A}-\mathrm{B}=0.10 \mathrm{~mm}$. 


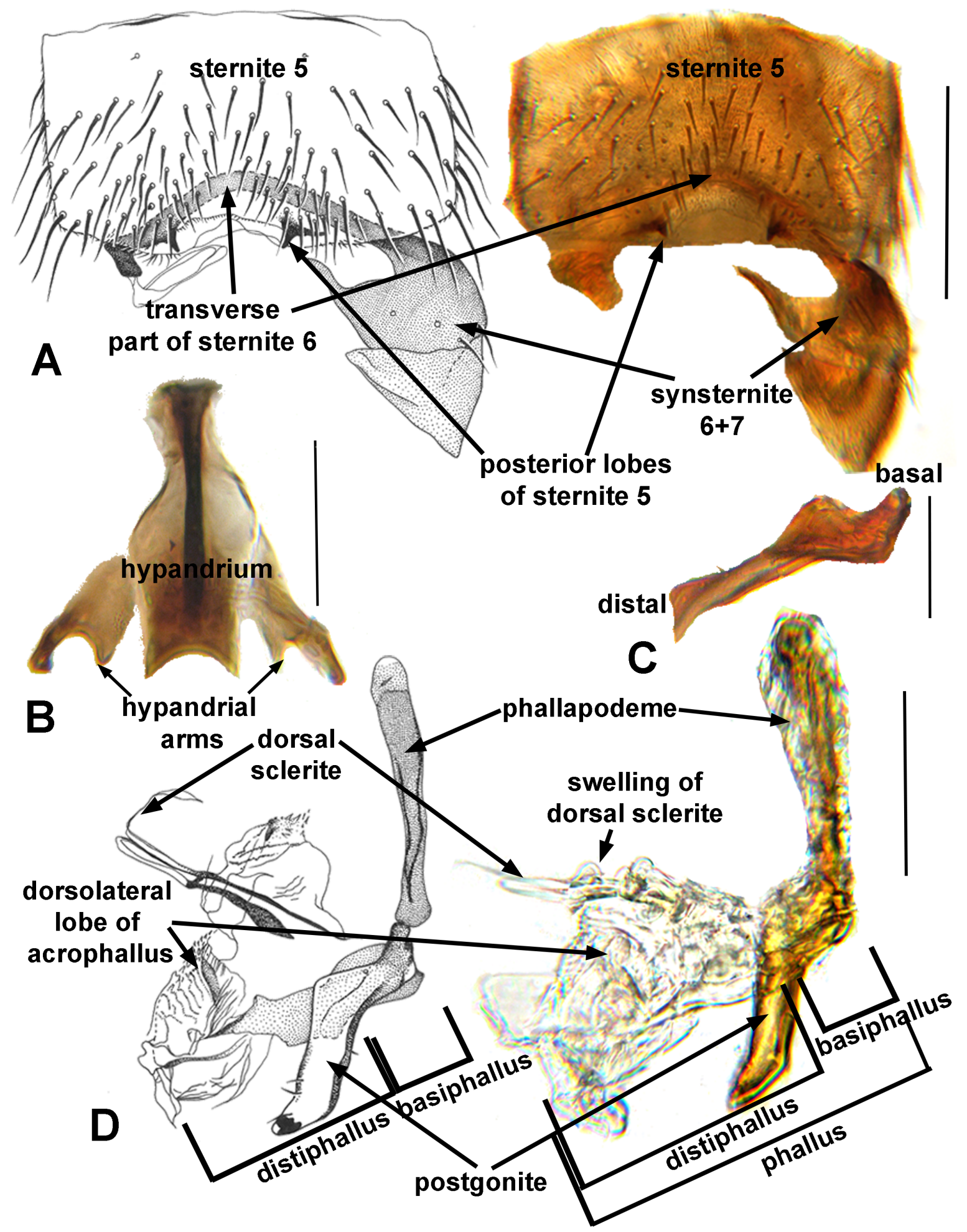

Fig. 3. Male morphology. A. Rudolfina tumida sp. nov., sternite 5, ventral view (debu01086083). B. R. exuberata sp. nov., hypandrium (debu00242299). C. R. cavernicola Marshall \& Fitzgerald, 1997, postgonite, lateral view (debu01086077). D. R. tumida sp. nov., phallus (including the basiphallus and distiphallus), postgonite and phallapodeme, lateral view (debu01086083). Scale bars: $\mathrm{A}=0.15 \mathrm{~mm}$; $\mathrm{B}=0.05 \mathrm{~mm} ; \mathrm{C}=0.05 \mathrm{~mm} ; \mathrm{D}=0.10 \mathrm{~mm}$. 


\section{Depositories}

Material examined for this study is deposited in the following institutions:
$\mathrm{ABSC}=$ Archbold Biological Station, Venus, Florida, USA
CASC $=$ California Academy of Sciences, San Francisco, California, USA
$\mathrm{CNCI}=$ Canadian National Collection, Ottawa, Ontario, Canada
DEBU $=$ School of Environmental Sciences, University of Guelph, Guelph, Ontario, Canada
FMNH $=$ Field Museum of Natural History, Chicago, Illinois, USA
INBC = Instituto Nacional de Biodiversidad, Santo Domingo de Heredia, Costa Rica; now part of the Museo Nacional de Costa Rica
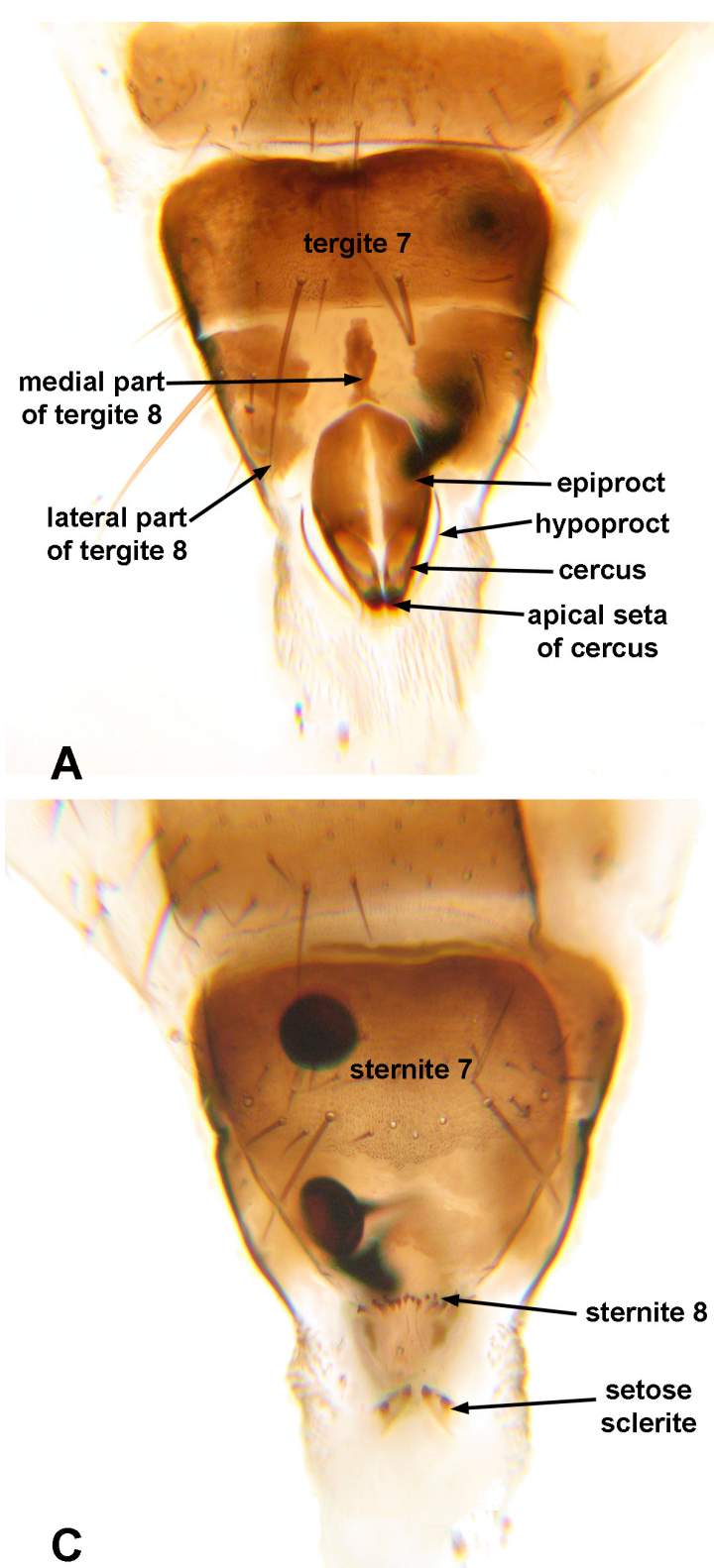
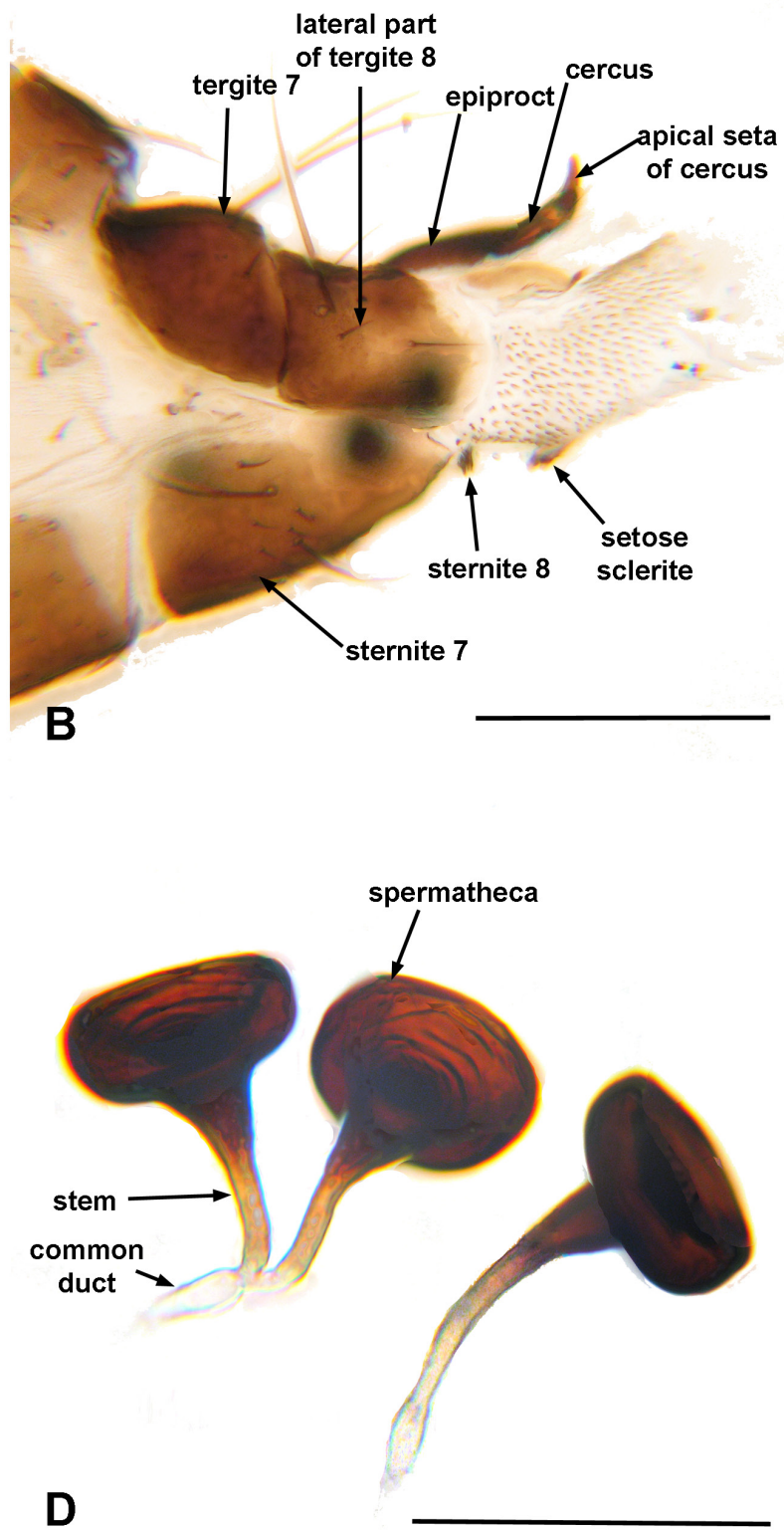

Fig. 4. Rudolfina megepandria sp. nov., female terminalia (debu01086086). A. Terminal abdominal segments, dorsal view. B. Same as preceding, lateral view. C. Same as preceding, ventral view. D. Spermathecae. Scale bars: A-C $=0.20 \mathrm{~mm} ; \mathrm{D}=0.05 \mathrm{~mm}$. 


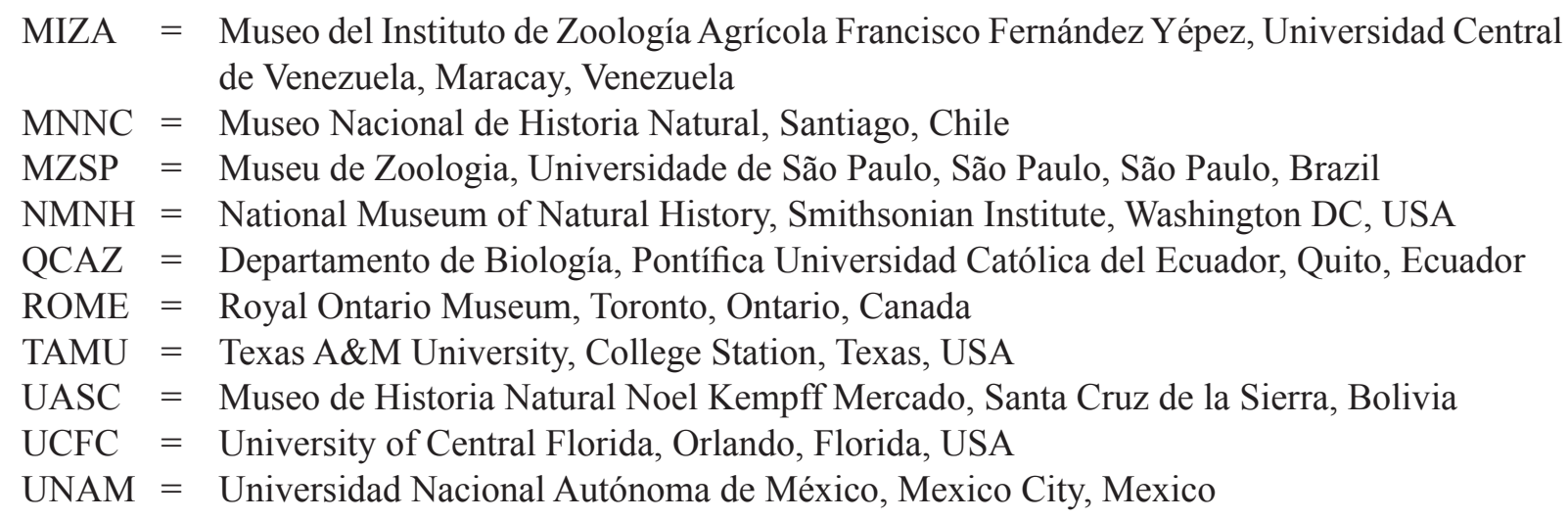

\section{Illustrations and photography}

Microphotographs of male and female genitalia were obtained using a Canon PowerShot S5IS mounted on a Leitz Laborlux 11 compound microscope. Series of images were aligned and combined using Zerene Stacker ver. 1.04 (Zerene Systems LLC, Richland, WA, USA) with the DMax algorithm. Additional editing with Adobe Photoshop CS5 (Adobe, San Jose, California, USA) was done to enhance clarity of the genitalic characters. All captions list the unique specimen identifier for the specimen(s) photographed.

\section{Phylogenetic analysis}

A character matrix (Table 1) was generated using Mesquite (ver. 3.10; Maddison \& Maddison 2017) and exported for analysis in TNT (Goloboff et al. 2008) using Traditional Search, with 10 random seeds and 5000 replications with the tree bisection re-connection (TBR) swapping algorithm. Trees were optimized in WINCLADA (Nixon 2002).

The following 36 morphological characters used in the phylogenetic analysis are organized by body region and sex. Character states were polarized using a hypothetical groundplan similar to $R$. cavernicola Marshall \& Fitzgerald, 1997 and generated based on character states in the outgroup genera Bitheca Marshall, 1987 and Bromeloecia Spuler, 1924. Rudolfina zhangi Su, 2017 was only recently described and no material was available for study. This species was therefore excluded from the phylogenetic analysis, although the description suggests that it is closely related to $R$. rozkosnyi Roháček, 1975, the only other Palaearctic species in the genus. Table 1 gives the character states for each species.

\section{Character states}

\section{Head}

1. Eye height:genal height ratio: (0) $1: 1$; (1) $1.5: 1$; (2) $2: 1$; (3) $2.5: 1$.

\section{Thorax}

2. Acrostichal setulae - number of rows anterior to suture: (0) 6 rows; (1) 8 rows.

\section{Wings}

3. Costagial seta length: (0) short, apex not surpassing humeral break; (1) long, apex surpassing humeral break but not reaching subcostal break; (2) very long, apex reaching or surpassing subcostal break.

\section{Legs}

4. Mid tibia (male) with apical ventral setal comb: (0) well developed; (1) reduced.

5. Mid femur (male) - ventral cluster of setae at base: (0) well developed, with 5 or more robust setae present; (1) reduced, with $<5$ robust setae present. 


\section{Male abdomen}

6. Sternite 5 - posterior lobes: (0) absent; (1) present.

7. Sternite 5 - medial process: (0) absent; (1) present.

8. Sternite 5 - depth of posteromedial emargination: (0) < 1/4 length of sclerite or more; (1) $1 / 3-1 / 4 ;(2)>$ $1 / 3$. The length (depth) of the emargination is treated here as a linear transformation series.

9. Sternite 5 - clusters of setae flanking posteromedial emargination: (0) absent; (1) present.

10. Epandrium - prominence: (0) regular, equal in width to tergite 5; (1) swollen, wider than tergite 5.

11. Epandrium - subanal plate: (0) incomplete; (1) complete.

12. Subepandrial sclerite: (0) transverse; (1) arched.

13. Cercus - development: (0) well developed, prominent; (1) reduced.

14. Cercus - general shape: (0) pad-like, weakly conical; (1) elongate conical.

15. Cercus - projecting posteriorly: (0) no; (1) yes.

16. Cercus - distal seta: (0) regular; (1) robust and enlarged.

17. Surstylus - posterior lobe: (0) rounded, not projecting; (1) elongate, extending posteriorly.

18. Surstylus -flattened/modified seta on posterior surface of posterior lobe: (0) absent; (1) present.

19. Surstylus - anterobasally distinctly rounded: (0) no; (1) yes.

20. Surstylus - anterior laminate lobe well-developed: (0) no, (1) yes.

21. Surstylus - anterior laminate lobe modified: (0) no; (1) weakly modified; (2) strongly modified.

22. Postgonite - shape at mid length: (0) parallel sided; (1) narrowed.

23. Postgonite - apical morphology: (0) simple; (1) with distinct apical setula.

24. Distiphallus - dorsal sclerite with distinct swellings along length: (0) absent; (1) present.

\section{Female abdomen}

25. Median part of tergite 8: (0) absent; (1) present.

26. Median part of tergite 8 - shape: (0) wider than long; (1) longer than wide.

27. Sternite 8: (0) well developed, evenly sclerotized; (1) weakly sclerotized.

28. Bisetose sclerites posterior to sternite 8: (0) absent; (1) present.

29. Epiproct: (0) entire; (1) medially desclerotized.

30. Epiproct - anterior margin: (0) rounded; (1) straight.

31. Epiproct: (0) anteriorly truncate or rounded; (1) anteriorly produced.

32. Cercus - shape: (0) ovoid; (1) strap-like.

33. Cercus - development: (0) well-developed; (1) reduced.

34. Cercus - dorsal chaetotaxy: (0) evenly hirsute; (1) glabrous.

35. Cercus - anterolaterally fused with epiproct: (0) no; (1) yes.

36. Cercus - apical seta: (0) unmodified; (1) flattened. 


\title{
Results
}

\section{Genus redescription, relationships, biology, distribution and phylogeny}

\author{
Class Insecta Linnaeus, 1758 \\ Order Diptera Linnaeus, 1758 \\ Superfamily Sphaeroceroidea Macquart, 1835 \\ Family Sphaeroceridae Macquart, 1835 \\ Subfamily Limosininae Macquart, 1835
}

Genus Rudolfina Roháček, 1987

Rudolfia Roháček, 1982: 225 (feminine, a junior homonym of Rudolfia Wilson, 1924 (Crustacea: Copepoda); type species: Limosina rozkosnyi Roháček, 1975, by monotypy).

Rudolfina Roháček, 1987: 474 (feminine, nom. nov. for Rudolfia Roháček, 1982; type species: Limosina rozkosnyi Roháček, 1975, automatic).

Rudolfia - Roháček 1982: 225 [formal diagnosis, phylogeny]; 1983: 152-154 [full description, phylogenetic notes]; 1987: 474 [homonymy]. — Marshall \& Richards 1987: 999-1000 [diagnosis in key, illustr.].

Rudolfina-Roháček 1998: 483 [diagnosis, illustration]. — Marshall \& Buck 2010: 1180-1183 [diagnosis in key, phylogenetic notes, biology]. — Su et al. 2017: 392-393 [key].

\section{Redescription}

BoDy. Colour light to dark brown. Length 1.4-2.3 mm.

HEAD. With 3-5 interfrontal setae (of equal length or the foremost shorter), 1 (rarely 2) inclinate orbital setula and 4-10 small orbital setulae inside and below 2 strong, exclinate orbital setae; ocellar triangle with pair of strong setae and 3-5 additional small setulae; outer vertical seta strong, exclinate; inner vertical seta inclinate; occipital and paravertical setae inclinate, well-developed; postocellar seta inclinate, weakly developed. Eye-to-gena height ratio variable between species (1.5:1 to 3.5:1). Vibrissa strong. Gena with 1-2 strong subvibrissal setae and 4-9 smaller setulae.

Thorax. Surface pruinose. Postpronotal lobe with 2-3 setae, outer seta strong, inner seta(e) reduced. Notopleural seta, 2 supra-alar setae and prescutellar dorsocentral seta strong. Acrostichal setulae in 4-8 rows, with 1 enlarged prescutellar acrostichal seta (almost as long as dorsocentral). Katepisternum with strong elongate posterior seta and reduced anterior seta.

Legs. Fore femur with 3-5 elongate setae dorsally (except $R$. exuberata sp. nov.). Fore tibia with 3-5 elongate setae ventrally. Mid femur with row of 3-10 anterodorsal setae extending from base, row of $2-5$ dorsal setae on apical $1 / 4$, and basal cluster of 4-21 small setae ventrally; males usually with additional ventral seta (often in ventrobasal cluster). Mid tibia with 4 dorsal setae (basal anterodorsal, medial anterodorsal, distal anterodorsal and distal posterodorsal); males with ventral comb of 4-13 setae on apical $1 / 2$ or less ( $R$. exuberata sp. nov. and $R$. remiforma sp. nov. with setae of ventral comb weakly developed); females usually with midventral seta (absent in $R$. megepandria sp. nov.). Hind tibia with small apical ventral spur.

WING. Always fully developed, with wing tip reaching or exceeding apex of abdomen. Costa extending to or just beyond end of $\mathrm{R}_{4+5}$, and with single costagial seta $>2.0 \times$ length of nearby setae. $\mathrm{R}_{4+5}$ slightly curved towards costa distally. Cell $\mathrm{dm}$ with short stub veins of $\mathrm{M}_{1}$ and $\mathrm{Cu}-\mathrm{A} 1$ extending beyond $\mathrm{dm}$-cu. Alula narrow, posterior margin straight. 
AвDOMEN. Sternites and tergites well sclerotized and setose (posterior and lateral margins more densely setose). Male sternite 4 usually simple (rarely densely setose medially).

Male ABDomen. Posterior margin of sternite 5 with lobe on each side of medial emargination (shape and size of emargination and lobes vary among species). Transverse (ventral) portion of sternite 6 narrow; straight or weakly arcuate. Ring sclerite (in the right membrane of segment 7, possibly derived from a spiracle) large and distinct. Epandrium setose, often with larger setae lateral to anal opening, and with right anteroventral corner drawn out into a finger-like process that extends to the hypandrium. Male cercus usually distinct, fused with the epandrium (reduced and obscured beneath the epandrium in a few species; e.g., R. pilosa sp. nov., R. remiforma sp. nov.). Hypandrium (Fig. 3B) Y-shaped with emarginate posteromedial extension; hypandrial arms posteriorly deeply bilobed posteriorly, with lateral lobe articulating with the epandrium and the medial lobe articulating with posterolateral corner of hypandrium. Pregonite distinct, small, near anterior base of postgonite. Postgonite generally simple and slender, with 3-4 setulae on anterior margin but modified in some species; ejaculatory apodeme small and finger-like, with small globular sperm pump, usually close to the basiphallus (easily lost during dissection); basiphallus simple (without an epiphallus); distiphallus with distinct elongate dorsal sclerite; acrophallus with dorsolateral lobes and a single ventral sac (often reduced).

Female ABDomen. Tergite 8 apparently tripartite, with two lateral triangular sclerites and a medial sclerite (reduced in several species). Epiproct bare except for usual pair of small setae and a few scattered setulae, strongly sclerotized, and fused laterally with cerci (except in $R$. cavernicola). Cercus with single flattened apical seta. Sternite 7 variable. Sternite 8 weak, transverse, covered in small setulae; pair of small, bispinose plates along posterior margin. Hypoproct very narrow, forming horseshoe-shaped band immediately below the cerci. Spermathecae (1 pair +1 single) generally disc-shaped or lenticular, with thin, long sclerotized ducts.

\section{Related and similar genera}

All species of Rudolfina will key out to "Rudolfia" in the key to Nearctic Sphaeroceridae by Marshall \& Richards (1987) but they will key out as "Archiceroptera genus complex, in part" at couplet 72 in the key of Marshall \& Buck (2010) to Neotropical Sphaeroceridae. This previous treatment reflected uncertainty about the limits between Rudolfina and the many undescribed Neotropical species in the Archiceroptera genus complex. The Archiceroptera complex is part of a larger group of Limosininae (including Aptilotella Duda, 1924, Archiceroptera Papp, 1977, Bitheca Marshall, 1987, Bromeoloecia Spuler, 1924, Pterogramma Spuler, 1924 and Robustagramma Marshall \& Cui, 2005) characterized by an unusual process extending medially from the lower right margin of the epandrium to the hypandrium. The relationships within this group need further study but the morphological analysis by Paiero (2017) suggests that Rudolfina is closely related to Bromeloecia. Within this group, Rudolfina resembles Archiceroptera in characters of the female epiproct and cercus. However, in Archiceroptera the epiproct is completely desclerotized medially (anteriorly sclerotized in Rudolfina), the cercus is separate from the epiproct and has a partially concave inner margin, and sternite 8 is divided into a pair of elongate lateral sclerites without the paired setulose sclerites found in Rudolfina. Archiceroptera species also differ from Rudolfina in having $\mathrm{M}_{1}$ extending as a pseudovein to the wing margin, $\mathrm{CuA}_{1}$ rarely with a distinct stub vein, the male cercus free from the epandrium and with a distinct ventral process, and (in many, but not all species) five or more dorsal mid tibial setae and two or more inclinate orbital setulae.

\section{Biology}

Roháček (1987) recorded R. rozkosnyi from dung and occasionally from mud and decaying vegetation, but most of the new species considered here were collected in dung or carrion traps. Larvae remain unknown. 


\section{Distribution}

Rudolfina has a mostly western Nearctic montane distribution, with high endemism in the southwest and the mountains of Mexico (Sierra Madre del Sur, Sierra Madre Oriental and Sierra Madre de Chiapas). Two widely separated species occur in the Palaearctic region ( $R$. rozkosnyi and $R$. zhangi) and one species ( $R$. exuberata sp. nov.) is widespread at low elevations from the southern United States to South America. Other than $R$. exuberata sp. nov., no true species of Rudolfina are known from south of Guatemala. Other Neotropical species previously treated as Rudolfina are discussed below.

\section{Results of the phylogenetic analysis}

Twenty-seven most parsimonious trees were generated, summarized here as a strict consensus tree (Fig. 5) and a majority rules consensus tree (Fig. 6). Characters were optimized on one of the equal length trees (Fig. 7) which was selected based on the recovery of several groups supported by putatively higher weight characters. Shared male genitalic morphology supported a close relationship of Rudolfina bucki sp nov. with $R$. megepandria sp. nov. and $R$. tumida sp. nov. with $[R$. rozkosnyi $+R$. digitata + $R$. zhangi]. The combined elongation of the epiproct and female cercus suggests that $R$. newtoni sp. nov. is closely related to $[R$. exuberata sp. nov. $+R$. pauca sp. nov. $+R$. remiforma sp. nov.], although the form of the epiproct in $R$. newtoni sp. nov. is apparently intermediate between the strongly elongated form found in the $R$. exuberata clade and the shorter epiproct of other Rudolfina. All trees recovered $R$. cavernicola as a sister taxon to the remaining species, which form a monophyletic group characterized by the fusion of the female cercus with the posterolateral corner of the epiproct, the elongation of the medial part of tergite 8 and by characters of the male cercus and surstylus. This tree suggests a New World origin for Rudolfina.

Within Rudolfina excluding $R$. cavernicola, $R$. rozkosnyi, $R$. digitata and $R$. tumida sp. nov. appear to be a basal grade predating the origin of a clade comprising the Mexican-Guatemalan species. These four

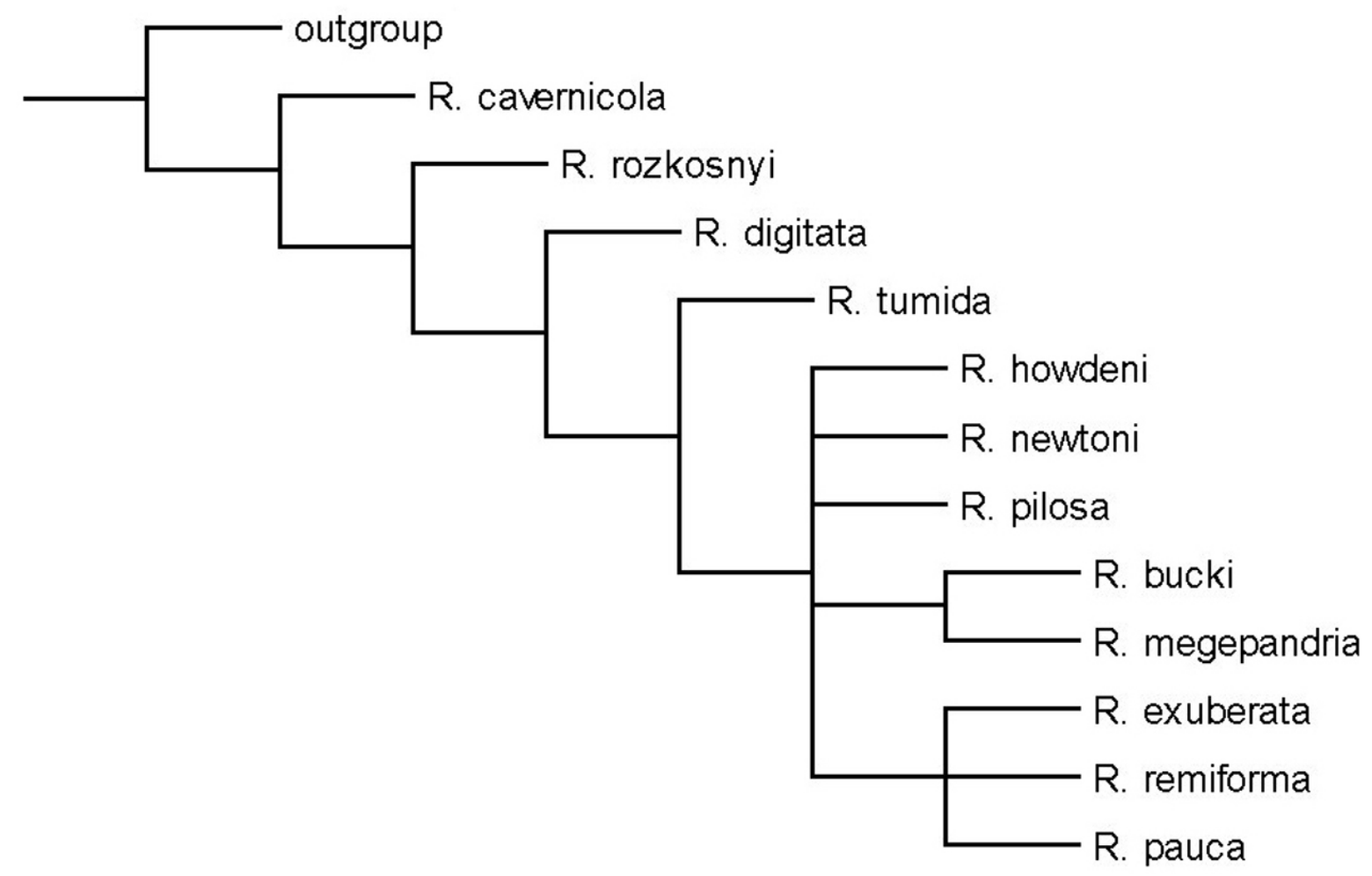

Fig. 5. Strict Consensus Tree for the 27 recovered trees obtained from Traditional Search (TNT). 
species all have a laminate lobe on the surstylus, apparently derived from the simple laminate margin of $R$. cavernicola (absent in other species). The largely Mexican-Guatemalan clade can be recognized by the simple, rounded anterior lobe of the male surstylus and the absence of dorsal swellings on the dorsal sclerite of the distiphallus. The $R$. exuberata group (including $R$. exuberata sp. nov., $R$. remiforma sp. nov. and $R$. pauca sp. nov.) is characterized by a small elongate male cercus, tulip-shaped epiproct, and reduction of the female cercus. Rudolfina remiforma sp. nov. and $R$. pauca sp. nov. are known from only a few localities at higher elevations, as is typical of the genus, but the widespread $R$. exuberata sp. nov. occurs at much lower elevations than its more localized congeners.

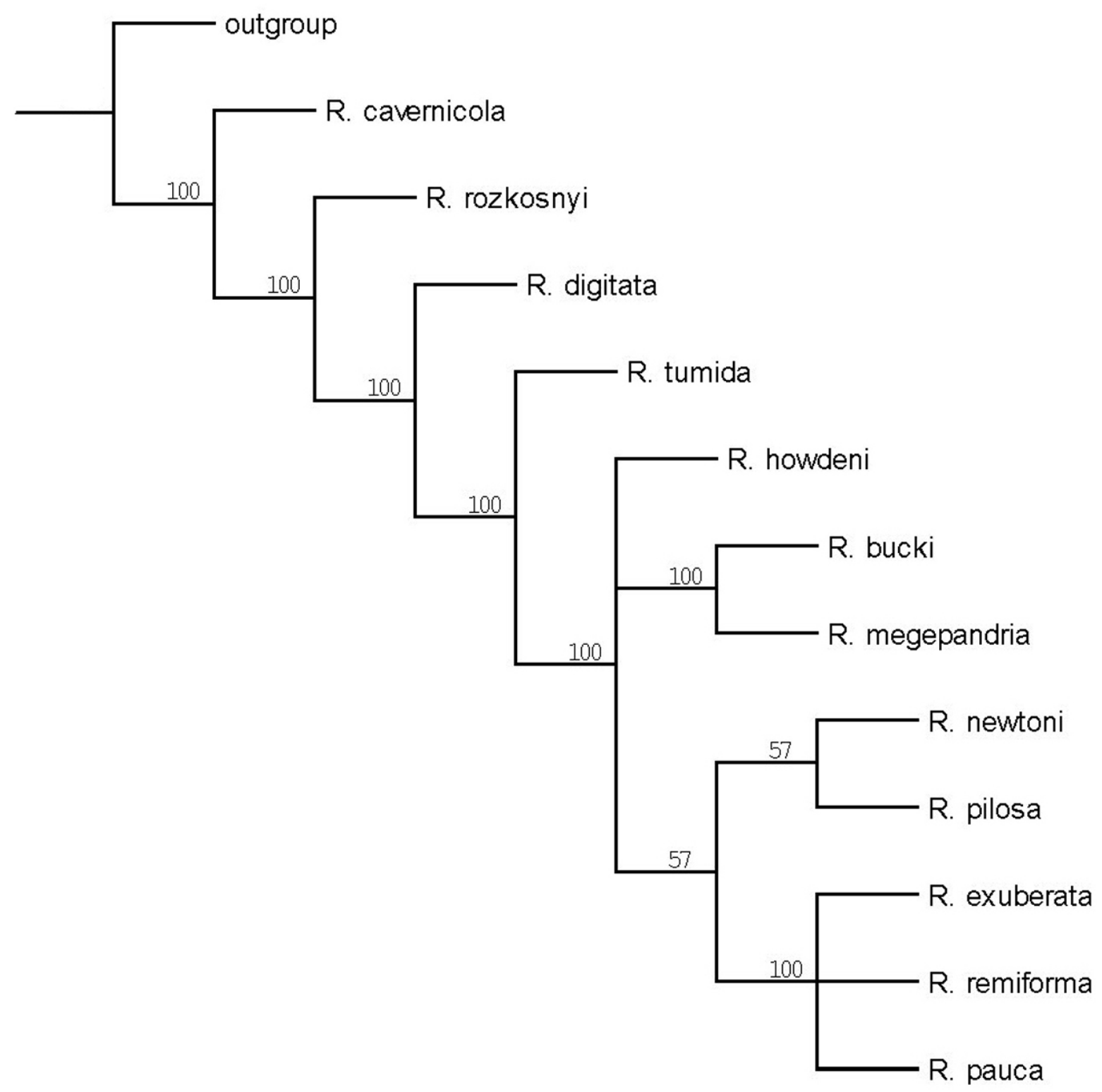

Fig. 6. Majority Rules Consensus Tree from the 27 recovered trees retained from Traditional Search (TNT). 


\section{Key to the New World Rudolfina}

Accurate identification of species of Rudolfina is largely dependent on examination of male sternite 5 and genitalic characters of both sexes; dissection may be required. Females of $R$. tumida sp. nov., $R$. bucki sp. nov., $R$. pilosa sp. nov. and $R$. zhangi are unknown.

1. Males

- Females

2. Sternite 5 with dense clusters of setae on each side of posteromedial emargination (Figs 14C, 18C)

- Sternite 5 evenly setose, without distinct clusters of setae

3. Eye height $\sim 2.5 \times$ genal height. Sternite 4 medially with cluster of long setae (denser along posterior margin, Fig. 18C). Sternite 5 with triangular lobe on each side of triangular medial emargination on posterior margin; emargination extending anteriorly $1 / 2$ length of sternite. Surstylus (in lateral view) boot-like, with 4-6 long setae originating from median knob on posterior surface; distal $1 / 3$ evenly covered in small setulae. Postgonite with distinct apical swelling

R. pilosa sp. nov.

- Eye height $\sim 1.5 \times$ genal height. Sternite 4 evenly setose (Fig. 14C). Sternite 5 with small nipple-like lobe on each side of medial emargination on posterior margin; emargination extending anteriorly almost to base of sternite. Surstylus (in lateral view) strap-like, elongate and narrow; relatively bare except for small scattered setae. Postgonite simple apically, uniformly narrow .

R. newtoni sp. nov.

4. Posterior margin of sternite 5 with elongate, parallel-sided lobes (e.g., Fig. 9C) on each side of medial emargination; pair of long setulae on margin of desclerotized area adjacent to base of the lobes. Length of $\mathrm{M}_{1}$ between crossveins dm-cu and $\mathrm{r}-\mathrm{m}<1.4 \times \mathrm{dm}-\mathrm{cu}$

- Posterior margin of sternite 5 with an acutely angled lobe on each side of medial emargination (e.g., Fig. 19C); emargination without long setulae. Length of $M_{1}$ between crossveins dm-cu and r-m usually $>1.5 \times \mathrm{dm}-\mathrm{cu}$

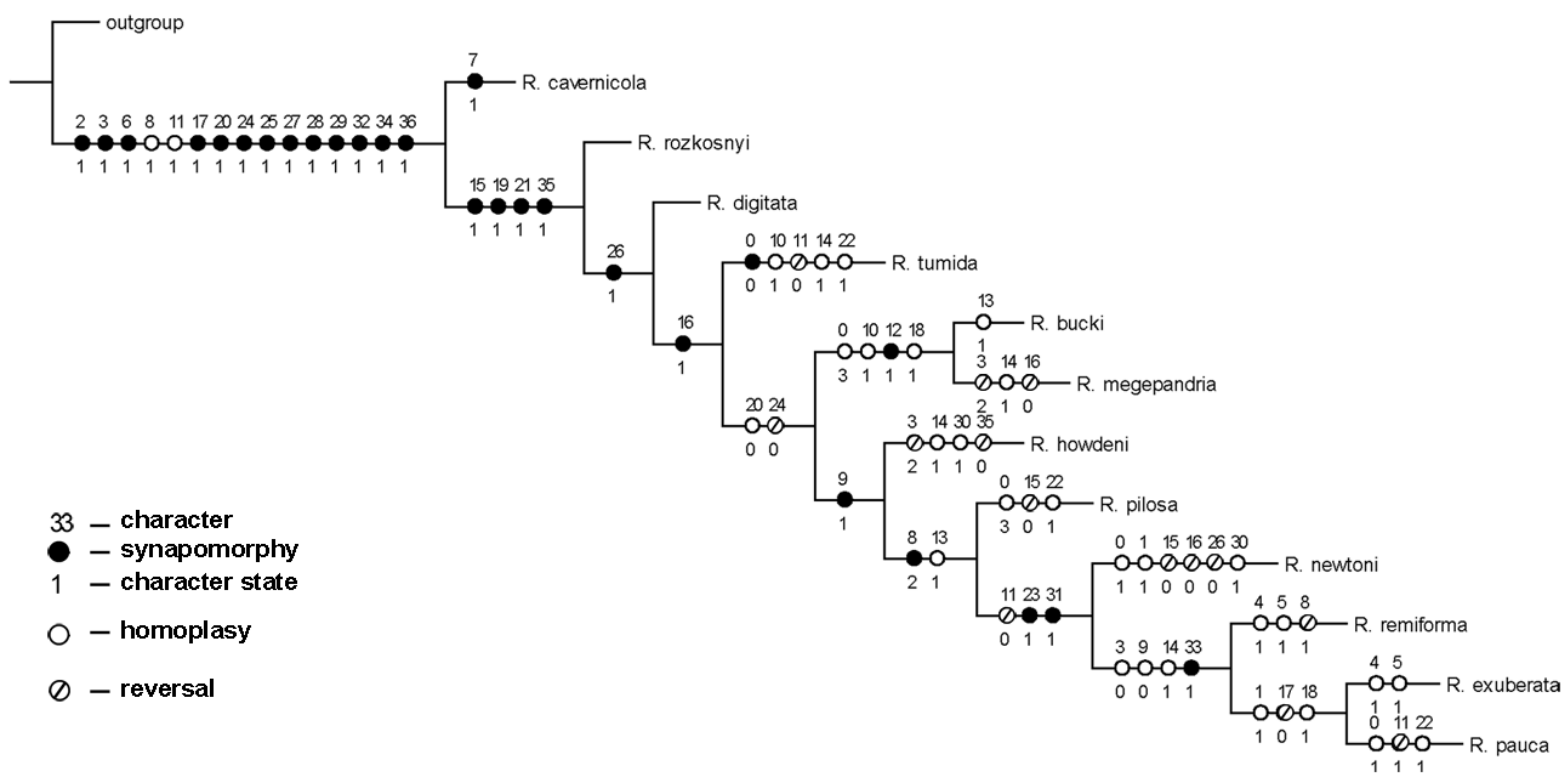

Fig. 7. Phylogeny of species of Rudolfina Roháček, 1987. Characters and character states refer to those given in Table 1. Tree selected from among 27 equal length trees. Length $=72, \mathrm{Ci}=56, \mathrm{Ri}=58$. 
5. Second costal sector $<0.4 \times$ third costal sector. Mid tibia with ventral comb of seta weakly developed, with only 1 strong seta at midlength and 1 long preapical seta. Mid femur with single distinct ventrobasal setae

R. exuberata sp. nov.

- Second costal sector $\sim 0.6 \times$ third costal sector. Mid tibia with ventral comb composed of 11-13 setae on apical $1 / 2$. Mid femur with $4-5$ small setae ventrobasally

R. pauca sp. nov.

6. Sternite 5 with small medial triangular lobe (Marshall \& Fitzgerald 1997: fig. 5). Surstylus strap-like, with base $\sim 1.5 \times$ as wide as distal margin; anterior margin weakly lamellate (Marshall \& Fitzgerald 1997: fig. 2)

R. cavernicola Marshall \& Fitzgerald, 1997

- Sternite 5 without medial lobe. Surstylus variable but usually with long posterior lobe and small anterior lobe

7. Sternite 5 with broad ( $>1 / 4$ width of sternite) emargination between posterior lobes. Subanal plate complete or incomplete

- Sternite 5 with small ( $<1 / 5$ width of sternite) emargination between posterior lobes. Subanal plate complete

8. Second costal sector $\sim 0.35 \times$ third costal sector. Subanal plate narrowly complete (Fig. 19A). Sternite 5 posterior margin lateral to posterior lobes straight (Fig. 19C). Cercus elongate, small. Surstylus with long, glabrous, oar-like posterior lobe and small rounded anterior lobe with 4-5 long setae on surface

R. remiforma sp. nov.

- Second costal sector $0.8-1.0 \times$ third costal sector. Subanal plate broadly complete or incomplete. Sternite 5 posterior margin lateral to posterior lobes emarginate (e.g., Fig. 3A-B). Cercus ovoid, large. Surstylus with posterior lobe variable; anterior lobe complex

9. Eye small, height $\sim 1.0 \times$ genal height. Length of $\mathrm{M}_{1}$ between crossveins $\mathrm{dm}-\mathrm{cu}$ and $\mathrm{r}-\mathrm{m} \sim 4.0 \times \mathrm{dm}-$ cu. Epandrium swollen, distinctly wider than two preceding abdominal segments (Fig. 2A). Subanal plate incomplete. Subepandrial sclerite distinctly arcuate .....

R. tumida sp. nov.

- Eye larger, height $>1.2 \times$ genal height. Length of $\mathrm{M}_{1}$ between crossveins dm-cu and $\mathrm{r}-\mathrm{m}<2.5 \times \mathrm{dm}$ cu. Epandrium unmodified, as wide as two preceding abdominal segments. Subanal plate broadly complete. Subepandrial sclerite transverse

10. Second costal sector equal to third costal sector. Surstylus with posterior lobe elongate, with irregular pectinate anterior surface (Marshall 1991: figs 4-5)

R. digitata Marshall, 1991

- Second costal sector $<1.0 \times$ third costal sector. Surstylus not as above, with posterior lobe either hoe-shaped or with 3 elongate processes .11

11. Eye height $2.0-2.3 \times$ genal height. Mid tibia distinctly arcuate in anterior view. Length of $M_{1}$ between crossveins dm-cu and $\mathrm{r}-\mathrm{m} \sim 1.5 \times \mathrm{dm}$-cu. Sternite 5 posterior margin with tips of medial lobes not reaching level of posterior margin adjacent to medial emargination (Roháček 1985: fig. 1084). Surstylus with posterior lobe hoe-shaped (Roháček 1985: fig. 1085)

R. rozkosnyi Roháček, 1975

- Eye height $\sim 1.3 \times$ genal height. Mid tibia weakly arcuate in anterior view. Length of $\mathrm{M}_{1}$ between crossveins $\mathrm{dm}$-cu and $\mathrm{r}-\mathrm{m} \sim 2.0 \times \mathrm{dm}$-cu. Sternite 5 posterior margin with tips of medial lobes extending beyond level of posterior margin adjacent to medial emargination ( $\mathrm{Su}$ et al. 2017: fig. 1F). Posterior lobe of surstylus with 3 elongate projections ( $\mathrm{Su}$ et al. 2017: fig. 1C)

R. zhangi $\mathrm{Su}, 2017$

12. Posteromedial emargination of sternite 5 with dark margin; emargination deep, extending anteriorly $\sim 1 / 6$ sternite length, and nearly closed posteriorly by inwardly directed lateral lobes (Fig. 11C). Epandrium simple, not distinctly wider than preceding abdominal segments. Surstylus with anterior lobe small but well-developed, knob-like; posterior lobe elongate, clavate, with numerous long setae on distal third. Cercus clavate, almost as long as surstylus, projecting ventrally.

R. howdeni sp. nov.

- Posterior emargination of sternite 5 without dark sclerotized margin; emargination shallow $(<1 / 10$ sternite length) and broadly open with short posteriorly projecting lateral lobes (Figs 8C, 13C). 
Epandrium swollen, distinctly wider than preceding abdominal segments. Surstylus with anterior lobe reduced and indistinct; posterior lobe either elongate and narrow ( $R$. megepandria sp. nov.) or weakly clavate ( $R$. bucki sp. nov.); setae more widely dispersed over apical $1 / 2$. Cercus either elongate and projecting posteriorly, or small and not distinctly projecting

13. Sternite 5 with small nipple-like lobes on posterior margin; posterior margin lateral to lobes entire (Fig. 13C). Epandrium (in lateral view) with dorsal surface as long as posterior surface; setae below anal opening typical, not elongated. Surstylus with posterior lobe narrow and elongate, weakly constricted on distal $1 / 4$, with small rounded swelling near midlength. Cercus elongate, projecting posteriorly. Postgonite apically acute

R. megepandria sp. nov.

- Sternite 5 with lobes on posterior margin triangular, obtuse; posterior margin lateral to lobes emarginate (Fig. 8C). Epandrium (in lateral view) with dorsal surface $\sim 1 / 2$ length of posterior surface; 4-6 pairs of long cruciate setae adjacent to cercus (usually obscuring cercus in caudal view). Surstylus with posterior lobe weakly clavate, with long thickened seta on posterior margin (near midlength). Cercus obscure, small, indistinct. Postgonite apically truncate ... $\boldsymbol{R}$. bucki sp. nov.

14. Epiproct tulip-shaped, with narrow anterior elongation broadening near midlength into rounded posterior 'bulb' (Figs 10A, 17A, 20A). Length of $\mathrm{M}_{1}$ between crossveins dm-cu and r-m $<1.5 \times$ dm-cu. Medial portion of tergite 8 small, weakly sclerotized. Cercus shorter than flattened apical seta.....

- Epiproct triangular or trapezoidal. Length of $\mathrm{M}_{1}$ between crossveins dm-cu and r-m $>1.5 \times \mathrm{dm}$-cu. Medial portion of tergite 8 distinct, well sclerotized. Cercus as long as or longer than flattened apical seta

15. Sternite 7 with posterior margin entire. Spermathecae ovoid (Fig. 20D)........R. remiforma sp. nov.

- Sternite 7 with posterior margin broadly emarginate (Fig. 10C). Spermatheca bilobed ................. 16

16. Eye height $2.0 \times$ genal height. Second costal sector $<0.5 \times$ third costal sector

- Eye height $2.5 \times$ genal height. Second costal sector $>0.5 \times$ third costal sector

R. exuberata sp. nov. R. pauca sp. nov.

17. Eye height $\sim 1.5 \times$ genal height. Medial part of tergite 8 posteromedially emarginate (Fig. 15A). Epiproct triangular. Spermathecae mushroom-shaped

R. newtoni sp. nov.

- Eye height $\geq 1.75 \times$ genal height. Medial part of tergite 8 posteriorly entire or desclerotized. Epiproct either trapezoidal or anteriorly rounded. Spermathecae variable 18

18. Eye height $2.5 \times$ genal height. Length of $M_{1}$ between crossveins dm-cu and r-m $3.0 \times \mathrm{dm}-\mathrm{cu}$. Medial part of tergite 8 elongate, rectangular (Fig. 4A)

R. megepandria sp. nov.

- Eye height $\leq 2.3 \times$ genal height. Length of $\mathrm{M}_{1}$ between crossveins dm-cu and r-m $2.0 \times \mathrm{dm}$-cu. Medial part of tergite 8 variable

19. Second costal sector shorter than third costal sector. Epiproct diamond-shaped, with anterolateral margins almost straight (Fig. 12A)

R. howdeni sp. nov.

- Second costal sector equal in length to third costal sector. Epiproct with anterolateral margins broadly rounded

20. Medial part of tergite 8 wider than long, with posterior margin weakly emarginate (Marshall \& Fitzgerald 1997: fig. 5). Epiproct triangular....

R. cavernicola Marshall \& Fitzgerald, 1997

- Medial part of tergite 8 as long as or longer than wide; posterior margin entire. Epiproct diamondshaped

21. Medial part of tergite 8 longer than wide (Marshall 1991: fig. 1). Epiproct with surface even, smooth; anterior margin broadly rounded

- Medial part of tergite 8 as long as wide (Roháček 1985: fig. 1079). Epiproct with surface wrinkled on posterior half; anterior margin weakly trilobed..... R. rozkosnyi Roháček, 1975 


\title{
Species descriptions (in alphabetical order)
}

\author{
Rudolfina bucki sp. nov. \\ urn:lsid:zoobank.org:act:2405E619-5A06-42BB-A033-00E43C70A5B6
}

Figs $8,21 \mathrm{~B}$

\section{Etymology}

The specific name is a patronym for Dr. Matthias Buck, a friend and previous sphaerocerid taxonomist.

\section{Material examined}

\section{Holotype}

MEXICO - 3; Oaxaca, 4.1 mi. W of Jct. Mex. and 175-Yuvila Rd; $9300 \mathrm{ft}$ a.s.1.; 8-19 Aug. 1973; A. Newton leg.; oak-fir-pine forest; FMNH debu01086238.

\section{Paratype}

MEXICO $\bullet 1$ ‡; same collection data as for holotype; DEBU.

\section{Description}

\section{Male}

Body. Length $1.6 \mathrm{~mm}$. Eye height $2.5 \times$ genal height. Head with 3-4 interfrontal setae and 4-5 small setae on inner margin of orbital plate. Gena with 2 strong setae and 7-8 smaller setulae. Acrostichal setulae in 4-6 rows. Costagial seta extending to midpoint between humeral and subcostal break. Second costal sector $0.7-0.8 \times$ third costal sector; length of $\mathrm{M}_{1}$ between crossveins dm-cu and r-m $3.5 \times \mathrm{dm}$-cu; $\mathrm{CuA}_{1}$ stub vein $\sim 3.0 \times \mathrm{M}_{1}$ stub vein. Male mid femur with 5-7 strong ventral setae and a circular cluster of 5-10 weaker setae ventrobasally. Male mid tibia with ventral comb of $4-5$ robust setae on apical $1 / 3$, without an enlarged midventral seta.

MaLe ABdomen (Fig. 8). Sternite 5 with pair of medial triangular lobes on posterior margin, distance between lobes $>1.0 \times$ basal width of single lobe, lobe length $\sim 1 / 5$ length of remainder of sternite. Transverse part of sternite 6 weakly arcuate. Epandrium strongly convex, with irregular cluster of 6-8 long, inclinate posterolateral setae (appearing fan-like in posterior view); subanal plate broad, complete. Surstylus with anterior lobe reduced to small basal swelling; posterior lobe elongate, clavate, weakly bent anteriorly on apical $1 / 3$; apical half of posterior lobe covered in small setae, with 1 robust elongate seta on posterior margin and 4-5 strong setae on inner surface. Cercus small, conical, with strong preapical seta. Postgonite apically truncate. Dorsal sclerite of distiphallus (Fig. 8E-G) without distinct swellings.

\section{Female}

Unknown.

\section{Remarks}

The male abdomen is similar to that of $R$. megepandria sp. nov., but males can be separated from that species by the shorter cercus and less pronounced epandrium.

Rudolfina cavernicola Marshall \& Fitzgerald, 1997

Fig. $21 \mathrm{~A}$

\section{Material examined}

The original type series from Kremmer's Cave was re-examined along with the following material.

MEXICO • 1 đ; San Luis Potosí, Cueva de Cinquenta y Ocho, 5 km S of San Francisco, $40 \mathrm{~km}$ E of San Luis Potosí, Municipio de Zaraqoza; 3000 m a.s.l.; 18 May 1972; Elliott, Ralph and Lynn leg.; DEBU. 


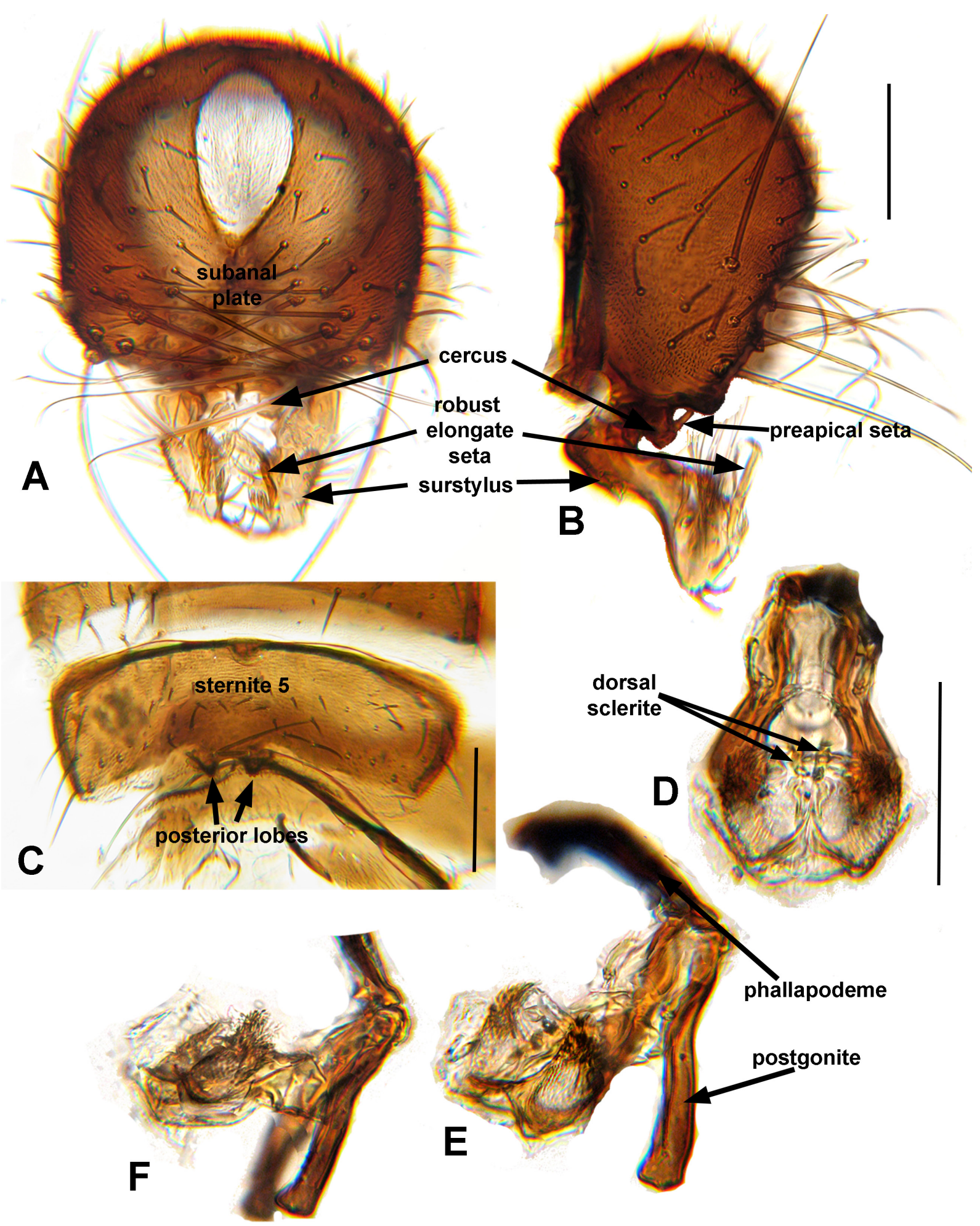

Fig. 8. Rudolfina bucki sp. nov., male terminalia (debu01086239). A. Epandrium, surstylus and cercus, caudal view. B. Same as preceding, lateral view. C. Sternite 5, ventral view. D. Phallus, dorsal view. E. Phallus, postgonite and phallapodeme, dorsolateral view. F. Same as preceding, lateral view. Scale bars: $\mathrm{A}-\mathrm{B}=0.10 \mathrm{~mm} ; \mathrm{C}=0.10 \mathrm{~mm} ; \mathrm{F}=0.10 \mathrm{~mm}$. 


\section{Description}

See Marshall and Fitzgerald 1997.

\section{Distribution}

Nearctic: United States of America (AZ, CO); Mexico (San Luis Potosí, newly recorded here).

\section{Remarks}

This species is newly recorded from Mexico. The illustration of this species that accompanied the original description (Marshall \& Fitzgerald 1997: fig. 1) includes what appears to be a large seta coming off the anterior base of the surstylus; this is an unsocketed laminate lobe.

\section{Rudolfina digitata Marshall, 1991}

Fig. 21A

\section{Material examined}

In addition to the original type material, the following material was examined:

CANADA - Alberta • 1 q; Coleman; 24-26 Jul. 1980; S.A. Marshall leg.; DEBU • 1 q; Hailstone Butte, $60 \mathrm{~km} \mathrm{~W}$ of Nanton; 21-23 Jul. 1987; S.A. Marshall leg.; dung cup, under cow parsnip; DEBU. British Columbia - 1 q; Kootenay Land District, Ainsworth, Woodbury Creek; 5 Jul. 1980; S.A. Marshall leg.; dung; DEBU • 1 đ̊; Okanagan-Similkameen, Mount Kobau; 1760 m a.s.l.; 29 May-3 Jun. 1991; Blades and Maier leg.; DEBU • 1 J゙; Peace River Land District, Pink Mountain; 16 Jul. 1987; S.A. Marshall leg.; marmot dung, dwarf willow; DEBU. - Ontario • 1 क; Agnes River; Jul. 1994; A.P. Applejohn leg.; SP2, plot 18; DEBU.

MEXICO - México • 2 + $\% ; 3$ mi. SW of Tenancingo, km 52¹/4; 2164 m a.s.1.; 31 Aug.-6 Sep. 1971; A. Newton leg.; oak-juniper, human dung; DEBU.

UNITED STATES OF AMERICA - New Hampshire • 1 đ; Coos Co., 1 mi. NE of East Inlet Dam; 25 Jun.-9 Jul. 1986; D.S. Chandler leg.; F.I.T.; DEBU. - New York • 1 đ̊; Greene Co., Cairo; 1 Jul. 1980; S.A. Marshall leg.; DEBU. - Wyoming • 2 ồ, 1 O; Sheridan Co., Antelope Butte Rec. Area, $20.3 \mathrm{~km}$ W of Burgess Jct.; 5-20 Aug. 1990; J.E. Swann leg.; along stream, pans, cow dung; DEBU • 1 ð;; Sheridan Co., Black Mountain, off Hwy 14; 5-20 Aug. 1990; J.E. Swann leg.; pine forest, pans, cow dung; DEBU • 2 ठิ ${ }^{\lambda}, 1$ +; Sheridan Co., Black Mountain, off Hwy 14; 5-20 Aug. 1990; J.E. Swann leg.; pines, lupines, pans; DEBU • 1 đ̊; Sheridan Co., Black Mountain, off Hwy 14; 5-20 Aug. 1990; J.E. Swann leg.; pines, pans, cow dung; DEBU.

\section{Description}

See Marshall (1991).

\section{Distribution}

Nearctic: Canada (AB, BC, YT, ON), U.S.A. (AK, CO, NH, NY, WY), Mexico (MEX).

\section{Remarks}

Rudolfina digitata, the second species to be described in this originally Palaearctic genus, is widely distributed in western North America and also occurs in a few eastern North American sites, including Mount Washington, New Hampshire. Mount Washington is known to have other species with disjunct western Cordilleran and eastern Appalachian distributions (e.g., Oeneis melissa (Fabricius, 1775) 
PAIERO S.M. \& MARSHALL S.A., A revision of the genus Rudolfina Roháček (Sphaeroceridae)

(Lepidoptera: Nymphalidae)), and the apparently disjunct population of $R$. digitata there suggests it may have once had a more extensive range. The other New Hampshire locality is also one of two known localities for the rare monotypic sphaerocerid genus Volumosina Roháček \& Marshall, 2017, otherwise known from one old growth forest in Ontario.

The male abdomen is most similar that of the Palaearctic R. zhangi.

Rudolfina exuberata sp. nov.

urn:1sid:zoobank.org:act:C4509588-8528-4024-A87C-67A845A8157E

Figs 1B, 9-10, 21D

\section{Etymology}

The specific name, which has been a manuscript name since Marshall (1982), is from the Latin for 'abundant': of the 1933 specimens examined for this study, 1649 were $R$. exuberata sp. nov.

\section{Material examined}

Holotype

UNITED STATES OF AMERICA • $\widehat{\jmath}$; Florida, Marion Co., Ocala National Forest; 14-18 Jun. 1984; S.A. Marshall leg.; creekside, dung; DEBU debu01087005.

\section{Paratypes}

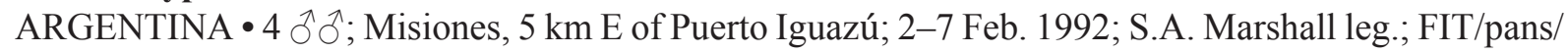
dung pans; DEBU.

BELIZE - Cayo • 4 ふึ ô, 2 q $q$; Caves Branch; 23-29 Aug. 1972; S. and J. Peck leg.: forest, dung; DEBU

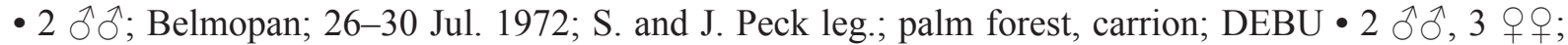
San Ignacio, Maya Mountain Lodge; $17^{\circ} 09^{\prime}$ N, 8904' W; 2 Jan. 1991; S.A. Marshall leg.; Atta mound trail; DEBU • 1 ô, 2 우; San Ignacio, Maya Mountain Lodge; $17^{\circ} 09^{\prime}$ N, 8904' W; 7-8 Jan. 1991; S.A. Marshall leg.; dung trap; DEBU 1 1 ${ }^{\top}$; San Ignacio, Maya Mountain Lodge; $17^{\circ} 9^{\prime} \mathrm{N}, 8^{\circ} 4^{\prime} \mathrm{W} ; 7$ Jan. 1991; S.A. Marshall leg.; Malaise; DEBU 1 ภ, 11 우; Mountain Pine Ridge, Hidden Valley Inst.; $2500 \mathrm{ft}$ a.s.l.; 10-15 Jan. 1991; S.A. Marshall leg.; broadleaf forest, dung traps; DEBU 1 ○; Mountain Pine Ridge, Hidden Valley Inst.; 2500 ft a.s.l.; 14 Jan. 1991; S.A. Marshall leg.; pine-jungle edge, sweep; DEBU • 1 J, 1 O ; Mountain Pine Ridge, Hidden Valley Inst.; $2500 \mathrm{ft}$ a.s.l.; 14 Jan. 1991; S.A.

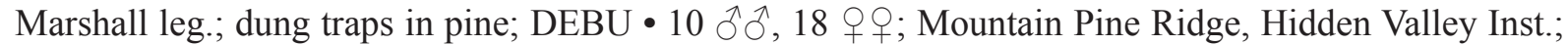
$2500 \mathrm{ft}$ a.s.1.; 10-15 Jan. 1991; S.A. Marshall leg.; grass-pine dung traps; DEBU • 3 $\partial^{\lambda}, 1$ $\circ$; Beaver Dam, 39 mi. W of Belize City; 6-10 Aug. 1972; S. and J. Peck; swamp forest, dung trap; CNCI.

BOLIVIA - La Paz $・ 1$ क; Heath River Wildlife Centre, $\sim 21 \mathrm{~km} \mathrm{SSW}$ of Puerto Heath; $12^{\circ} 40^{\prime} \mathrm{S}$,

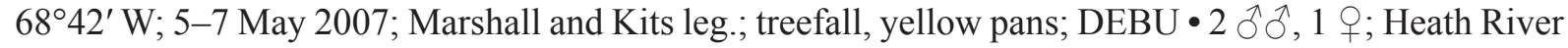
Wildlife Research Centre; $12^{\circ} 40^{\prime}$ S, 68 $42^{\prime}$ W; 5-9 May 2007; Paiero and Kits leg.; treefall, yellow

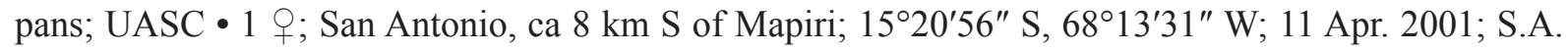
Marshall; secondary forest, dung pans; DEBU.

BRAZIL - Minas Gerais • $1 \hat{\partial}, 1$ क; 1 km E of Lavras; 18-20 Feb. 1990; S.A. Marshall leg.; dung traps in ditch; DEBU. - Paraná • 4 đิ đ̂, 2 우; Londrina; 1-2 Feb. 1990; S.A. Marshall leg.; carrion pan

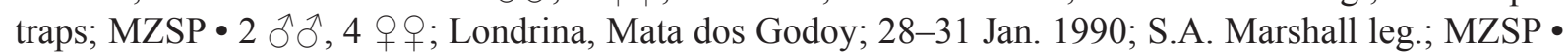
1 ग, 1 क; 30 km SE of Curitiba, BR 277; 6-9 Feb. 1990; S.A. Marshall leg.; dung traps; DEBU. - Rio

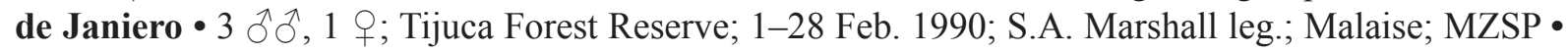
1 đa; Nova Friburgo, Sítio Edelweiss; 26 Jan. 1990; S.A. Marshall leg.; Malaise; DEBU. - São Paulo • 1 ک̊; Estación Biologica Boracea; 2 Dec. 2008; G.F.G. Miranda leg.; dung trap; MZSP・ 1 +; Sao Paulo, 
Jaragua; 8 Feb. 1990; S.A. Marshall leg.; DEBU • 8 ふぇ, 7 우; USP Biology Station; 5-6 Feb. 1979; R. Woodruff and J. Runnacles; human dung; MZSP.

CHILE • 2 q ; Valparaíso, La Campana National Park; 22 Nov. 2006; S.A. Marshall leg.; MNNC.

COLOMBIA $\bullet 2 \hat{\jmath} \hat{\jmath}, 2$ 우; $\mathrm{N}$ of Santander, $20 \mathrm{mi}$. S of Cucuta Quebrada de Honda; $2500 \mathrm{ft}$ a.s.l.;

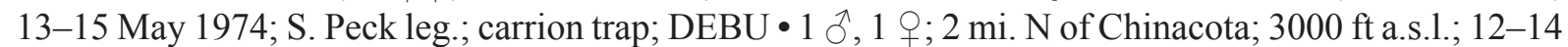
May 1974; S. Peck leg.; carrion; DEBU.

COSTA RICA - Alajuela • 1 q; Volcán Tenorio, N slope near Bijagua Biological Station; 700 m a.s.l.; 18 Jun. 2000; Buck and Marshall leg.; pan traps in tree fall; DEBU • 1 q; Río Peñas Blancas; $700 \mathrm{~m}$ a.s.1.; 18 Aug. 1986; L. Masner; DEBU 1 ô;; Florencia Forest; 28 Feb. 1980; H. Howden leg.; dung trap; DEBU. - Cartago $\bullet 7$ $\widehat{\jmath}, 7$ 우; Turrialba, CATIE; 600 m a.s.1.; 26 Feb. 1980; H. and A. Howden

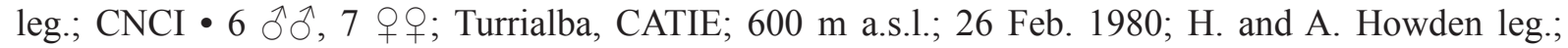
INBC • 6 ふぇ, 9 우; Turrialba, CATIE; 600 m a.s.1.; 28 Feb. 1980; H. and A. Howden leg.; DEBU • 1 ô; Turrialba, CATIE, Florence Forest; 600 m a.s.l.; 28 Feb. 1980; H. and A. Howden leg.; cup traps; DEBU. - Guanacaste 1 đं; Guanacaste Conservation Area, Pitilla Field Station; 29 Jan. 1996; J. Noyes leg.; Malaise; DEBU. - Heredia • 1 \%; La Selva; 2 Feb. 1990; N. Grieg leg.; around dung ball rolled by Canthon moniliatus Bates, 1887; DEBU. - Limón - 4 우; Estrella Valley, Pandora; 20 Feb. 1984; H. Howden leg.; carrion trap; CNCI 11 गे, 1 क; 4 km NE of Bribri; 50 m a.s.l.; Dec.1989-Mar. 1990; P. Hanson leg.; INBC.

CUBA • 1 q; Santiago, Gran Piedra Meteorological Radar; 1100 m a.s.1.; 6-17 Dec. 1995; S. Peck leg.; elfin forest, carrion traps; DEBU.

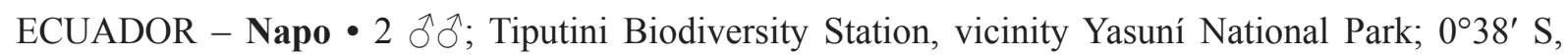
760' W; 14-19 Feb. 1998; D.C. Darling leg.; human dung pitfalls; DEBU • 1 q; Yasuní National Park, Yasuní Research Station; $0^{\circ} 38^{\prime}$ S, 76² 36 ' W; 3-20 Nov. 1998; Pape and Viklund leg.; rain forest, Malaise trap; DEBU $\bullet 4$ q ; J Jatun Sacha Reserve, $6 \mathrm{~km}$ E of Misahuallí; $1^{\circ} 04^{\prime} \mathrm{S}, 77^{\circ} 37^{\prime} \mathrm{W}$; $450 \mathrm{~m}$ a.s.1.; 30

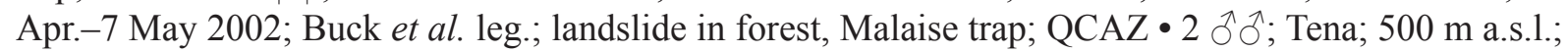

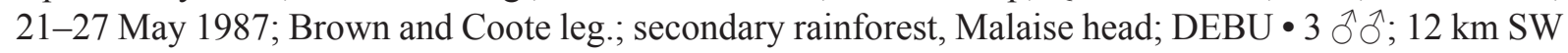
of Tena; 500 m a.s.1.; 8-11 Aug. 1976; S. Peck leg.; dung trap; QCAZ • 1 ô; S side of Rio Piocullin, SW Puerto Napo, S of Limonchicta; 600 m a.s.1.; 23-27 May 1987; primary lowland rainforest, Malaise

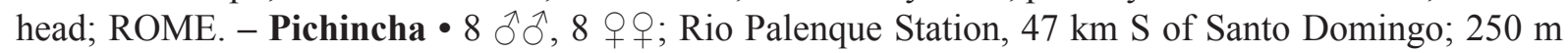

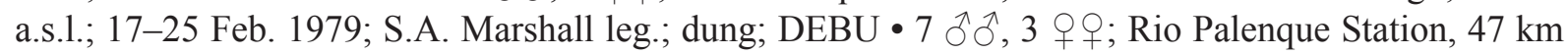
S of Santo Domingo; 250 m a.s.1.; 17-25 Feb. 1979; S.A. Marshall leg.; QCAZ • 1 đ̆; Rio Palenque; 25 Feb. 1979; S.A. Marshall leg.; carrion; QCAZ • 1 q; Rio Palenque; 27 Feb. 1979; S.A. Marshall

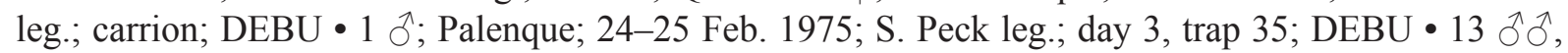
23 우; Rio Palenque; 25 Feb. 1979; S.A. Marshall leg.; dung; DEBU • $4 \hat{\partial} \widehat{\partial}, 17$ o 9 ; Rio Palenque; 27 Feb. 1979; S.A. Marshall leg.; dung; DEBU • 5 $\widehat{\partial} \widehat{\partial}, 17$ 우; Rio Palenque; 27 Feb. 1979; S.A. Marshall

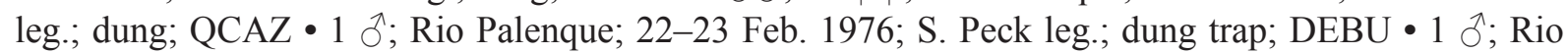
Palenque; 25-26 Feb. 1976; S. Peck leg.; dung trap; DEBU • 1 §; Rio Palenque; 22 Feb. 1976; S. Peck leg.; DEBU.

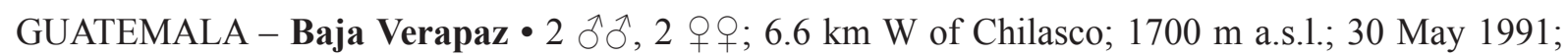
H. Howden leg.; dung; DEBU. - Guatemala • $1 \hat{\partial}, 1$; Santa Catarina Pinula; 1840 m a.s.l.; $11-13$ Jun. 1991; B.D. Gill leg.; dung traps; DEBU. - Petén • 11 đิ ô, 7 q $q$; Tikal; 28-30 Jul. 1978; Helava and Kukal leg.; dung trap; DEBU. - Sacatepéquez • 1 क ; $5 \mathrm{~km}$ SE of Antigua; 14³2'14" N, 90 41'41" W; 2125 m a.s.1.; 10-13 Jun. 2009; hardwood forest, Malaise trap; DEBU. - Zacapa • 1 §; $3.5 \mathrm{~km} \mathrm{SE}$ of La Unión; 1500 m a.s.l.; 26 Jun. 1993; R. Anderson leg.; cloud forest litter; DEBU • 3 ㅇ 0 ; same collection data as for preceding; 25-27 Jun. 1993; Ashe and Brooks leg.; FIT \#128; DEBU. 


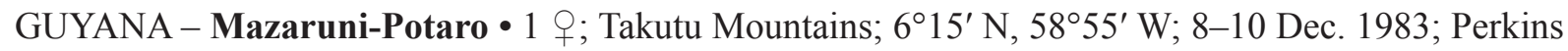
and Steiner leg.; window trap in montane rainforest near logging area; DEBU. - Potaro-Siparuni •

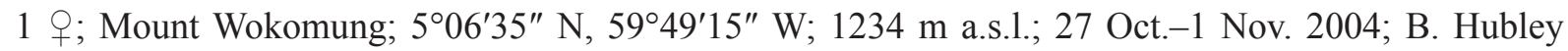
leg.; primary rainforest, human dung, pitfall trap; ROME. - Rupununi $\bullet 3 \hat{\delta} \delta^{2}, 4$ + $q$; Kurupukari, Essequibo River; $200 \mathrm{ft}$ a.s.1.; 9 Oct. 1990; B. Hubley leg.; primary forest, dung traps; ROME 1 ठ 1 क; Kabocalli, Iwokrama Forest Res.; 60 m a.s.1.; 3-5 Jun. 2001; Brooks and Falin leg.; FIT; DEBU.

HONDURAS • 1 \%; Olancho; 23 May 1995; R. Cordire leg.; FIT; DEBU.

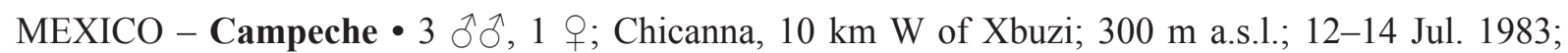

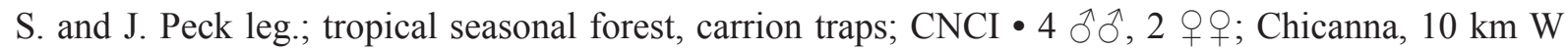
of Xbuzi; 300 m a.s.l.; 12-14 Jul. 1983; S. and J. Peck leg.; tropical seasonal forest, carrion traps; UNAM • $3 \widehat{\jmath} \widehat{\jmath}, 7$ q ; 53 mi. E of Escárcega; 500 ft a.s.1.; 8-14 Aug. 1971; A. Newton leg.; tropical semi-evergreen, dung; DEBU $\bullet 3 \hat{\partial} \hat{\partial}, 7$ q $O$; same collection data as for preceding; FMNH $\bullet 7 \hat{\delta} \hat{\partial}$, 9 우; 87 mi. E of Escárcega; $800 \mathrm{ft}$ a.s.1.; 8-14 Aug. 1971; A. Newton leg.; semi-evergreen, human

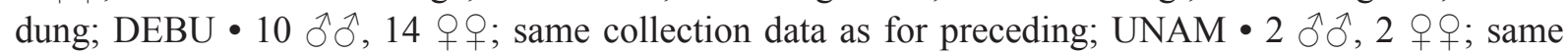
collection data as for preceding; FMNH. - Chiapas -2 ㅇ; 21 mi. N of Bochil; $5500 \mathrm{ft}$ a.s.1.; 18-24 Aug. 1971; A. Newton leg.; pine, oak, Liquidambar L., human dung; DEBU 1 9 ; 4 km SE of Custepec; $15^{\circ} 42^{\prime} 30^{\prime \prime} \mathrm{N}, 92^{\circ} 55^{\prime} 51^{\prime \prime} \mathrm{W} ; 2100$ m a.s.1.; 20 May 2008; cloud forest, Malaise trap; DEBU • 2 우우; Playón de la Gloria; $16^{\circ} 08^{\prime} 53^{\prime \prime}$ N, 90 $90^{\circ} 3^{\prime} 48^{\prime \prime}$ W; 180 m a.s.l.; 25 May 2008; mature wet forest, Malaise trap; UNAM • 1 q; Playón de la Gloria; 1609'37" N, 9054'7" W; 160 m a.s.1.; 25 May-24 Jun. 2008; mature wet forest, Malaise trap; UNAM • $2 \widehat{\delta} \delta^{2}, 4$ + $9 ; 8 \mathrm{~km}$ SE of Salto de Agua; $17^{\circ} 30^{\prime} 45^{\prime \prime} \mathrm{N}$, $92^{\circ} 17^{\prime} 40^{\prime \prime} \mathrm{W}$; 60 m a.s.1.; 14-17 Jun. 2008; secondary wet forest, Malaise trap; UNAM $\bullet 2$ क $\circ ; 8 \mathrm{~km} \mathrm{SE}$ of Salto de Agua; $17^{\circ} 30^{\prime} 58^{\prime \prime}$ N, 92 $2^{\circ} 18^{\prime} 05^{\prime \prime} \mathrm{W}$; $100 \mathrm{~m}$ a.s.1.; 14 Jun. 2008; wet forest edge, Malaise trap;

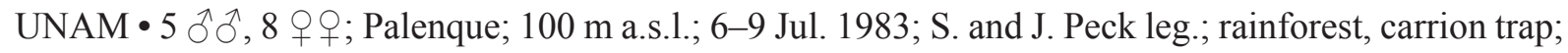
DEBU • 1 क; Parque Nacional Sumidero; 1000 m a.s.l.; 1 Jun. 1990; H. and A. Howden leg.; CNCI • 1 ô, 2 우; 15 mi. NW of Ocozocoautla; $2800 \mathrm{ft}$ a.s.l.; A. Newton leg.; rainforest, dung; DEBU • $5 \hat{\jmath} \widehat{\partial}$, 2 우; 11 mi. NW of Ocozocoautla; $3400 \mathrm{ft}$ a.s.1.; 19-25 Aug. 1971; A. Newton leg.; oak-evergreen

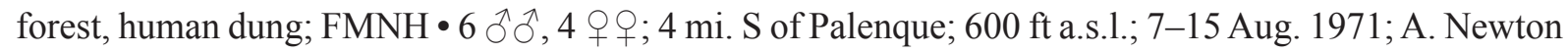
leg.; rainforest, human dung; DEBU $\bullet 10 \widehat{\partial} \widehat{\partial}, 10$ + $\odot$; same collection data as for preceding but $700 \mathrm{ft}$ a.s.l.; DEBU $\bullet 11 \widehat{\partial} \hat{\partial}, 10$ 우; same collection data as for preceding; FMNH $\bullet 1 \hat{\jmath}$; Palenque; $80 \mathrm{~m}$ a.s.1.; 2-23 Jul. 1983; S. and J. Peck leg.; secondary vegetation; FIT; DEBU • 1 đ; Laguna Belgica, 16 km NW of Ocozocoautla; 970 m a.s.l.; 13 Jun. 1990; H. and A. Howden and B. Gill leg.; F.I.T.; DEBU. - Guerrero • 1 ô, 1 q; 9 mi. NE of Iguala; 4400 ft a.s.1.; 29 Aug.-4 Sep. 1971; A. Newton leg.;

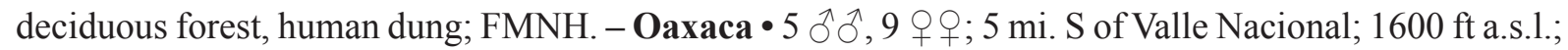

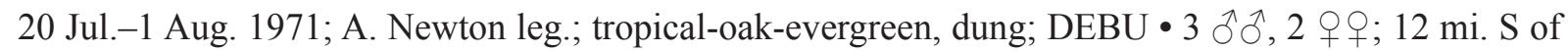
Valle Nacional; $3200 \mathrm{ft}$ a.s.1.; 22-31 Jul. 1971; A. Newton leg.; tropical montane forest, shrimp carrion; DEBU. - Pueblo • 1 Ō; 1 mi. S of Honey; 6800 ft a.s.1.; 1-6 Aug. 1971; A. Newton leg.; Pinus L.,

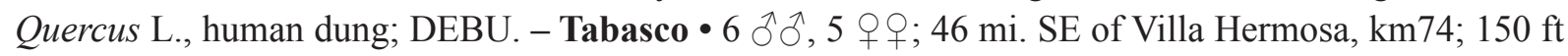
a.s.1.; 8-15 Aug. 1971; A. Newton leg.; secondary veg. rainforest, human dung; FMNH. - Tamaulipas

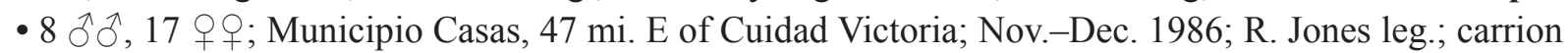
pitfall trap; TAMU. - Veracruz $\bullet 1$ ð’; $33 \mathrm{~km}$ NE of Catemaco, Los Tuxtlas Biological Station; $160 \mathrm{~m}$ a.s.l.; 6 Jul.-1 Aug. 1983; S. Peck leg.; ridge rainforest, FIT; DEBU $\bullet 2 \hat{\jmath} \widehat{\jmath}, 4$ 우; Fortin, SW of Rio Metlas; $3250 \mathrm{ft}$ a.s.l.; 13-18 Jul. 1971; A. Newton leg.; human dung, FMNH $\bullet 3$ $\partial^{\lambda}, 2$ $q$ + $; 10 \mathrm{mi}$. SW

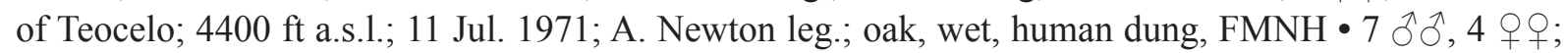
8 mi. NNW of Sontecomapan; Jul.-Aug. 1971; A. Newton leg.; rainforest, dung; DEBU. - Yucatán • 1 ; 9 mi. SE of Santo Roso, km 1371/4; 100 ft a.s.l.; Aug. 1971; A. Newton leg.; tropical sub-deciduous forest, human dung; DEBU. 


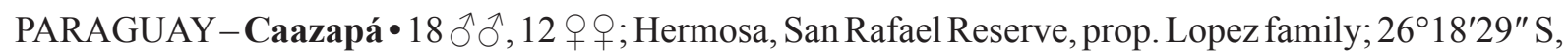

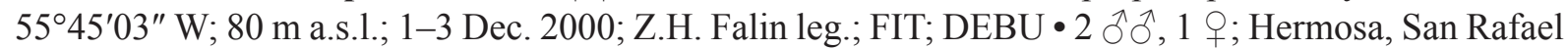

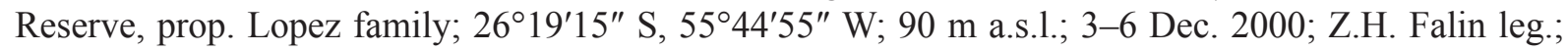

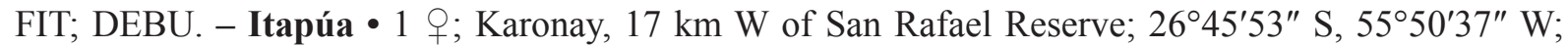
90-110 m a.s.1.; 18-21 Nov. 2000; Z.H. Falin leg.; FIT; DEBU.

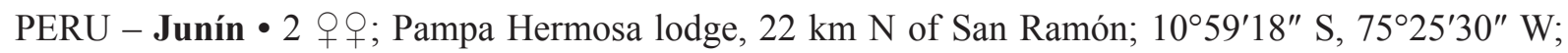
1220 m a.s.1.; 24-27 Nov. 2007; D. Brzoska leg.; FIT; DEBU. - Cusco • 1 + ; Cock-of-the-Rock Lodge,

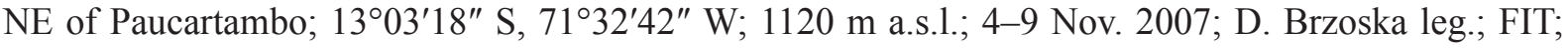
DEBU. - Lima - 1 ; Huaral, Chancay; 15 Mar. 1951; Ross and Michelbacher leg.; river valley; CASC.

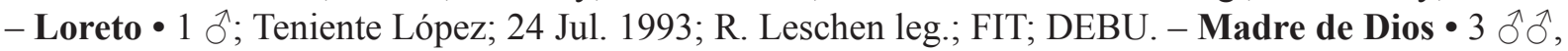

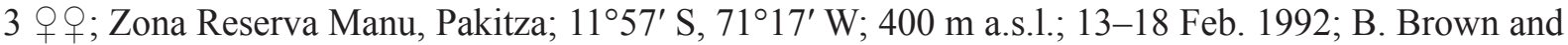
D. Feener leg.; Malaise trap; DEBU • 3 우; Pantiacolla Lodge, Alto Madre de Dios River; $12^{\circ} 39^{\prime} 18^{\prime \prime}$ S,

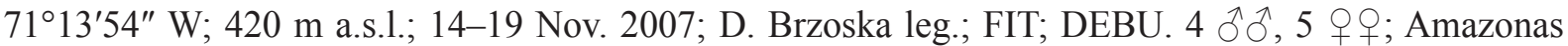
Lodge, $\mathrm{N}$ of Atalaya; $12^{\circ} 52^{\prime} 12^{\prime \prime} \mathrm{S}, 71^{\circ} 22^{\prime} 36^{\prime \prime} \mathrm{W} ; 480 \mathrm{~m}$ a.s.1.; 10-13 Nov. 2007; D. Brzoska leg.; FIT; DEBU • 1 क; Los Amigos Biological Station; 3-13 Jun. 2006; Paiero and Klymko leg.; Malaise; DEBU.

TRINIDAD AND TOBAGO - Tobago • 1 ते; $10 \mathrm{~km}$ NE of Roxborough, Gilpin Trail; $450 \mathrm{~m}$ a.s.1.; 26-30 Jun. 1993; S. and J. Peck leg.; rainforest, carrion traps; DEBU. - Trinidad • 1 ठ; University of Santa Cruz, Gasparillo; 15 Nov. 1987; R. Borneo leg.; grassland/forest edge, Malaise trap; DEBU.

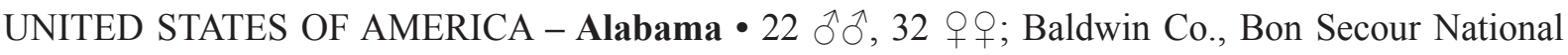
Wildlife Refuge; $30^{\circ} 14^{\prime} 48^{\prime \prime}$ N, 87 $49^{\prime} 45^{\prime \prime}$ W; 5-7 Jun. 1994; S.A. Marshall leg.; dung traps in oak; DEBU $\bullet 2$ 우; same collection data as for preceding; 5-7 May 1994; S.A. Marshall leg.; mushroom trap on oak; DEBU • 2 ふふ; Jackson Co., Paint Rock; 7-13 Jul. 1973; S. Peck leg.; dung; DEBU. - Arkansas - 1 đ̊; Johnson Co., Haw Creek Falls; 22 May 1991; B.J. Sinclair leg.; DEBU • 1 ô, 2 우 o; Logan Co., Ozark National Forest, Magazine Mountain; 23 May 1991; J.E. Swann leg.; mushroom baited pans; DEBU • 2 우; Madison Co., 3 mi. S of Brashers; 1600 ft a.s.l.; 19 Jun.-12 Jul. 1972; A. Newton leg.; hardwood forest, dung; DEBU • 1 o; Polk Co., Rich Mountain, 13 mi. NW of Mena; $2800 \mathrm{ft}$ a.s.1.; 1-3 Jun. 1979; S. and J. Peck leg.; mesic oak-hickory; DEBU $\bullet 4$ đô, 3 qo ; Washington Co., 3 mi. S of

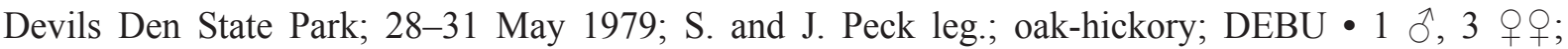
Washington Co., Devils Den State Park, Devils Den Cave; 28-31 May 1979; S. and J. Peck leg.; DEBU. - Florida • 1 of; Alachua Co., Gainesville; 20-30 Nov. 1986; W.R.M. Mason leg.; hardwood forest, Malaise trap; DEBU • 1 q; Alachua Co., Rd 176; 10 May 1983; K.W. Vick leg.; carrion trap; DEBU • 1 q; Alachua Co., Gainesville, DPI; 25-27 May 1983; D.S. Chandler leg.; Malaise trap; DEBU • 1 क ; Alachua Co., High Springs; 15-16 Dec. 1999; S.A. Marshall leg.; on dung; DEBU • 1 O; Alachua Co.,

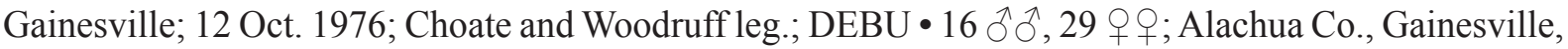
Hogtown Creek; 12 Oct. 1976; Choate and Woodruff leg.; DEBU $\bullet 2$ $\widehat{\jmath}, 5$ o $q$; Clay Co., Gold Head

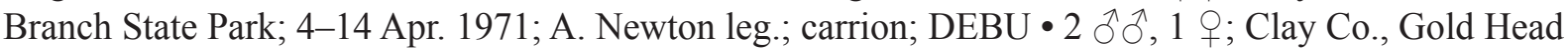
Branch State Park; 14 Apr. 1971; A. Newton leg.; dung; DEBU • 1 क; Collier Co.; 28 Dec. 1979; S.A. Marshall leg.; DEBU • 3 우; Collier Co., Collier Seminole State Park; Apr.1971; A. Newton; pine, palm, human dung; UCFC • 1 §, 6 우; Dade Co., Everglades National Park, $1.5 \mathrm{~km}$ NW of Royal Palm; 1 Nov. 1984-3 Mar.1985; S. and J. Peck leg.; hardwood hammock forest, Malaise-FIT; UCFC •

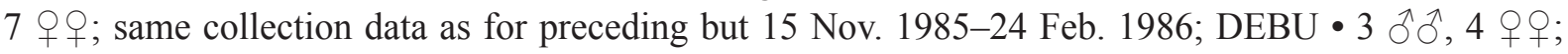
same collection data as for preceding but 2 May-2 Aug. 1985; DEBU • 5 q + ; Dade Co., Chekika State Recreation Area, 50 km SW of Miami, Grossman Hammock Forest; 1 May-2 Aug. 1985; S. and J. Peck leg.; Malaise-FIT; DEBU • 1 क; De Land Co., Hwy. 40, near Barberville; $18-20$ Jun. 1984; S.A. Marshall leg.; mushroom trap; DEBU • 1 đo, 1 क; Hendry Co., La Belle, Captain Hendry Rd; Apr.1971; pine, hardwood near river, human dung; A. Newton leg.; DEBU $\bullet 1$ ग, 3 q $ᄋ$; Hernando Co., Withlacoochee State Forest, 1 mi. W of Croom; Apr.1971, A. Newton leg.; pine and hardwood, human dung; DEBU • 
PAIERO S.M. \& MARSHALL S.A., A revision of the genus Rudolfina Roháček (Sphaeroceridae)

1 §ै; Hernando Co., 1 mi. W of Croom; Apr. 1971; A. Newton leg.; mixed forest; DEBU • 1 क ; Highlands Co., Archbold Biological Station; 12-18 Dec. 1985; S.A. Marshall leg.; mushroom bait on sand; ABSC

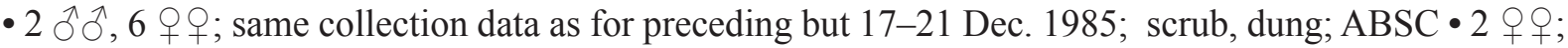
same collection data as for preceding but 12-16 Dec. 1985; scrub, dung pitfall; DEBU $\bullet 1$ के; same collection data as for preceding; scrub-sand, dung pan; DEBU $\bullet 6 \hat{\jmath}, 12$ 우; same collection data as for preceding but 15-19 Dec. 1985; dung pan; DEBU 1 o, 1 q; Highlands Co., Lake Placid, north

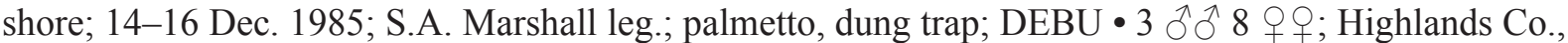
Highlands Hammock State Park, Cypress Swamp; 15-21 Dec. 1985; S.A. Marshall leg.; dung pan; DEBU 1 ô; Highlands Co., Highland Hammock State Park; 13-17 Apr. 1989; S.A. Marshall leg.; pans/maggot bait; DEBU • 1 đ, 6 우; same collection data as for preceding but 14-18 Jun. 1982; Woodruff and Rench leg.; orange grove, pig dung; UCFC • 30 $\widehat{\jmath}, 41$ 우; Lake Co., $1 \mathrm{mi}$. W of

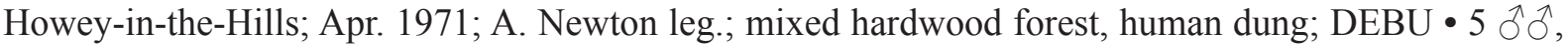
5 우; Lake Co., Howey-in-the-Hills; Apr. 1971; A. Newton leg.; dung; CASC • 5 ồ , 5 우; same collection data as for preceding; $\mathrm{CNCI} \bullet 5 \hat{\jmath}, 5$ 우; same collection data as for preceding; NMNH • 1 गे, 8 क 9 ; Liberty Co., Torreya State Park; 6 Jun. 1982; R.E. Woodruff leg.; pig dung trap; DEBU •

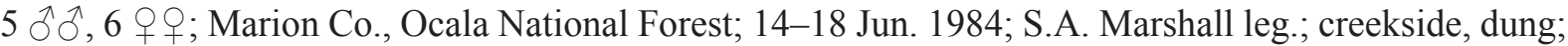
$\mathrm{DEBU} \bullet 10 \hat{\partial} \widehat{\partial}, 10$ q $Q$; s same collection data as for preceding; FMNH・10 $\hat{\partial}, 10$ 우; same collection data as for preceding; $\mathrm{CNCI} \bullet 3 \hat{\jmath}, 6$ 우; same collection data as for preceding but 11-15 Jun. 1984; hardwood swamp, dung trap; DEBU • $2 \widehat{\jmath}, 2$ + $\odot$; Marion Co., Ocala National Forest; 8-11 Jun. 1984;

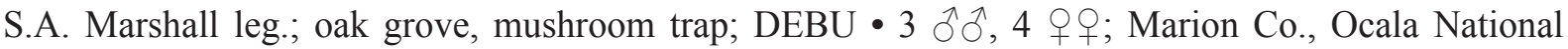

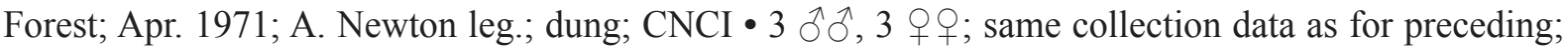

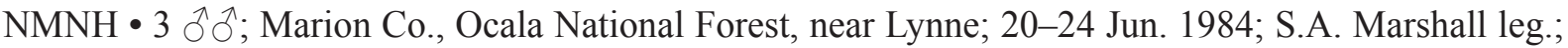

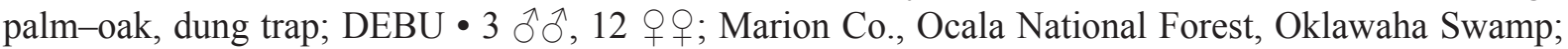
11-12 Jun. 1984; S.A. Marshall leg.; dung trap; DEBU • 2 q ; Marion Co., Ocala National Forest, Road 65; 18-20 Jun. 1984; S.A. Marshall leg.; sand-pine, human dung; DEBU • 1 §, 6 우; Marion Co., Ocala National Forest, Road 65, 1.5 mi. W of State Road 19; 15-16 Mar. 1984; R.E. Woodruff leg.; dung; DEBU • 1 đ̊; Marion Co., Ocala National Forest, Silver Springs Woods; 6-23 Jun. 1984; S.A. Marshall leg.; oak, FIT; DEBU • 1 q ; Marion Co., Ocala National Forest, Silver Springs Woods; $12-14$

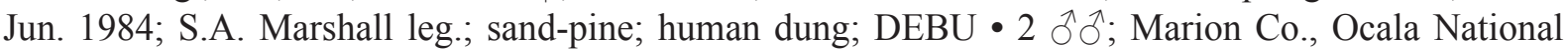
Forest, Silver Springs Woods; 11-15 Jun. 1984; S.A. Marshall leg.; carrion FIT; DEBU • 1 ठ, 1 ๆ; same collection data as for preceding but 6-22 Jun. 1984; FIT; DEBU • 2 क 9 ; same collection data as for preceding but 10-15 Jun. 1984; DEBU • 1 J; Marion Co., Ocala National Forest, Zay Prairie; 14-18

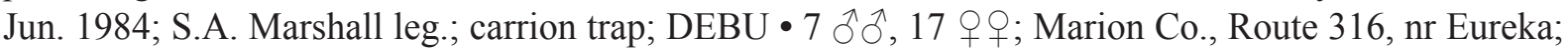
15-16 Mar. 1984; R.E. Woodruff leg.; pig dung trap; DEBU • 1 §’; Monroe Co., Everglades National Park, Royal Palm Hammock; 1 Nov. 1984-3 Mar. 1985; S. and J. Peck leg.; hammock forest, MalaiseFIT; DEBU • 6 ठ̊ $\widehat{\jmath}, 10$ 우; Nassau Co., golf course nr Amelia; 3-15 Apr. 1971; A. Newton leg.; oak

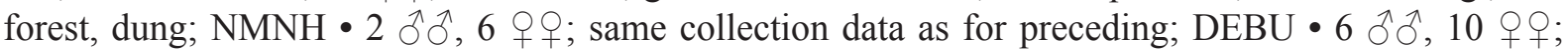
Nassau Co., Jacksonville, Cary State Forest; 10-18 Apr. 1989; Marshall and Swann leg.; dead squirrel, pan trap; DEBU • 11 ठ઼ $\widehat{\jmath}, 10$ 우; Nassau Co., nr Amelia; 3-15 Apr. 1971; A. Newton leg.; dung; NMNH

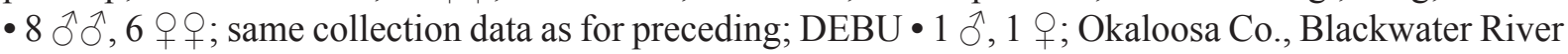
National Forest, 1 mi. N of Holt, Turkey Oak; 23 Oct. 1978; L. Stange leg.; human dung trap; DEBU • 1 J; Polk Co., Route 27, 7 mi. N of Route 14; 2-3 Nov. 1983; R.E. Woodruff leg.; pig dung trap; DEBU

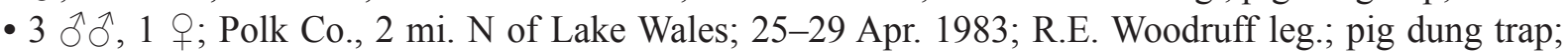

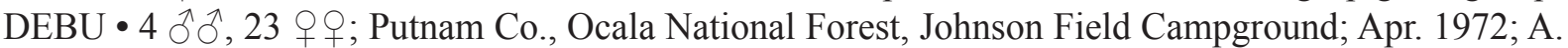
Newton leg.; mixed hardwood, dung trap; DEBU • 5 ๙ิ ô, 3 우; Sarasota Co., Myakka River State Park; 21 May 1982; R.E. Woodruff leg.; pig dung trap; DEBU • 3 $\widehat{\jmath}, 3$ 우; Putnam Co., Myakka River State Park; 22-23 May 1982; R.E. Woodruff leg.; pig dung trap; DEBU • 3 $\widehat{\jmath}, 1$ ○; Volusia Co., Tomoka State Park; 20 Jun. 1984; S.A. Marshall leg.; dung trap; DEBU • 9 $\widehat{\jmath}, 13$ 우 + ; same collection data as for preceding but mushroom trap; DEBU $\bullet 1$ \%; Hernando/Sumter Cos, Withlacoochee St. Forest, Croom

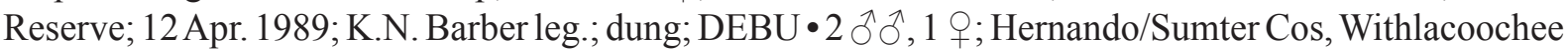




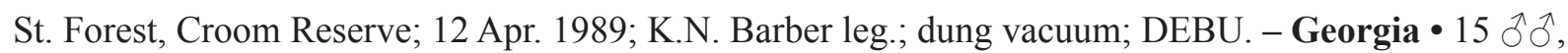
20 우; Charlton Co., 14 mi. N of Folkston; 10-18 Apr. 1989; J.E. Swann leg.; pan trap, human dung; DEBU • 1 त, 1 q; Clinch Co., US 441, 8 mi. S of Fargo; 5-25 Jun. 1984; S.A. Marshall leg.; dung trap; DEBU $\bullet 2$ ô;; Rabun Co., Chatahoochee State Forest, US 441 N of Turnerville; 5-25 Jun. 1984; S.A.

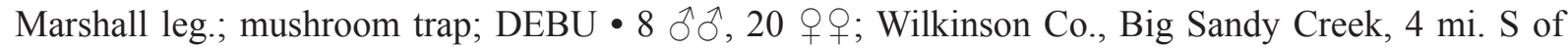

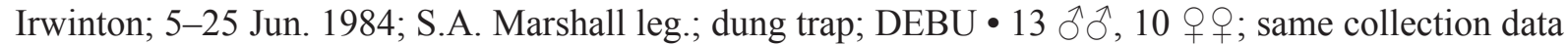
as for preceding; FIT nr dung; DEBU. - Louisiana • 1 Oे, 1 क ; East Baton Rouge Parish; 9 Apr. 1999; E.J. Watson leg.; swine carrion pitfall; DEBU -3 ô, 1 क ; Grant Parish, Stuart Lake Campground, 18 km N of Alexandria; 19 May-17 Aug. 1983; S. and J. Peck leg.; forest, FIT; DEBU • 1 o, 2 우; "Jackson Co., LA, 1.5 mi. SE Paint Rock Nat. Cave"; 9 Jul. 1967; S. Peck leg.; rat dung, Berlese \#77; DEBU. - Missouri • 1 क; Texas Co., 10.5 mi. NW of Licking, unnamed cave \#1; 14 May 1980; J.E. Gardner leg.; DEBU. - Mississippi • 5 ऽิ $\widehat{\jmath}, 8$ 우; Claiborne Co., Owen’s Creek, mi. 52, 19 km NE of

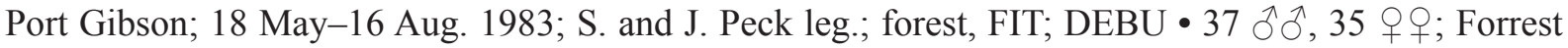
Co., Sweet Bay Bog, 6 mi. W of Wiggins; 5-8 May 1994; S.A. Marshall leg.; sphagnum, dung trap; DEBU • 1 ○; Scott Co., Forest; 5-9 May 1994; S.A. Marshall leg.; pans in prairie edge; DEBU. - North Carolina • 2 q ; Jackson Co., Cullowhee, Cane Creek; 2300 ft a.s.1.; 5-25 Jun. 1984; S.A. Marshall leg.; riparian dung trap; DEBU 1 § ; Jackson Co., Cullowhee; $5-28$ Jun. 1984; S.A. Marshall leg.; FIT; DEBU • 1 ภ, 5 क 9 ; Samson Co., Falcon at I95; 28 Sep.- 4 Oct. 1983; R. Woodruff leg.; pig dung; DEBU. - New Mexico • 1 \%; Eddy Co., 30 mi. WSW of Carlsbad, Sitting Bull Falls; $4600 \mathrm{ft}$ a.s.l.; 23-27 Jul. 1975; S. Peck leg.; dung; DEBU. - Oklahoma • 1 đ ; Lattimer Co., 5 mi. W of Red Oak; 5-11 Jun. 1977; K. Stephan leg.; dung trap; DEBU. - South Carolina • 1 ô, 1 q; Barnwell Co., Barnwell

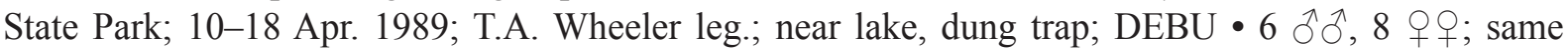
collection data as for preceding; S.A. Marshall leg.; mushroom traps in oak forest; DEBU 1 औ, 3 q $ᄋ$; Colleton Co., Colleton State Park; 27 Sep.- 5 Oct. 1983; R.E. Woodruff leg.; pig dung trap; DEBU • 1 §ิ, 4 우; Georgetown Co., Hobcaw Barony, Belle Baruch Marine Field Lab, Crabhaul Road near

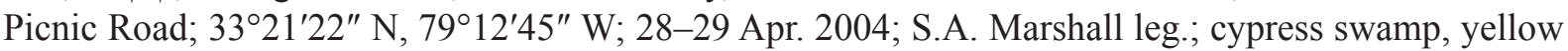
pans; DEBU • 11 ठิ $\widehat{\jmath}, 18$ 우; Georgetown Co., Hobcaw Barony, Belle Baruch Marine Field Lab, Crabhaul Road near Picnic Road; 33²1'22" N, 79¹2'45" W; 29-30 Apr. 2004; Cheung and Macleod

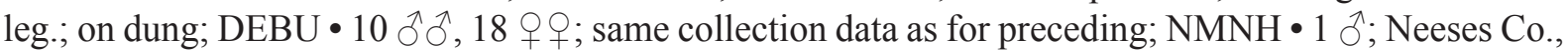
13 mi. W of Orangeburg; 27 Mar.1980; S.A. Marshall leg.; DEBU. - Tennessee 1 ơ; Henderson Co., Natchez Trace State Park; 1000 ft a.s.1.; 18 Jun.-13 Jul. 1972; A. Newton leg.; dung; FMNH. - Texas • 3 우우; Angelina Co., Angelina National Forest, Boykin Cemetery; 13-17 Jun. 1993; S.A. Marshall leg.; spring fen, dung; DEBU $\bullet 1$ O , 6 우; Angelina Co., Angelina National Forest, Bouton Lake Campground; 12-14 Jun. 1993; S.A. Marshall leg.; dung/vac traps; DEBU • 1 đో; Brazos Co., College Station, Lick

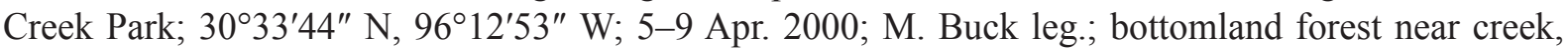
Malaise trap; DEBU $\bullet 1$; same collection data as for preceding; post oak savanna by creek, Malaise trap; DEBU • 1 q; Montgomery Co., 4.5 mi. N of Montgomery; 2 May-17 Jun. 1987; R.S. Anderson;

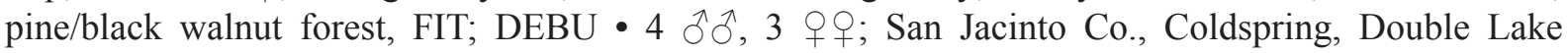
Campground; 22 May-16 Aug. 1983; S. and J. Peck leg.; FIT; DEBU • 3 우우 S San Jacinto Co., Double Lake Campground, 5 km S of Coldspring; 22-24 May 1983; S. Peck leg.; forest, carrion trap; DEBU •

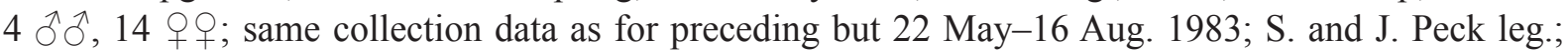
forest, FIT; TAMU 11 우; Tyler Co., Kirby State Forest, dung traps; 12-17 Jun. 1993; S.A. Marshall leg.; DEBU • 4 o $\circ$; Tyler Co., Bouton Lake; 13-17 Jun. 1993; S.A. Marshall leg.; dung/vac traps; DEBU.

VENEZUELA - Aragua • 1 क ; Henri Pittier National Park, Rancho Grande, La Toma; 1150 m a.s.l.; 9 Apr. 1994; L. Masner leg.; DEBU • 3 q o ; Maracay, Rancho Grande; 1200 m a.s.1.; 1-10 Aug. 1987;

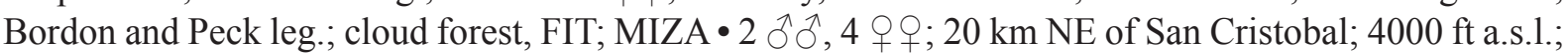
18-22 May 1974; S. Peck leg.; dung trap; MIZA. - Bolívar • 3 ふ઼ đ; km 40 on Santa Elena Icabaru Road; 1000 m a.s.1.; 4-6 Aug. 1986; B.D. Gill leg.; DEBU • 1 đ̊; same collection data as for preceding; CNCI 


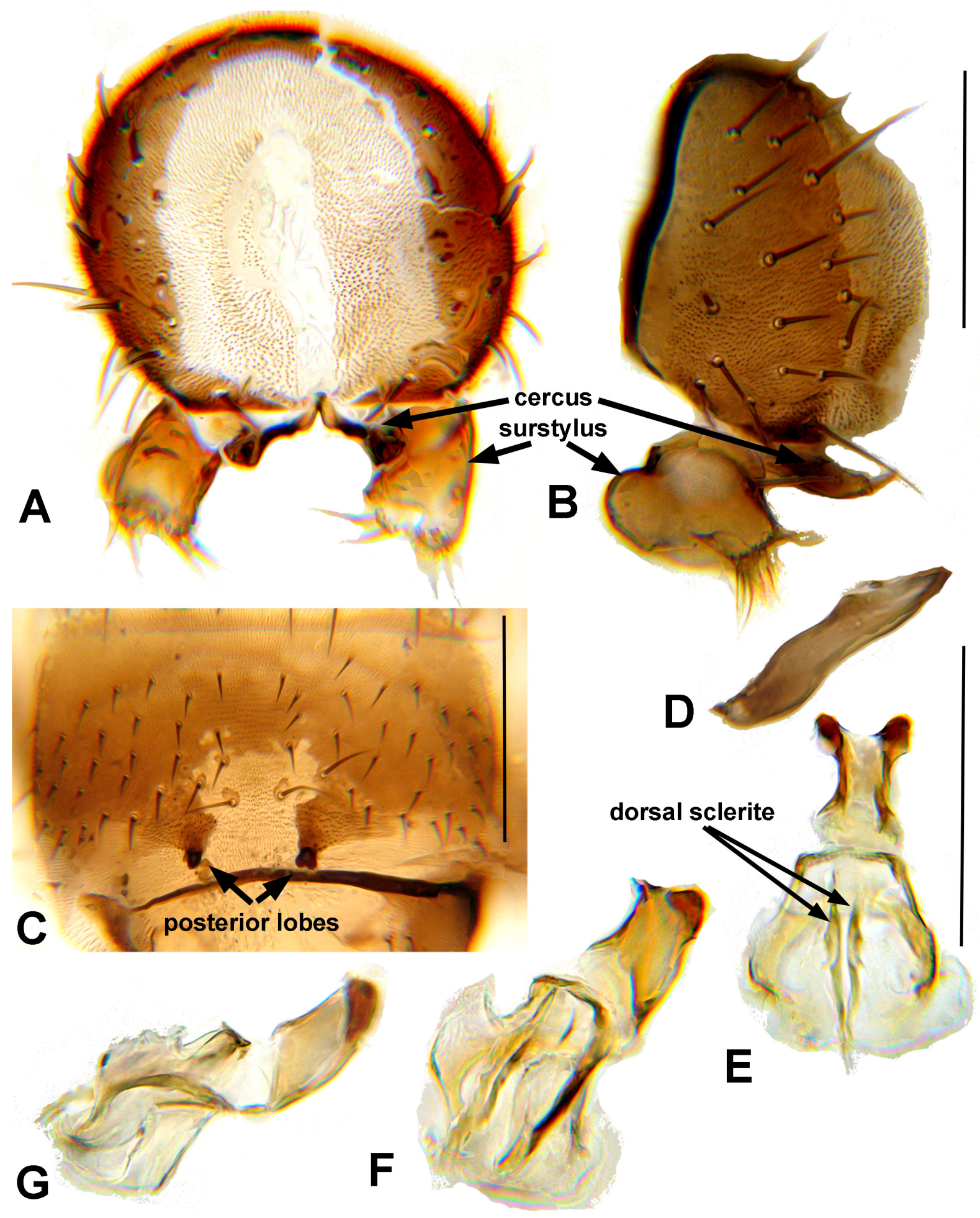

Fig. 9. Rudolfina exuberata sp. nov., male terminalia (debu00242299). A. Epandrium, surstylus and cercus, caudal view. B. Same as preceding, lateral view. C. Sternite 5, ventral view. D. Postgonite, close up lateral view. E. Phallus, dorsal view. F. Same as preceding, dorsolateral view. G. Same as preceding, lateral view. Scale bars: A-B $=0.10 \mathrm{~mm} ; \mathrm{C}=0.10 \mathrm{~mm}$; $-\mathrm{F}=0.10 \mathrm{~mm}$. 


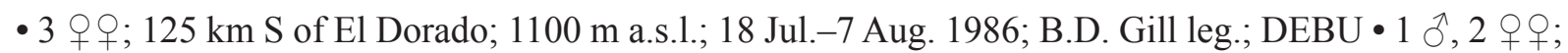
135 km S of El Dorado; 1400 m a.s.1.; 20 Jul.-7 Aug. 1986; B. Gill leg.; dung traps; CNCI • 1 ô, 1 O; 10 km S of El Dorado; 200 m a.s.l.; 17 Jul.-7 Aug. 1986; B.D. Gill leg.; DEBU • 1 §’; 33 km S of El Dorado; 220 m a.s.1.; 2-7 Aug. 1986; B.D. Gill leg.; CNCI • 1 +, km 40 on Santa Elena Icabaru Road; 220 m a.s.l.; 4-6 Aug. 1986; B.D. Gill leg.; MIZA • 1 क; 22 km S of El Dorado; 25 Jun.-12 Jul. 1987; S. and J. Peck leg.; lowland rainforest, FIT; DEBU.

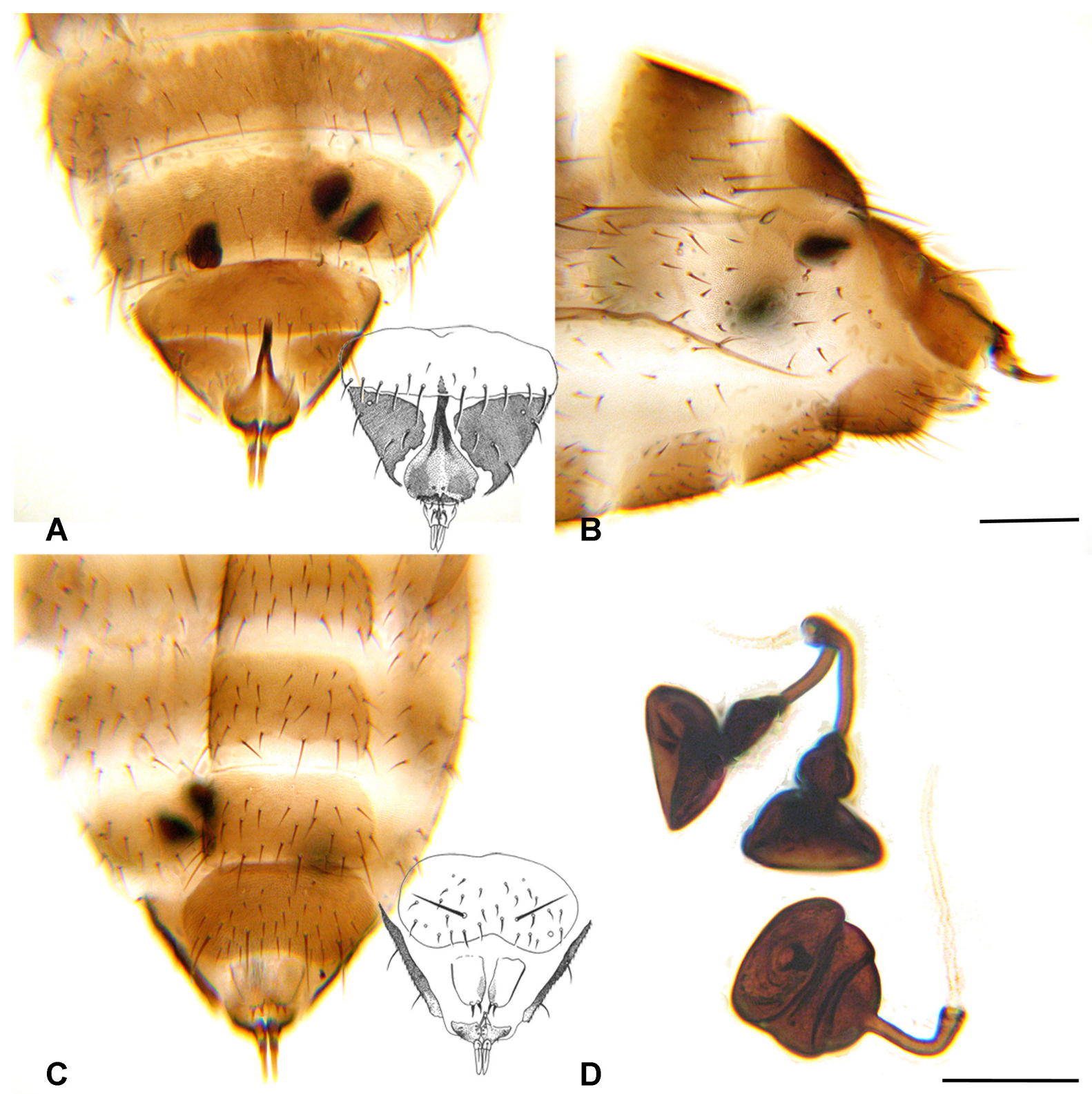

Fig. 10. Rudolfina exuberata sp. nov., female terminalia (A-C: debu00242299; D: debu00242286). A. Terminal abdominal segments, dorsal view. B. Same as preceding, lateral view. C. Same as preceding, ventral view. D. Spermathecae. Scale bars: $A-C=0.10 \mathrm{~mm}$; $=0.03 \mathrm{~mm}$. 


\section{Description}

Body. Length 1.5-2.0 mm. Eye height $2.0 \times$ genal height. Head with 4 interfrontal setae and row of 5-6 small setulae on inner margin of orbital plate. Gena with 1 strong seta and 5-7 smaller setulae. Acrostichal setulae in 6-8 rows. Costagial seta extending to humeral break. Second costal sector $0.3-$ $0.4 \times$ third costal sector. Length of $\mathrm{M}_{1}$ between crossveins $\mathrm{dm}-\mathrm{cu}$ and $\mathrm{r}-\mathrm{m} 1.2 \times \mathrm{dm}-\mathrm{cu} ; \mathrm{CuA}_{1}$ stub vein $\sim 3.0 \times \mathrm{M}_{1}$ stub vein. Male mid femur with single ventrobasal seta. Male mid tibia with as1 enlarged preapical seta and 1 smaller seta near midlength. Female mid tibia with midventral seta.

Male ABdomen (Fig. 9). Sternite 5 with deep irregular posteromedial emargination flanked by blunt lobe on each side; emargination extending anteriorly $1 / 3$ length of sternite. Transverse part of sternite 6 straight. Surstylus (in lateral view) bilobed; anterior lobe rounded and bare; posterior lobe rounded but apically flattened and with 5-10 setae. Cercus projecting posteriorly, with apical seta. Postgonite simple, slightly sinuate, with small robust seta apically and 1-2 setulae along length. Dorsal sclerite of distiphallus (Fig. 9F-H) without distinct swellings.

Female ABdomen (Fig. 10). Tergite 7 with posterior margin entire. Medial part of tergite 8 elongate, narrower anteriorly, posteriorly closely approximated with epiproct. Epiproct tulip-shaped, heavily sclerotized; longitudinally lightly sclerotized on apical half. Cercus strap-like, length $\sim 0.5 \times$ epiproct. Sternite 7 wider than sternite 6; posterior margin with broad, shallow emargination. Spermathecae bilobed, constricted near midlength; distal portion cup-shaped, basal portion ovoid and narrowed before duct junction; spermatheca stem as long as spermathecal width.

\section{Remarks}

Rudolfina exuberata sp. nov. is the only species of Rudolfina that regularly occurs at low elevations and is widespread throughout South America, Central America and the southern United States.

Rudolfina exuberata sp. nov. resembles R. pauca sp. nov. in both sexes, but these species are distinguished by characters of the surstylus, male cercus and spermathecae.

Rudolfina howdeni sp. nov.

urn:lsid:zoobank.org:act:0B9431DE-EFE1-41EC-B9B3-39303EDE435E

Figs 1A, 11-12, 21B

\section{Etymology}

This species name, which has been a manuscript name since Marshall (1982), is a patronym for Dr Henry Howden. Henry was a great entomologist and friend who advised SM during his M.Sc. at Carleton University; we regret that he did not live to see this beautiful species published.

\section{Material examined}

Holotype

MEXICO • ^`; Oaxaca, 6.6 mi. N of Ixtlan de Juarez; 8300 ft a.s.1.; 10-18 Aug. 1973; A. Newton leg.; oak woodland, human dung; FMNH debu01086096.

\section{Paratypes}

MEXICO - Hidalgo • 1 万’; 7 mi. SW of Tenango de Doria; $7000 \mathrm{ft}$ a.s.1.; 2-6 Jul. 1971; A. Newton leg.; cloud oak forest, human dung; DEBU. - Jalisco • $6 \hat{\jmath} \widehat{\jmath}, 1$ क; $14 \mathrm{mi}$. W of Atenquique; $7900 \mathrm{ft}$ a.s.l.; A. Newton leg.; hardwood forest, dung; CASC $\bullet 6 \hat{\delta}$; same collection data as for preceding; FMNH

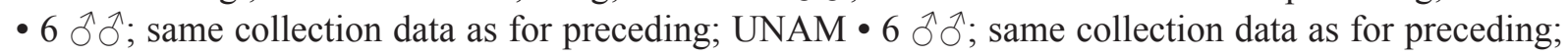

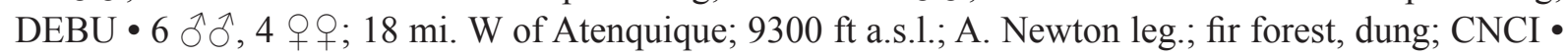




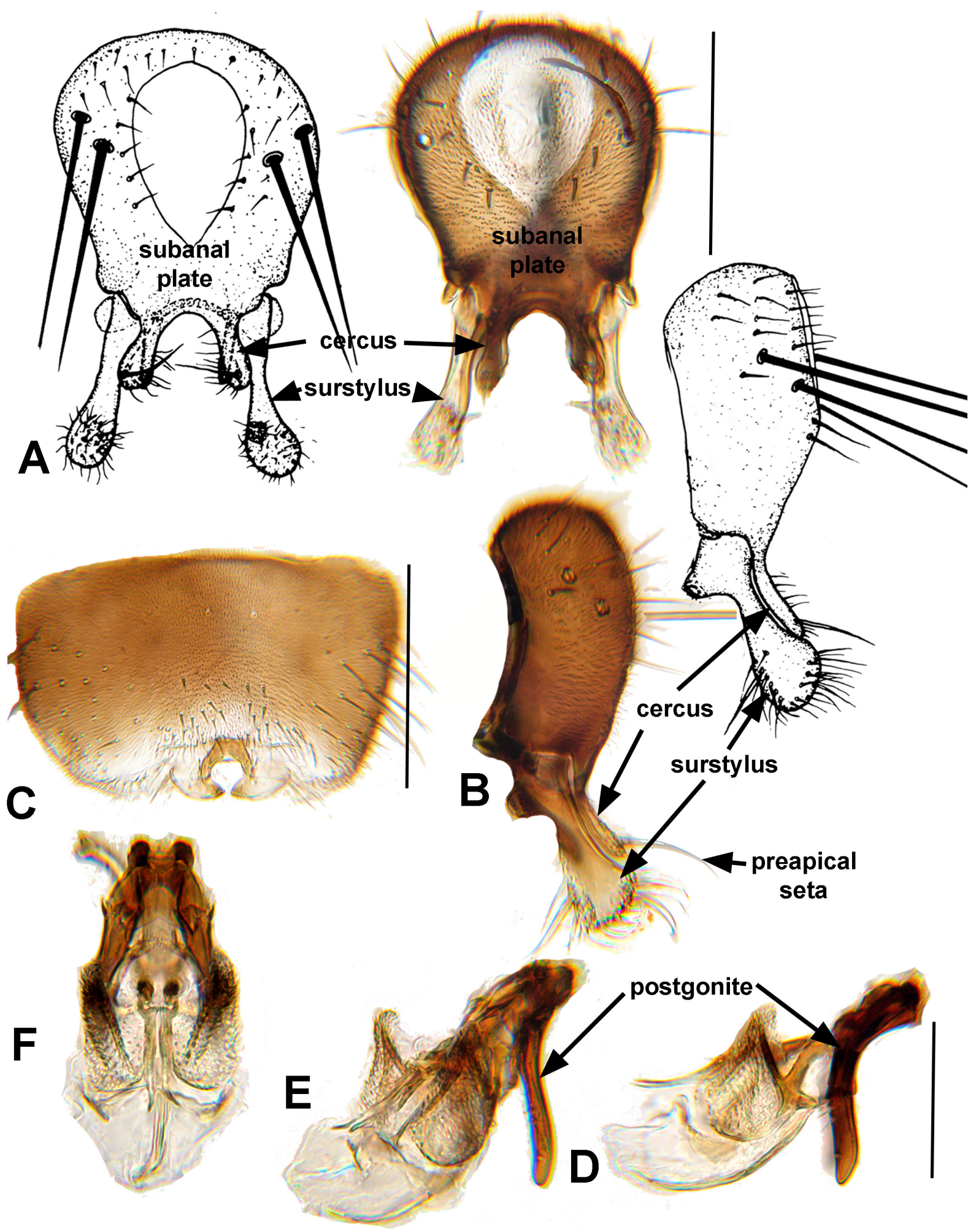

Fig. 11. Rudolfina howdeni sp. nov., male terminalia (debu01086163). A. Epandrium, surstylus and cercus, caudal view. B. Same as preceding, lateral view. C. Sternite 5, ventral view. D. Phallus and postgonites, lateral view. E. Same as preceding, dorsolateral view. F. Same as preceding, dorsal view. Scale bars: $A-B=0.10 \mathrm{~mm} ; \mathrm{C}=0.10 \mathrm{~mm}$; $-\mathrm{F}=0.05 \mathrm{~mm}$. 


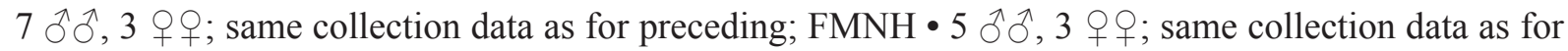

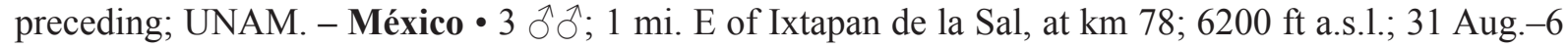
Sep. 1971; A. Newton leg.; Trop/Dec/Jun., human dung; DEBU • 5 đ̂े ô, 1 o; 5 mi. E of Santa Marta, at km 8.5; $10100 \mathrm{ft} \mathrm{a.s.1.;} 29$ Aug.-4 Sep. 1971; A. Newton leg.; fir forest, human dung; DEBU. - Morelos - 2 우; 4 mi. W of Tres Cumbres, at km 6; 8900 ft a.s.l.; 29 Aug.-4 Sep. 1971; A. Newton leg.; oak,

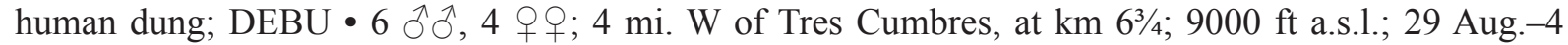

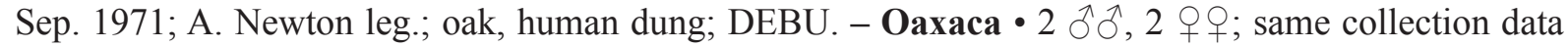

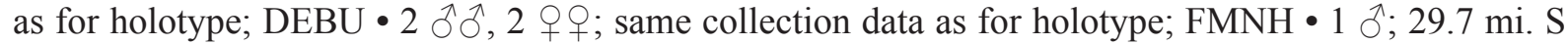
of Valle Nacional; $6800 \mathrm{ft}$ a.s.1.; 11-17 Aug. 1973; A. Newton leg.; cloud forest, dung; DEBU • 1 ऽ; 2.0 mi. W of Jct. Mex. 175-Yuvila Rd; 9500 ft a.s.1.; 8-19 Aug. 1973; A. Newton leg.; oak-pine, dung;

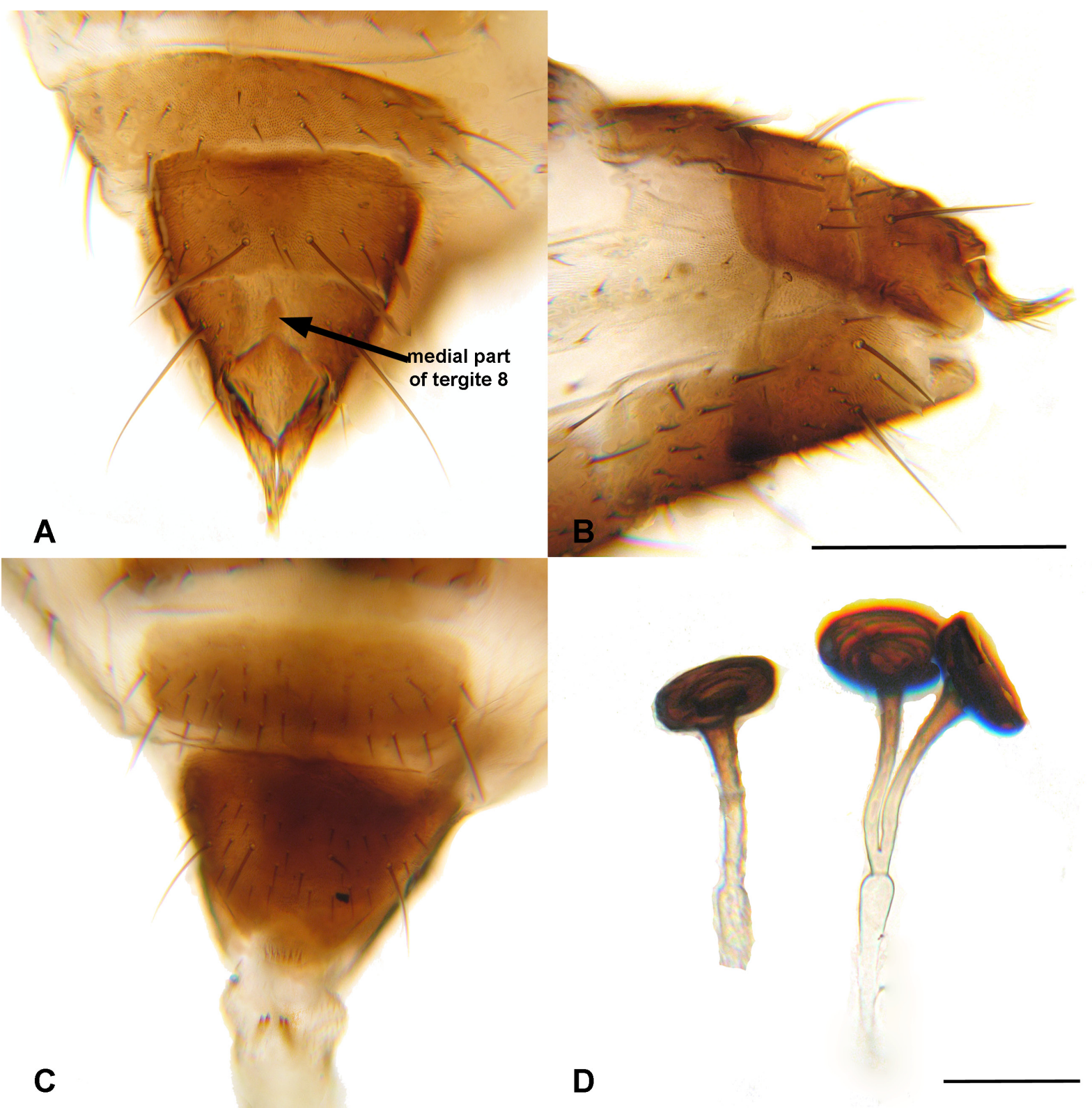

Fig. 12. Rudolfina howdeni sp. nov., female terminalia (debu1086100). A. Terminal abdominal segments, dorsal view. B. Same as preceding, lateral view. C. Same as preceding, ventral view. D. Spermathecae. Scale bars: $\mathrm{A}-\mathrm{C}=0.30 \mathrm{~mm}$; $\mathrm{D}=0.04 \mathrm{~mm}$. 
DEBU • 1 đ̧; 4.1 mi. W of Jct. Mex. 175-Yuvila Rd; 9300 ft a.s.1.; 8-19 Aug. 1973; A. Newton leg.; oak-fir-pine forest; DEBU 11 万; 5 mi. E of Jct. Mex. 175-Yuvila Rd; $7600 \mathrm{ft}$ a.s.1.; 9-19 Aug. 1973; A. Newton leg.; oak, pine, dung; DEBU 1 ơ; 20 mi. N of Ixtlan; 2835 m a.s.1.; 24-27 Jul. 1971; A. Newton leg.; oak-pine; DEBU. - Veracruz • 1 \%; Ciudad Mendoza, 8.2 mi. W on Mex. 150D; $6200 \mathrm{ft}$ a.s.1.; 27 Jul.-3 Aug. 1973; A. Newton leg.; oak woodland, carrion; DEBU.

\section{Description}

BoDy. Length: 1.6-2.0 mm. Eye height $2.0 \times$ genal height. Head with 4-5 interfrontal setae and row of 6-8 small setulae on inner margin of orbital plate. Gena with 2 setae and 6-8 setulae. Acrostichal setulae in 4-6 rows. Costa with costagial seta extending to subcostal break. Second costal sector $\sim 0.85 \times$ third costal sector. Length of $\mathrm{M}_{1}$ between crossveins $\mathrm{dm}-\mathrm{cu}$ and $\mathrm{r}-\mathrm{m} \sim 2.0 \times \mathrm{dm}-\mathrm{cu}$; $\mathrm{CuA}_{1}$ stub vein $\sim 7.0 \times \mathrm{M}_{1}$ stub vein. Male mid femur with 10-15 setae in ovoid ventrobasal cluster. Male mid tibia with ventral comb of $6-7$ robust, short setae on apical $1 / 2$.

MALE ABDomen (Fig. 11). Posterior margin of sternite 5 with pair of large medially-projecting blunt teeth on each side of emargination; emargination extending $\sim 1 / 4$ length and $\sim 1 / 8$ width of sternite 5 ; emargination lined with sclerotized strip. Transverse part of sternite 6 weakly arcuate. Epandrium with pair of long lateral setae, 3-4 smaller pairs of setae along lower margin of anal opening and several other small setae scattered over the surface; subanal plate narrow but complete. Surstylus (in lateral view) with small hirsute anterior lobe and elongate clavate posterior lobe $(\sim 4.0 \times$ length of anterior lobe); posterior lobe with 4-5 large setae and numerous smaller setae apically, and 2-3 bifurcating setae on inner surface. Cercus elongate, clavate, projecting ventrally. Postgonite elongate, narrowly rounded apically. Dorsal sclerite of distiphallus (Fig. 11F-H) not swollen along length and not extending beyond apex of acrophallus.

Female abdomen (Fig. 12). Posterior margin of tergite 7 entire or slightly emarginate medially. Medial part of tergite 8 elongate (length $\sim 1.5-3.0 \times$ width), with anterior margin rounded (some specimens with posterior half poorly sclerotized and difficult to see). Epiproct diamond-shaped. Cercus as long as epiproct, with elongate preapical seta. Sternite 7 entire. Spermathecae cup-shaped and short, with sclerotized duct $\sim 3.5 \times$ length of spermatheca.

\section{Remarks}

The male sternite 5, epandrium, surstylus and cercus are unique within Rudolfina.

Rudolfina megepandria sp. nov. urn:lsid:zoobank.org:act:16E7249F-BC7E-4CD0-AFCB-60A5C98CFB43

Figs 4, 13, 21B

\section{Etymology}

The specific name, which has been a manuscript name since Marshall (1982), refers to the unusually large epandrium.

\section{Material examined}

Holotype

MEXICO • ${ }^{\top}$; Jalisco, 18 mi. W of Atenquique; 9300 ft a.s.l.; A. Newton leg.; fir forest, dung; FMNH debu01086084. 


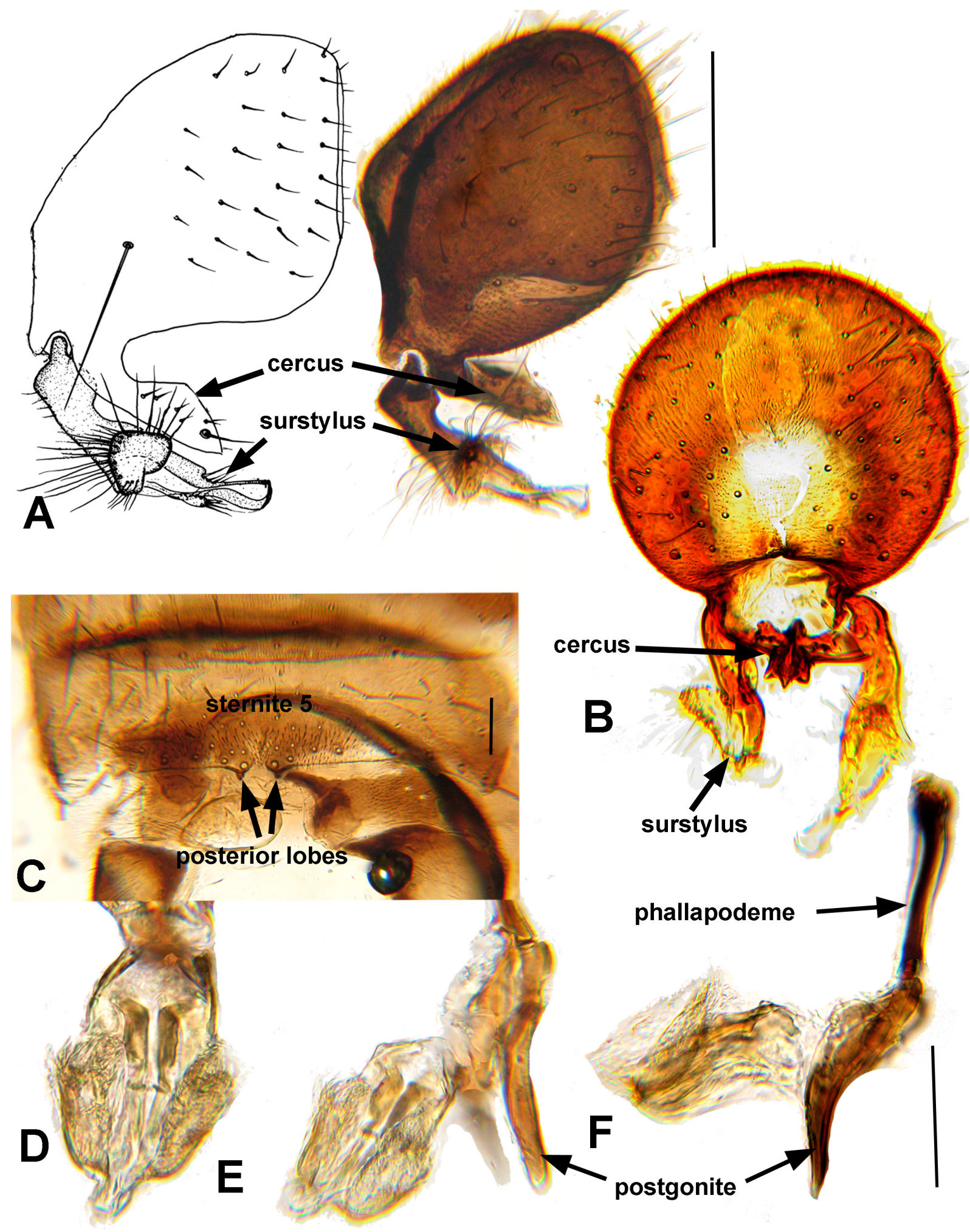

Fig. 13. Rudolfina megepandria sp. nov., male terminalia (debu01086085). A. Epandrium, surstylus and cercus, lateral view. B. Same as preceding, caudal view. C. Sternites $4-7$, ventral view. D. Phallus and postgonite, dorsal view. E. Phallus, postgonite and phallapodeme, dorsolateral view. F. Same as preceding, lateral view. Scale bars: $A-B=0.20 \mathrm{~mm} ; C=0.05 \mathrm{~mm} ; \mathrm{D}-\mathrm{F}=0.05 \mathrm{~mm}$. 
Paratypes

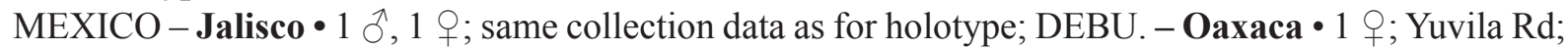
9400 ft a.s.l.; 9-19 Aug. 1973; A. Newton leg.; mesic oak, carrion; FMNH.

\section{Description}

BoDy. Length: 1.9-2.2 mm. Eye height $2.5 \times$ genal height. Head with 3 interfrontal setae and row of 4-5 small setulae on inner margin of orbital plate. Gena with 2 strong setae and 7-8 smaller setulae. Acrostichal setulae in 4-6 rows. Costagial seta extending to subcostal break. Second costal sector $0.75-$ $0.8 \times$ third costal sector. Length of $\mathrm{M}_{1}$ between crossveins dm-cu and $\mathrm{r}-\mathrm{m}-3.0 \times \mathrm{dm}-\mathrm{cu} ; \mathrm{CuA}_{1}$ stub vein $\sim 10.0 \times \mathrm{M}_{1}$ stub vein $\left(\mathrm{M}_{1}\right.$ stub vein extremely reduced). Male mid femur with 14-15 strong setae in ventrobasal cluster. Male mid tibia with ventral comb of $4-5$ robust, short setae on apical $1 / 2$. Mid tibia without midventral seta (both sexes).

MALE ABDOMEN (Fig. 13). Sternite 5 posterior margin with 2 small nipple-like teeth on each side of small emargination; emargination $\sim 1 / 15$ width and $\sim 1 / 9$ length of sternite 5 . Transverse part of sternite 6 arcuate. Epandrium swollen, broader than preceding abdominal segments; subanal plate broad, complete. Surstylus with posterior lobe elongate, length $\sim 4.0 \times$ basal width, with a hirsute medial lateral lobe; apex truncate. Cercus elongate-conical, projecting posteriorly. Postgonite elongate, narrowly rounded distally and narrow basally. Dorsal sclerite of distiphallus (Fig. 13D-F) without dorsal swellings and not extending beyond apex of acrophallus.

Female ABdomen (Fig. 4). Tergite 7 with posterior margin entire. Medial part of tergite 8 elongate, length $\sim 2.0-3.0 \times$ width, with posterior margin emarginate. Epiproct broadly rounded anteriorly. Cercus $\sim 0.75 \times$ length of epiproct; fused anterolaterally to epiproct. Sternite 7 with posterior margin broadly rounded. Spermathecae cup-shaped; paired spermathecae with stems $\sim 1.5 \times$ width of spermatheca.

\section{Remarks}

Rudolfina megepandria sp. nov. is diagnosed by its swollen male epandrium and distinctively shaped sternite 5 . It is most closely related to $R$. bucki sp. nov., which has a less pronounced epandrium and differs in details of the surstylus and cercus.

Rudolfina newtoni sp. nov. urn:1sid:zoobank.org:act:02FE2BAE-7EEF-4889-A3DD-9540B9DE96C1

Figs $14-15,21 \mathrm{C}$

\section{Etymology}

The species name is a patronym for Dr Alfred Newton, the collector of all known specimens of this species.

\section{Material examined}

\section{Holotype}

MEXICO • ${ }^{\text {; }}$; Oaxaca, 4.1 mi. W of Jct. Mex. 175-Yuvila Rd; 9300 ft a.s.1.; 8-19 Aug. 1973; A. Newton leg.; oak-fir-pine forest; FMNH debu01086218.

\section{Paratypes}

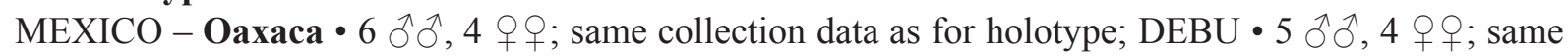
collection data as for holotype; FMNH $11 \partial^{\lambda}, 1$ क; $25 \mathrm{mi}$. N of Ixtlan; $17^{\circ} 33^{\prime} 0^{\prime \prime} \mathrm{N}, 96^{\circ} 31^{\prime} 12^{\prime \prime} \mathrm{W} ; 9100 \mathrm{ft}$ a.s.1.; 23-29 Jul. 1971; A. Newton leg.; oak-pine, human dung; FMNH • $3 \widehat{\jmath} \widehat{\jmath}, 1$ ㅇ; 20 mi. N of Ixtlan; 2835 m a.s.1.; 24-27 Jul. 1971; A. Newton leg.; oak-pine; DEBU • 1 क; 28 mi. N of Ixtlan; $9500 \mathrm{ft}$ 


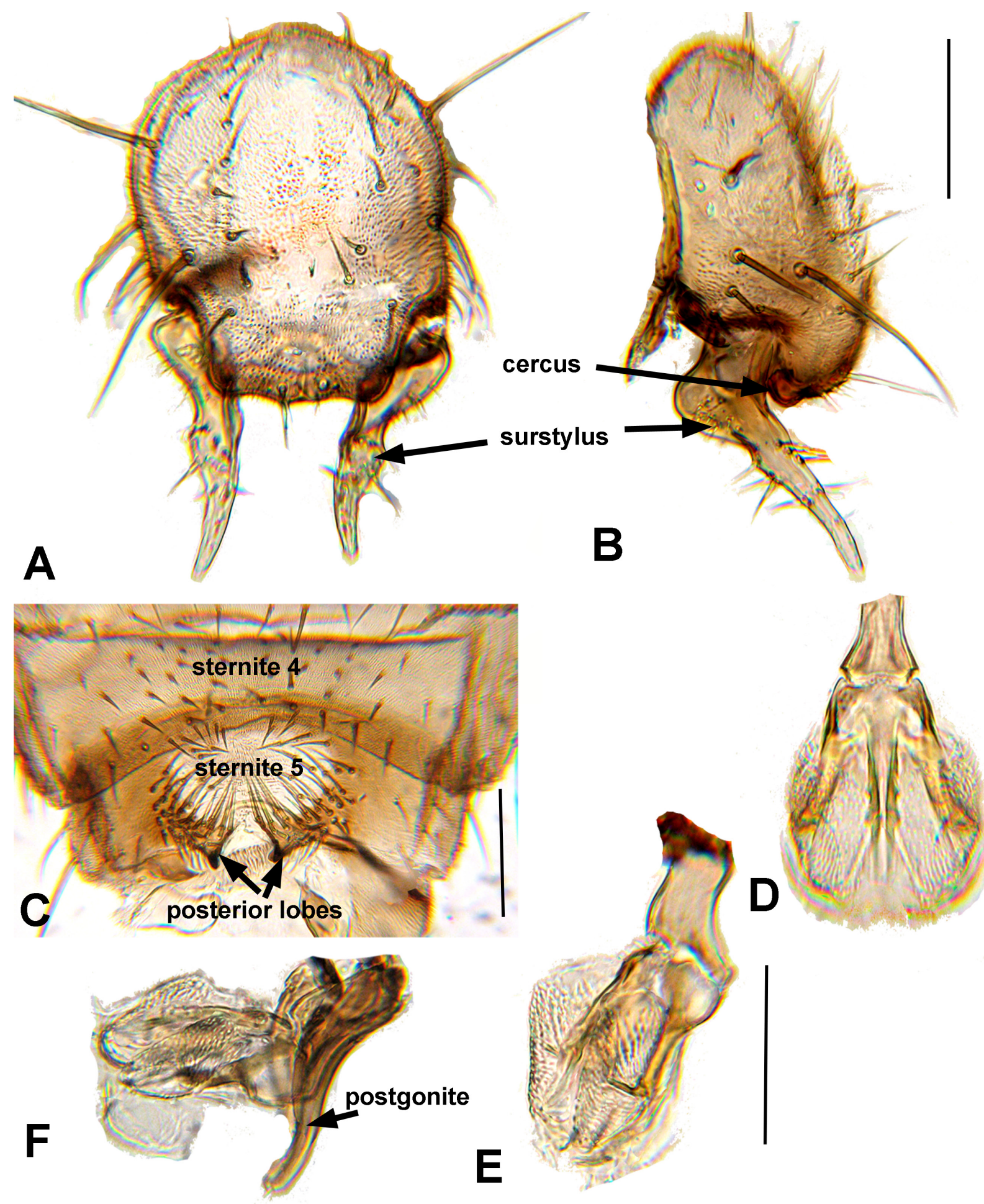

Fig. 14. Rudolfina newtoni sp. nov., male terminalia (A-E: debu01086234; F: debu01086234). A. Epandrium, surstylus and cercus, caudal view. B. Same as preceding, lateral view. C. Sternites 4 and 5, ventral view. D. Phallus, dorsal view. E. Phallus, dorsolateral view. F. Phallus and postgonite, lateral view. Scale bars: $A-B=0.10 \mathrm{~mm} ; C=0.10 \mathrm{~mm}$; $-\mathrm{F}=0.05 \mathrm{~mm}$. 


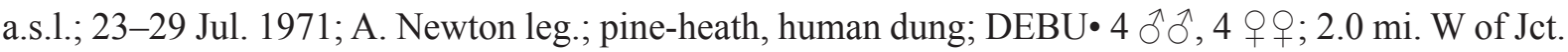
Mex. 175-Yuvila Rd; 9500 ft a.s.l.; 8-19 Aug. 1973; A. Newton leg.; oak-pine, dung; DEBU • 4 $\widehat{\partial}$, 5 우영 same collection data as for preceding; UNAM • 2 우; 1.7 mi. W of Jct. Mex. 175-Yuvila Rd;

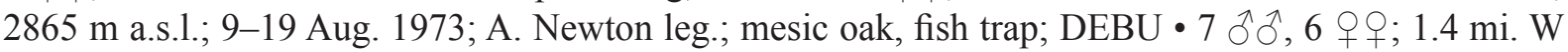
of Jct. Mex. 175-Yuvila Rd; 9300 ft a.s.1.; 9-19 Aug. 1973; A. Newton leg.; mesic oak forest; DEBU • $3 \widehat{\partial} \hat{\jmath}, 2$ 우; same collection data as for preceding; dung; DEBU.

\section{Description}

BoDy. Length: 1.6-2.0 mm. Eye height $1.5 \times$ genal height. Head with 4 interfrontal setae and row of 6-7 small setulae on inner margin of orbital plate. Gena with 2 strong setae and 6-8 smaller setulae.

A
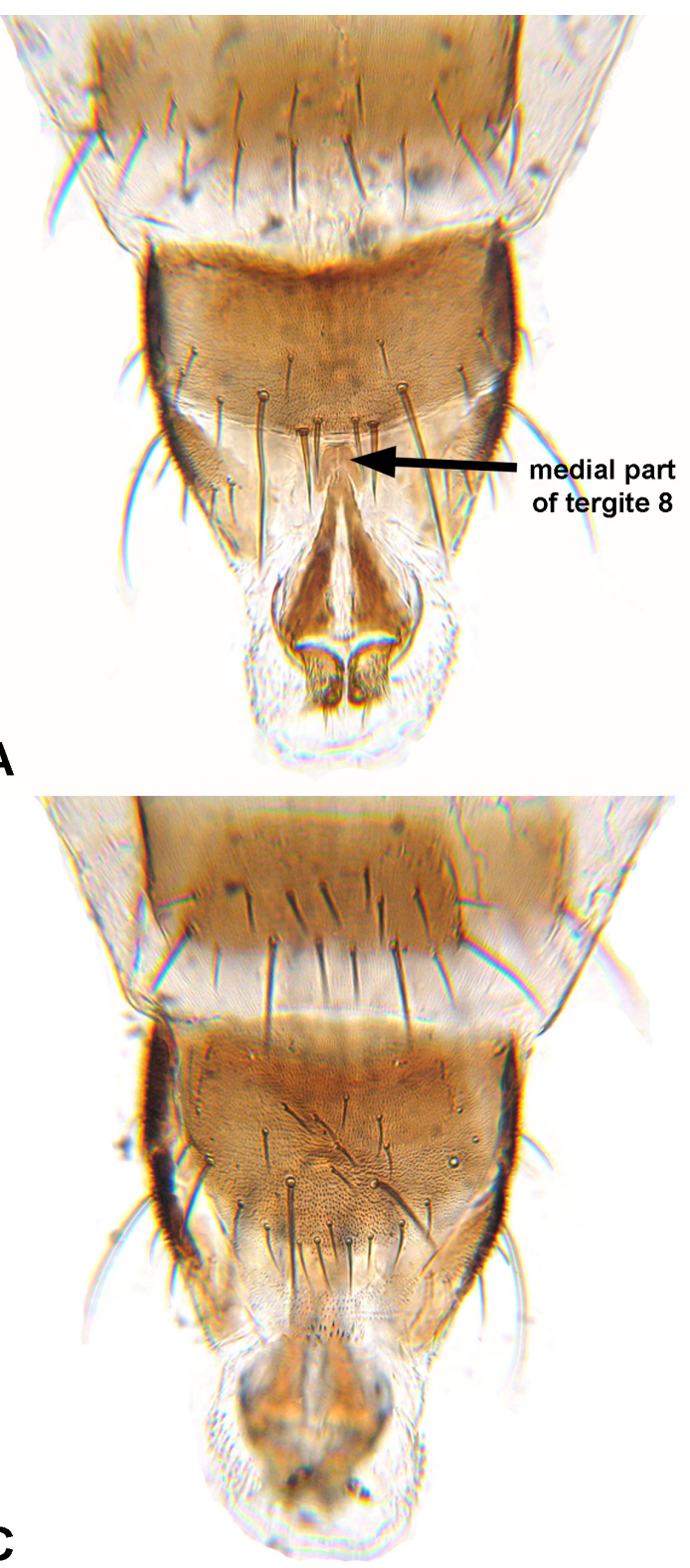

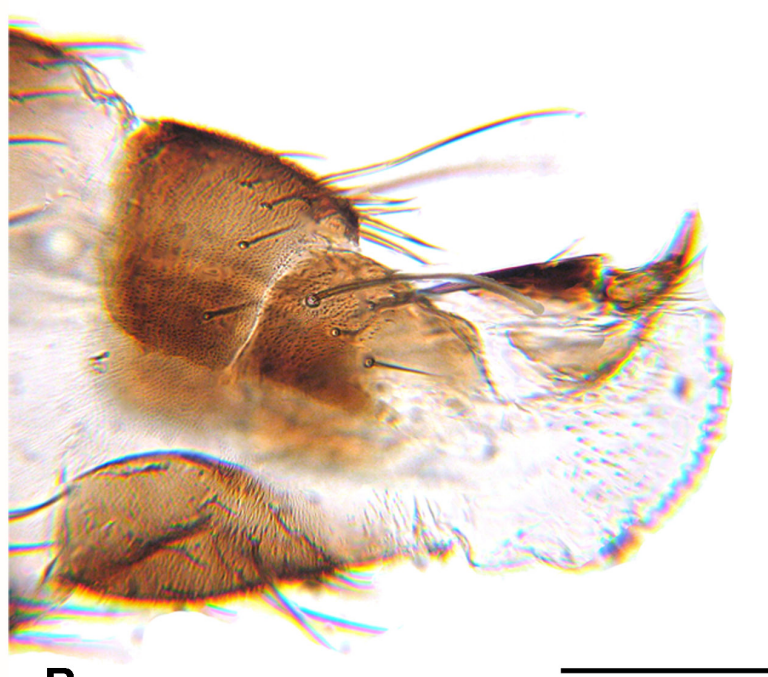

B

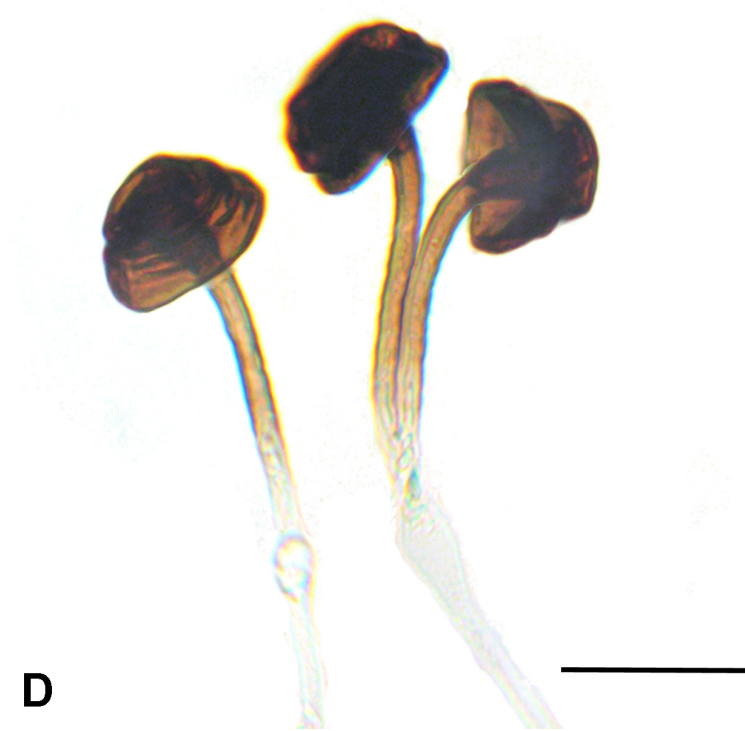

Fig. 15. Rudolfina newtoni sp. nov., female terminalia (debu01086226). A. Terminal abdominal segments, dorsal view. B. Same as preceding, lateral view. C. Same as preceding, ventral view. D. Spermathecae. Scale bars: $\mathrm{A}-\mathrm{C}=0.10 \mathrm{~mm} ; \mathrm{D}=0.04 \mathrm{~mm}$. 
Acrostichal setulae in 6-8 rows. Costagial seta extending slightly beyond humeral break. Second costal sector $\sim 1.2 \times$ as long as third. Length of $\mathrm{M}_{1}$ between crossveins dm-cu and r-m $2.0 \times \mathrm{dm}-\mathrm{cu} ; \mathrm{CuA}_{1}$ stub vein $\sim 4.0 \times \mathrm{M}_{1}$ stub vein. Male mid femur with 20-21 strong setae in ventrobasal cluster. Male mid tibia with ventral comb of 8 robust setae on apical $1 / 2$.

Male abdomen (Fig. 14). Sternite 5 posterior margin with two distinct teeth on each side of large emargination; emargination forming a broad circular desclerotized area, $\sim 1 / 3$ width of sclerite and extending anteriorly $\sim 3 / 4$ of length; margin of emargination with numerous setae on posterior $2 / 3$. Transverse part of sternite 6 arcuate. Epandrium with pair of long mediolateral setae, with 2-3 smaller pairs lateral to large pair; subanal plate narrowly continuous below anal opening. Surstylus (in lateral view) with anterior lobe reduced to small basal swelling; posterior lobe elongate, length $\sim 4.0 \times$ basal width, tapering to narrowly rounded apex; bent posteriorly near basal $1 \frac{1}{4}$, with $4-5$ setae on both ventral and dorsal surfaces. Cerci quadrate, weakly differentiated from each other and from epandrium, with pair of ventromedial setae. Postgonite narrow, slightly sinuate, with 4 small dorsal setulae on apical $1 / 2$. Dorsal sclerite of distiphallus (Fig. 14D-F) without swellings and not extending beyond apex of acrophallus.

Female abdomen (Fig. 15). Posterior margin of tergite 7 entire. Medial part of tergite 8 short and kidneyshaped, with posterior margin emarginate. Epiproct triangular, elongate, with mid-line desclerotized. Cercus half as long as epiproct, with flattened apical seta; narrowly fused anterolaterally to epiproct. Posterior margin of sternite 7 entire or slightly arcuate. Spermathecae inverted cup-shaped (with duct, appearing mushroom-shaped) and wrinkled; sclerotized portion of ducts $\sim 2.0 \times$ length of spermatheca; common duct of paired spermathecae poorly sclerotized.

\section{Remarks}

This species is distinguished from other Rudolfina by characters of the surstylus and male sternite 5 . The female epiproct is intermediate in shape between the strongly modified epiproct found in the $R$. exuberata group and the unmodified epiproct of other Rudolfina.

\section{Rudolfina pauca sp. nov. urn:lsid:zoobank.org:act:F085E96F-6CCB-49B0-83B8-503F18F1B472}

Figs $16-17,21 \mathrm{C}$

\section{Etymology}

The species name is the Latin for 'few'. Compared to the closely related $R$. exuberata sp. nov., which occurs throughout the Neotropical region and has been collected at over a hundred different sites, $R$. pauca sp. nov. has been infrequently collected and remains known from only five sites in Mexico and Guatemala.

\section{Material examined}

\section{Holotype}

MEXICO • đ’; México, 1 mi. NE of Tenancingo; 7100 ft a.s.l.; 31 Aug.-6 Sep. 1971; A. Newton leg.; oak-pine-madrono, human dung; FMNH debu01086244.

\section{Paratypes}

GUATEMALA - San Marcos • 1 q; San Antonio Sacatepéquez; $14^{\circ} 58^{\prime}$ N, $91^{\circ} 44^{\prime}$ W; $8000 \mathrm{ft}$ a.s.1.; 29 Sep. 1986; M.J. Sharkey leg.; DEBU.

MEXICO - Hidalgo • 1 §, 1 क; 4 mi. W of Tulancingo; $7600 \mathrm{ft}$ a.s.l.; 1-6 Jul. 1971; A. Newton leg.;

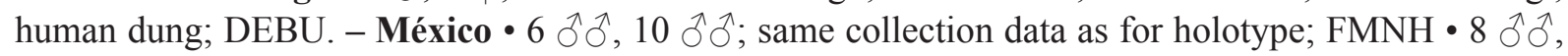




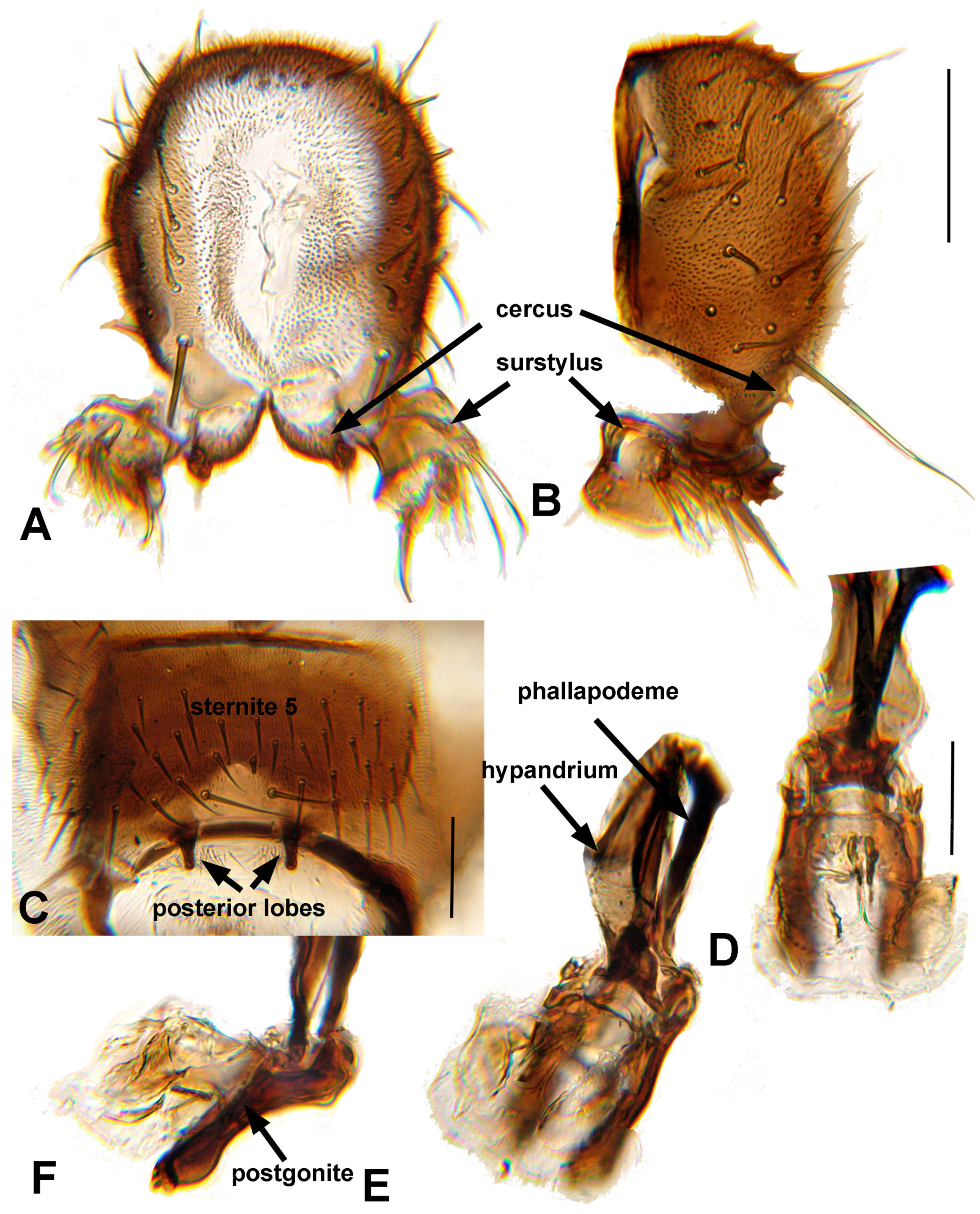

Fig. 16. Rudolfina pauca sp. nov., male terminalia (debu1086247). A. Epandrium, surstylus and cercus, caudal view. B. Same as preceding, lateral view. C. Sternite 5, ventral view. D. Phallus, postgonites and phallapodeme, dorsal view. E. Phallus, postgonites, hypandrium and phallapodeme, dorsolateral view. F. Same as preceding, lateral view. Scale bars: $A-B=0.10 \mathrm{~mm} ; C=0.10 \mathrm{~mm}$; $-F=0.05 \mathrm{~mm}$. 


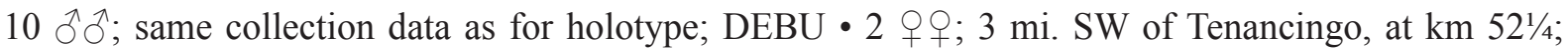

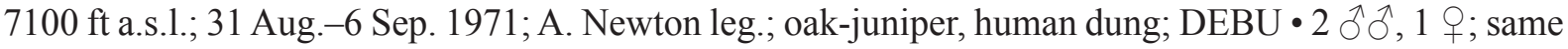
collection data as for preceding; UNAM. - Morelos • 1 क; 8 mi. S of Tres Cumbres; $7400 \mathrm{ft}$ a.s.1.; 29 Aug.-4 Sep. 1971; A. Newton leg.; oak/pine forest, dung; DEBU.

\section{Description}

BoDy. Length: 1.4-1.9 mm. Eye height $1.3 \times$ genal height. Head with 4 interfrontal setae and row of 8-10 small setulae on inner margin of orbital plate. Gena with 1 strong seta and 8 smaller setulae. Acrostichal setulae in 6-8 rows. Costagial seta extending to humeral break; second costal sector shorter than third (20:35); length of $\mathrm{M}_{1}$ between crossveins dm-cu and r-m 1.2 $\times \mathrm{dm}-\mathrm{cu}$; $\mathrm{CuA}_{1}$ stub vein $\sim 2.0 \times$

A
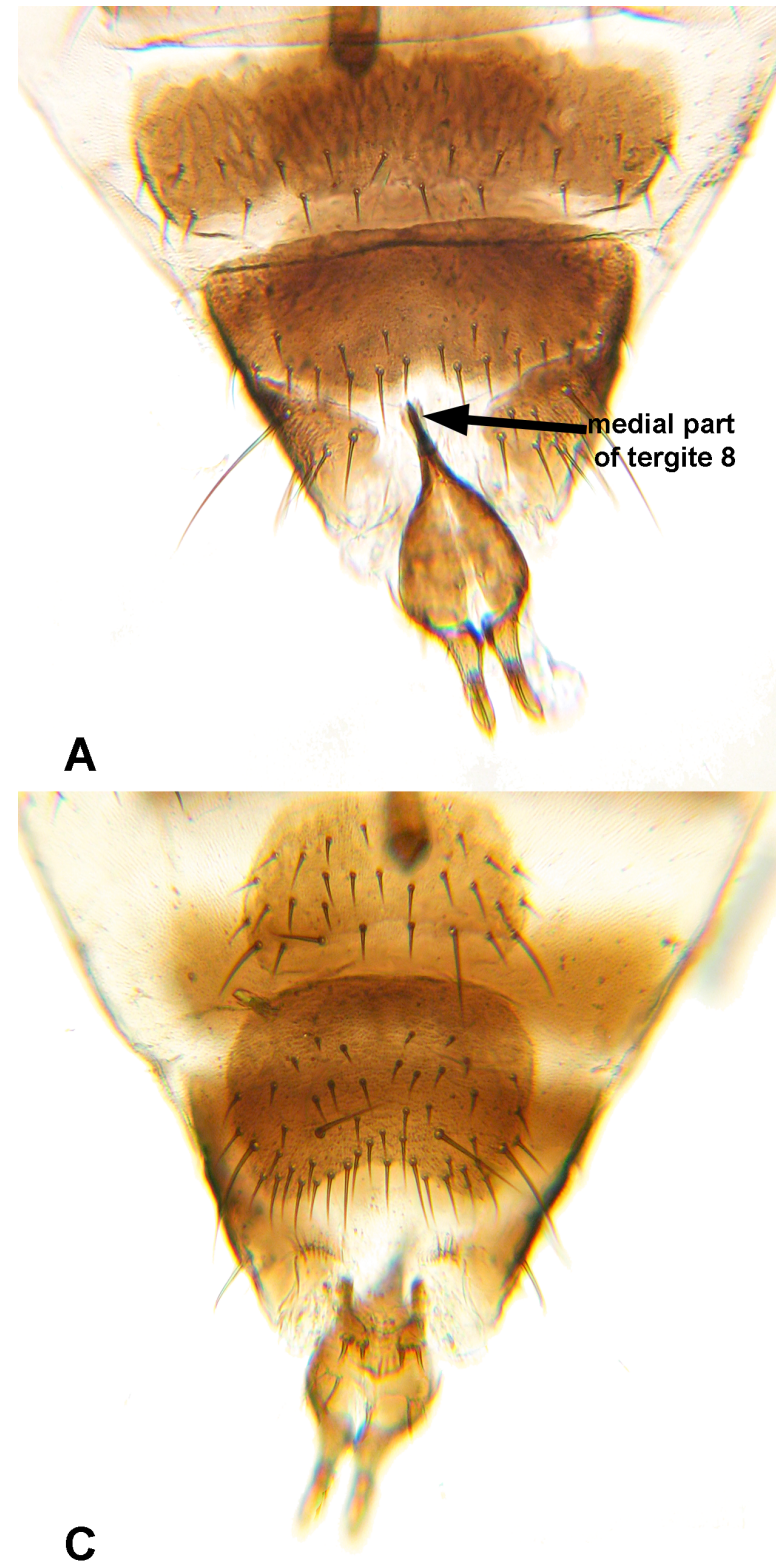

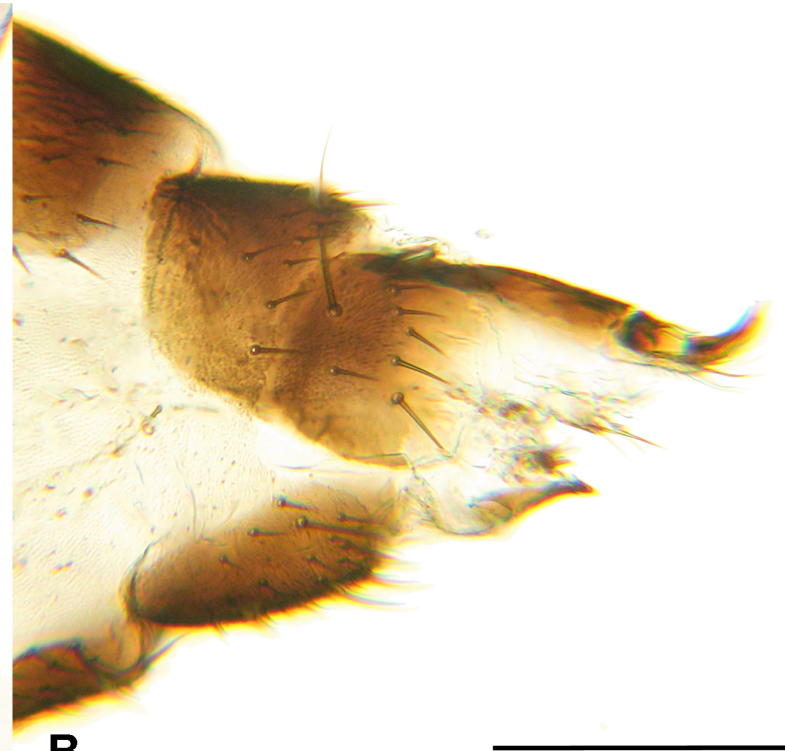

B

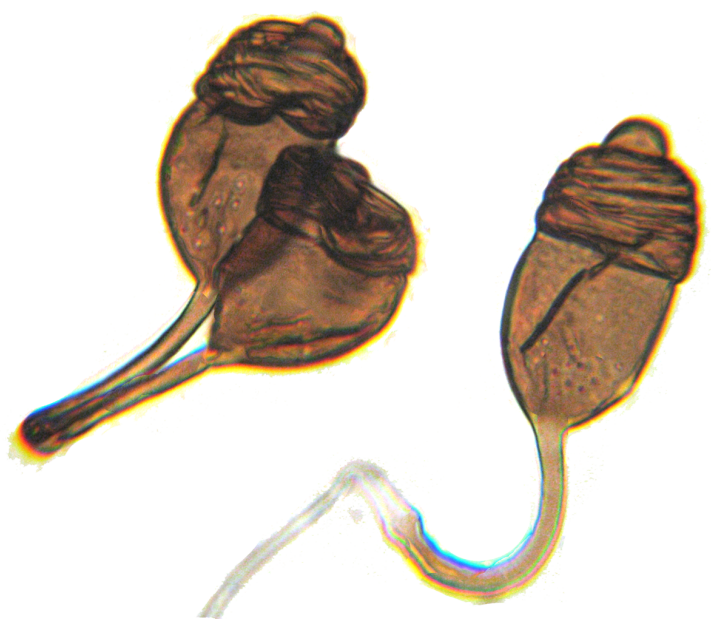

D

Fig. 17. Rudolfina pauca sp. nov., female terminalia (debu01086258). A. Terminal abdominal segments, dorsal view. B. Same as preceding, lateral view. C. Same as preceding, ventral view. D. Spermathecae. Scale bars: $\mathrm{A}-\mathrm{C}=0.20 \mathrm{~mm}$; $\mathrm{D}=0.05 \mathrm{~mm}$. 
$\mathrm{M}_{1}$ stub vein. Male mid femur with 4-5 weak setae in ventrobasal cluster. Male mid tibia with ventral comb of $11-13$ robust setae on apical $1 / 2$.

Male abdomen (Fig. 16). Sternites 3-4 evenly haired. Posterior margin of sternite 5 with lobes elongate, projecting posteriorly; emargination extending anteriorly $\sim 1 / 4$ length of sternite, $\sim 1 / 4$ width of sternite at posterior margin. Transverse part of sternite 6 weakly arcuate. Epandrium with 2 long ventrolateral setae and scattered setulae; subanal plate narrowly continuous below anal opening. Surstylus (in lateral view) trilobed, with anterior lobe rounded and bare, middle lobe small, rounded and with 4-6 distal setae apically, and posterior lobe posteriorly projecting and rounded, with 1 large, flattened seta and 2-3 regular setae apically. Cercus small and conical, projecting posterolaterally, with 2 apical setae. Postgonite slightly capitate or oar-shaped, with apex ventrally emarginate; 3-4 setulae present along dorsal surface. Dorsal sclerite of distiphallus (Fig. 16D-F) without swellings and not extending beyond apex of acrophallus.

Female abdomen (Fig. 17). Posterior margin of tergite 7 medially emarginate. Medial part of tergite 8 elongate, rectangular (length $\sim 3.0 \times$ width) and poorly sclerotized; overlapping basal projection of epiproct. Epiproct tulip-shaped, with elongate narrow anterior extension. Cercus reduced, shorter than apical flattened seta; fused anterolaterally to epiproct. Spermathecae acorn-shaped, with a small, smooth distal bulb, a wrinkled medial section and a smooth basal section; paired spermathecae with stems as long as spermatheca; common duct of pair short, $\sim 1 / 6$ length of stems.

\section{Remarks}

See comments under $R$. exuberata sp. nov.

Rudolfina pilosa sp. nov. urn:1sid:zoobank.org:act:8E664032-9FD5-4ED0-9A39-B63395A39655

Figs 18, 21C

\section{Etymology}

The species epithet is from the Latin for 'hairy', referring to the hirsute sternites 4 and 5 of the male.

\section{Material examined}

\section{Holotype}

MEXICO - đ'; Oaxaca, 2.0 mi. W of Jct. Mex. 175-Yuvila Rd; 9500 ft a.s.1.; 8-19 Aug. 1973; A. Newton leg.; oak-pine, dung; FMNH debu01086240.

\section{Paratypes}

MEXICO - Oaxaca • 1 đ̊; 6.6 mi. N of Ixtlan de Juarez; 8300 ft a.s.1.; 10-18 Aug. 1973; A. Newton leg.; oak woodland, human dung; DEBU 1 o ; $4.1 \mathrm{mi}$. W of Jct. Mex. 175-Yuvila Rd; $9300 \mathrm{ft}$ a.s.1.; 8-19 Aug. 1973; A. Newton leg.; oak-fir-pine forest; UNAM $\bullet 1$ \% ; same collection data as for preceding; oak-fir-pine forest; FMNH.

\section{Description}

Body. Length: 1.5-1.8 mm. Eye height $2.5 \times$ genal height. Head with 3 interfrontal setae and 5-6 small setae along inner margin of orbital plate. Gena with 2 strong setae and 7-9 smaller setulae. Acrostichal setulae in 4-6 rows. Costagial seta extending slightly beyond humeral break; second costal sector equal to third sector; length of $\mathrm{M}_{1}$ between crossveins dm-cu and r-m $2.0 \times \mathrm{dm}-\mathrm{cu}$; $\mathrm{CuA}$ stub vein $\sim 5.0 \times \mathrm{M}_{1}$ stub vein. Male mid femur with 4-5 small setae in ventrobasal cluster. Male mid tibia with ventral comb of 7-8 robust setae on apical $1 / 2$, midventral seta absent (female unknown). 

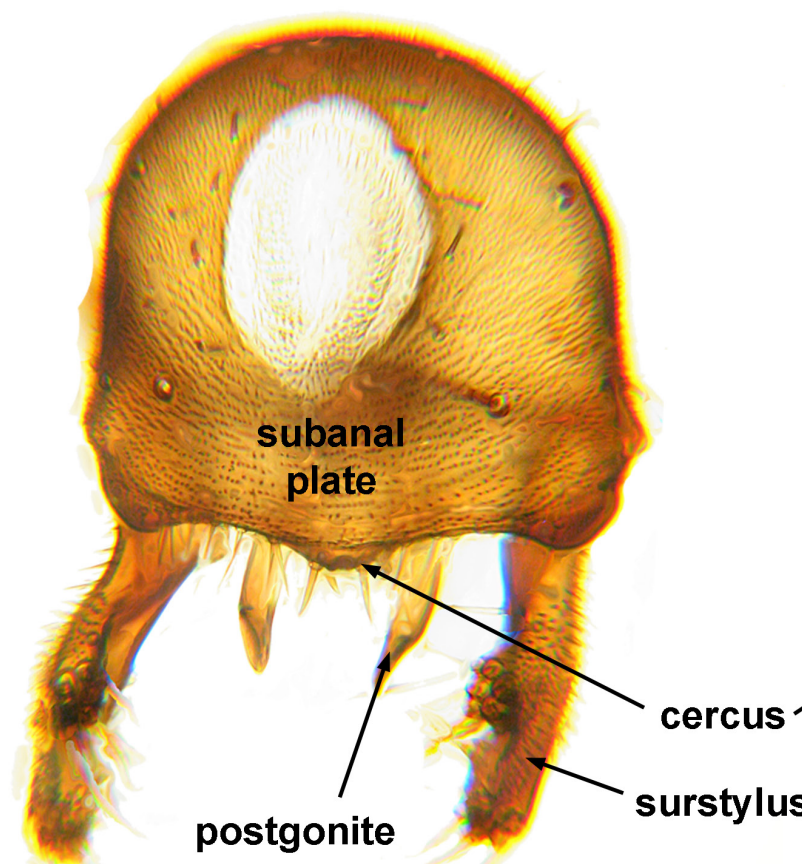

A
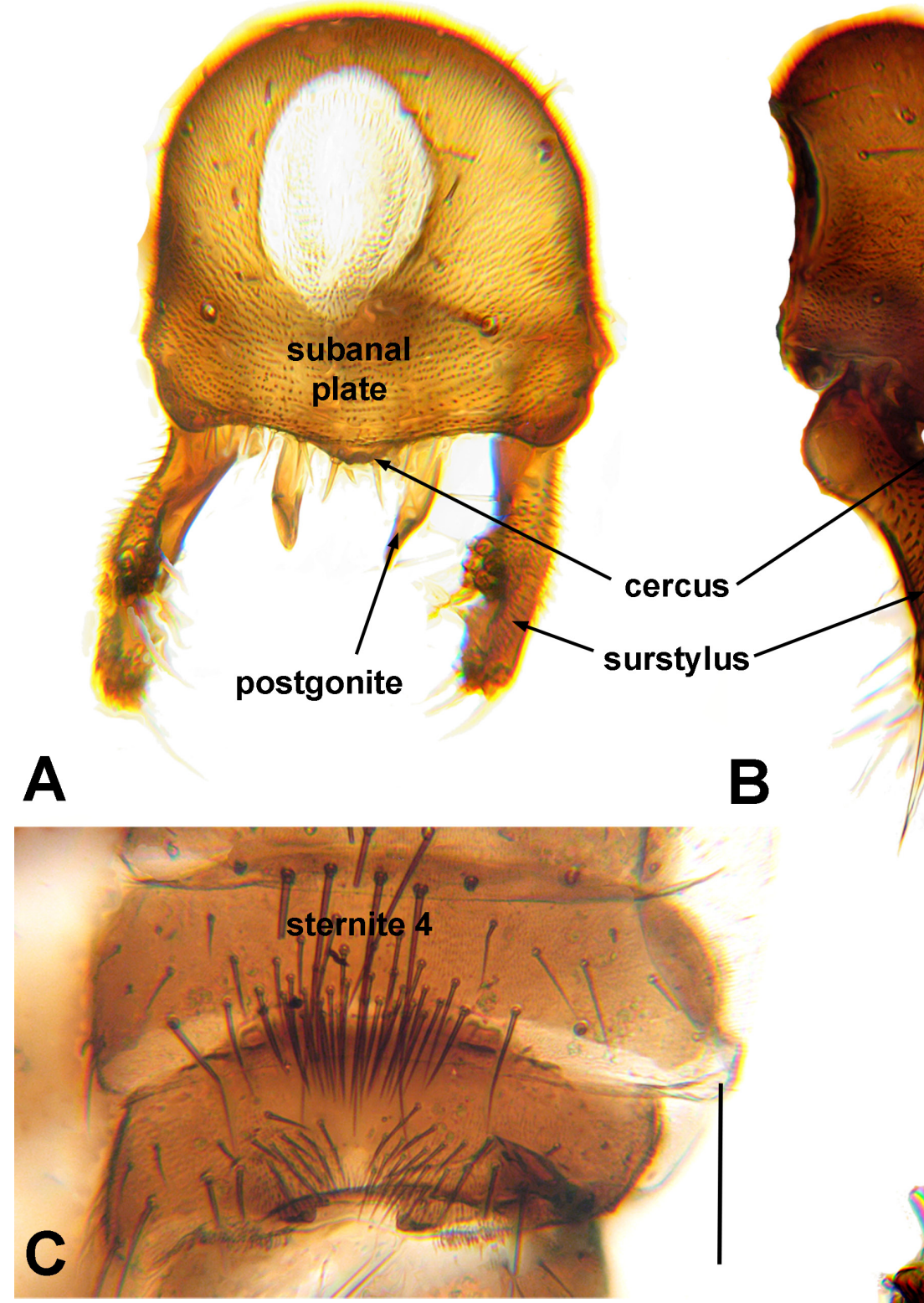

B

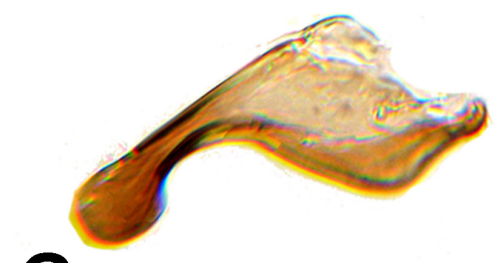

G

$\mathbf{F}$

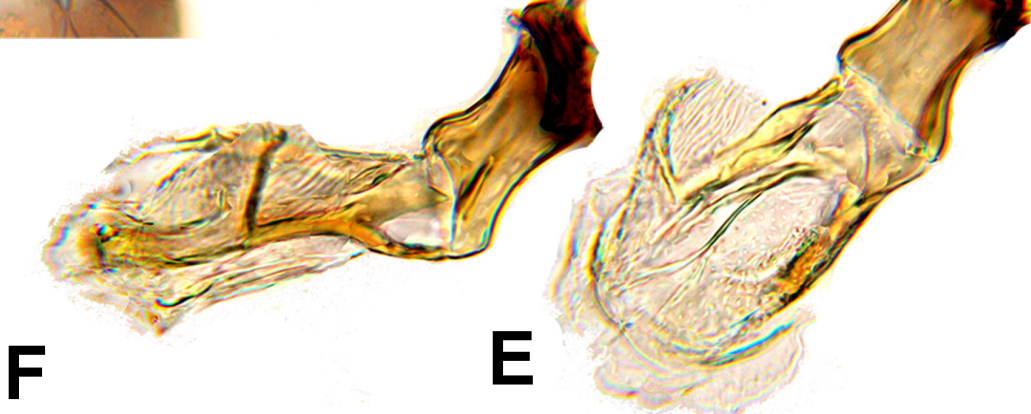

Fig. 18. Rudolfina pilosa sp. nov., male terminalia (debu01086241). A. Epandrium, surstylus and cercus, caudal view. B. Same as preceding, lateral view. C. Sternite 5, ventral view. D. Phallus and ejaculatory apodeme, dorsal view. E. Same as preceding, dorsolateral view. F. Same as preceding, lateral view. G. Postgonite, lateral view. Scale bars: $A-B=0.10 \mathrm{~mm} ; C=0.10 \mathrm{~mm} ; \mathrm{D}-\mathrm{G}=0.10 \mathrm{~mm}$. 
Male abdomen (Fig. 18). Sternite 3 and 4 with dense cluster of setae posteromedially. Posterior margin of sternite 5 with round teeth on each side of a medial emargination; emargination less than $1 / 6$ width of sternite and extending $\sim 1 / 2$ length. Transverse part of sternite 6 weakly arcuate. Epandrium with one large pair of setae ventrolaterally, one smaller pair of setae lateral to larger pair, and several setulae scattered over surface; subanal plate broadly continuous below anal opening. Surstylus boot-shaped, posteriorly bent near base, hirsute with 3-5 setae on posterior lobe (heel) and numerous smaller setae on anterior lobe (toe). Cercus fused with epandrium, with distinct seta on ventral margin. Postgonite clavate, with several setulae on dorsal surface. Dorsal sclerite of distiphallus (Fig. 18D-F) without distinct swellings and not extending beyond apex of acrophallus.

\section{Female}

Unknown.

\section{Remarks}

This species is most similar to $R$. newtoni sp. nov., which also has a setulose male sternite 4 and sternite 5 . These species are easily distinguished by the shape of the male surstylus.

Rudolfina remiforma sp. nov. urn:lsid:zoobank.org:act:01D37BA9-BF63-4930-9EF1-E1BB6D87C73F

Figs 19-20, 21C

\section{Etymology}

The species name is from the Latin for 'oar-shaped' and refers to the posterior lobe of the surstylus.

\section{Material examined}

\section{Holotype}

MEXICO • ${ }^{\text {; }}$ Chiapas, 21 mi. N of Bochil; $5500 \mathrm{ft}$ a.s.1.; 18-24 Aug. 1971; A. Newton leg.; pine, oak, Liquidambar, human dung; FMNH debu01086286.

\section{Paratypes}

MEXICO $\bullet 2$ 우; same collection data as for holotype; DEBU $\bullet 1$; same collection data as for holotype; FMNH.

\section{Description}

BoDy. Length: 1.4-1.8 mm. Eye height $2.0 \times$ genal height. Head with 4 interfrontal setae and row of 7 small setulae along inner margin of orbital plate. Gena with 1 strong seta and 8 smaller setulae. Acrostichal setulae in 4-6 rows. Costagial seta extending to humeral break; second costal sector shorter than third (20:65); length of $\mathrm{M}_{1}$ between crossveins dm-cu and $\mathrm{r}-\mathrm{m} 1.4 \times \mathrm{dm}-\mathrm{cu} ; \mathrm{CuA}_{1}$ stub vein $\sim 2.5-$ $3.0 \times \mathrm{M}_{1}$ stub vein. Male mid femur with 6 weak setae in ventrobasal cluster. Male mid tibia with ventral comb of $4-5$ robust setae on apical $1 / 3$.

Male abdomen (Fig. 19). Posterior margin of sternite 5 with small tooth on each side of medial emargination; emargination $\sim 1 / 3 \times$ width of sternite 5 . Transverse part of sternite 6 weakly arcuate. Epandrium regular, not swollen, with pair of long setae ventrolaterally and another 2-3 smaller pairs dorsolateral to longer pair; subanal plate narrow, incomplete, with narrow medial space. Surstylus with small anterior lobe and elongate posterior lobe; anterior lobe with 4-5 elongate setae apically; posterior lobe paddle-like and drawn out into rounded tip, with posterior margin weakly expanded near mid-length, anterior margin greatly expanded just before midlength, and 1 large seta near base. Cercus small and conical, projecting posteriorly; apical seta present. Postgonite simple, apex narrowly rounded. 


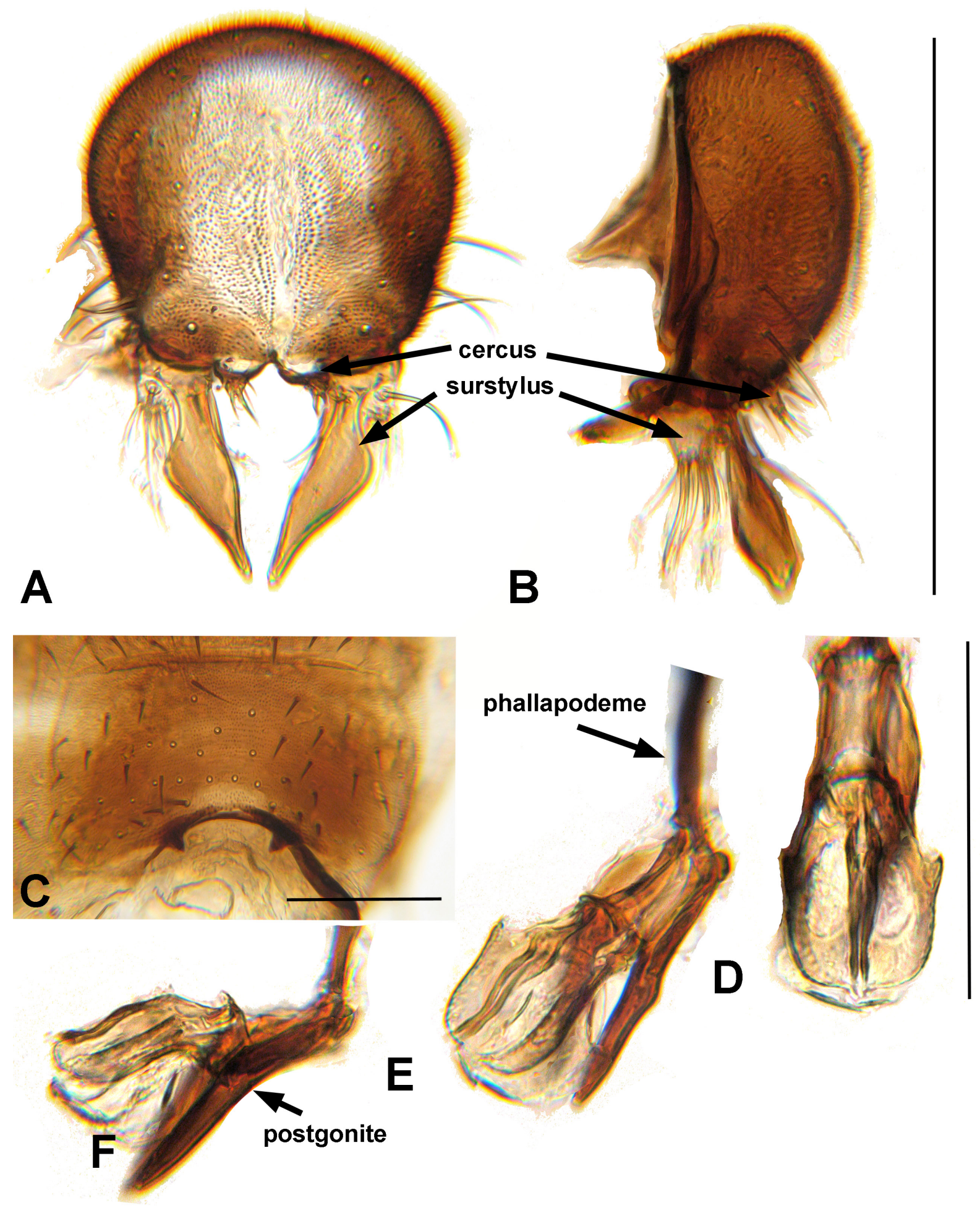

Fig. 19. Rudolfina remiforma sp. nov., male terminalia (debu01086286). A. Epandrium, surstylus and cercus, caudal view. B. Same as preceding, lateral view. C. Sternite 5, ventral view. D. Phallus and postgonites, dorsal view. E. Phallus, postgonites and phallapodeme, dorsolateral view. F. Same as preceding, lateral view. Scale bars: A-B $=0.25 \mathrm{~mm} ; \mathrm{C}=0.10 \mathrm{~mm}$; $\mathrm{D}-\mathrm{F}=0.08 \mathrm{~mm}$. 
Dorsal sclerite of distiphallus without distinct swellings and not extending distally beyond acrophallus (Fig. 19D-F).

Female abdomen (Fig. 20). Tergite 7 with posterior margin entire. Medial part of tergite 8 poorly sclerotized or fused with epiproct. Epiproct tulip-shaped, with length of anterior narrowed portion equal or subequal to that of posterior portion. Cercus small, as long as or only slightly longer than flattened apical seta; fused anterolaterally to epiproct. Sternite 7 with posterior margin broadly rounded. Spermathecae lenticular, inner surface with conical projection connecting with duct; paired spermathecae each with duct $\sim 2.0 \times$ length of spermatheca before common duct; single spermathecal duct similar in length.

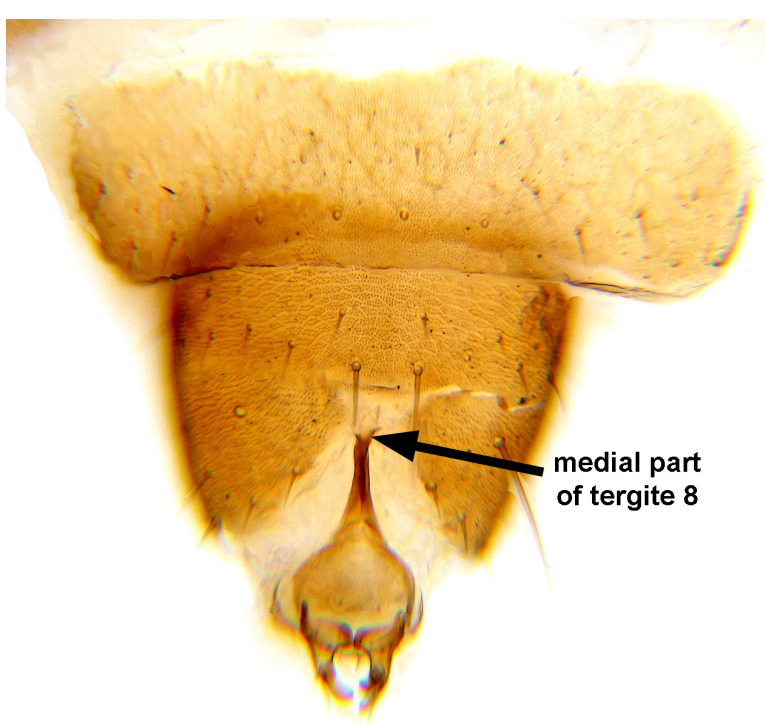

A

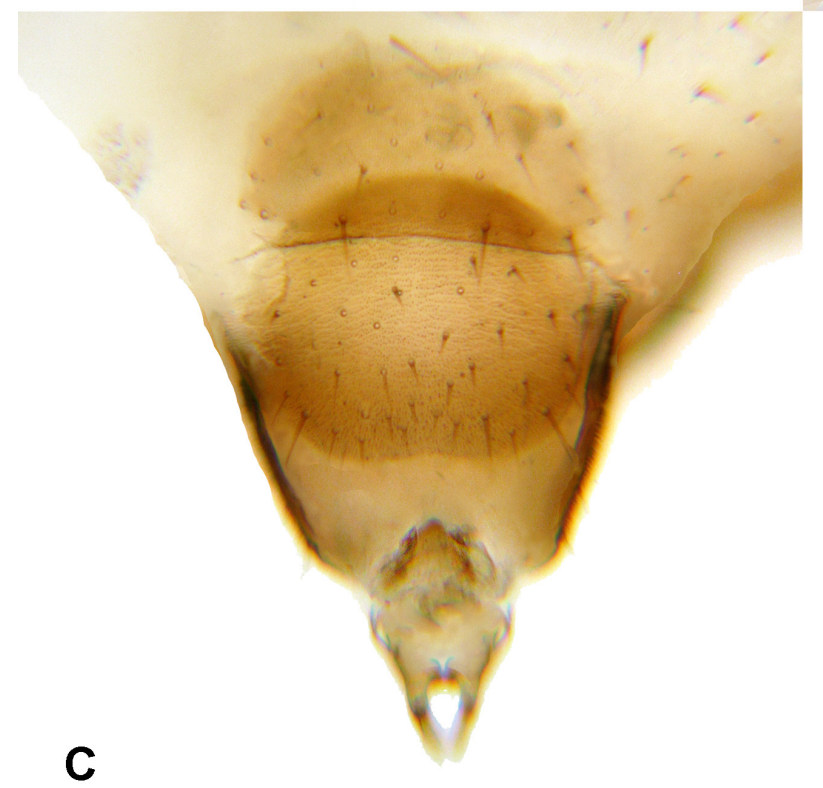

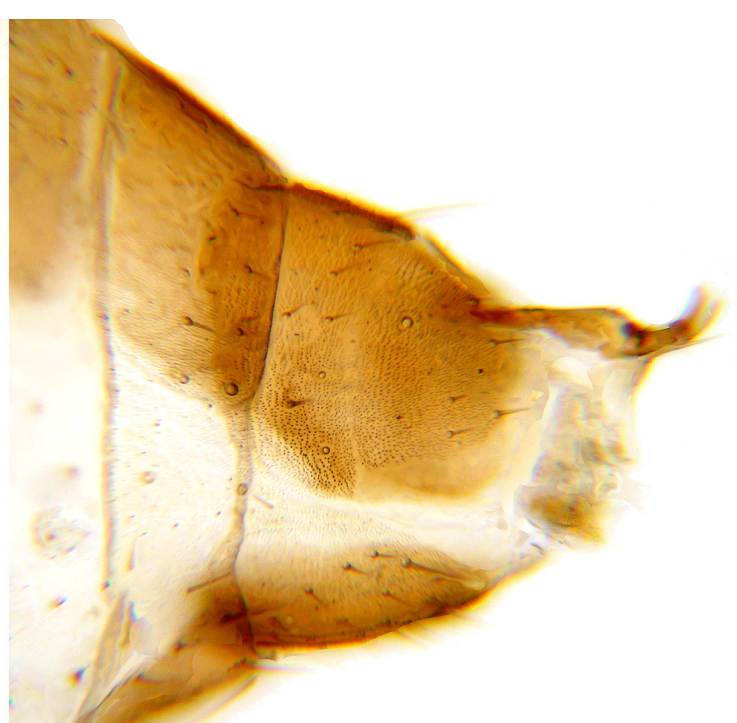

B

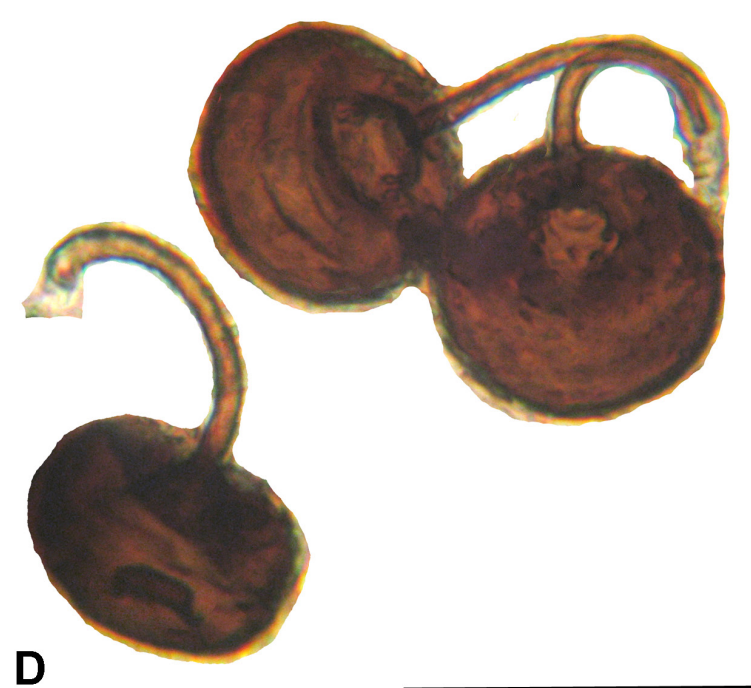

Fig. 20. Rudolfina remiforma sp. nov., female terminalia (debu01086288). A. Terminal abdominal segments, dorsal view. B. Same as preceding, lateral view. C. Same as preceding, ventral view. D. Spermathecae. Scale bars: A-C $=0.15 \mathrm{~mm} ; \mathrm{D}=0.05 \mathrm{~mm}$. 
PAIERO S.M. \& MARSHALL S.A., A revision of the genus Rudolfina Roháček (Sphaeroceridae)

\section{Remarks}

This species is closely related to R. exuberata sp. nov. and $R$. pauca sp. nov. and shares the modified epiproct found in those species.

Rudolfina rozkosnyi (Roháček, 1975)

Limosina rozkosnyi-Roháček, 1975: 119.

\section{Material examined}

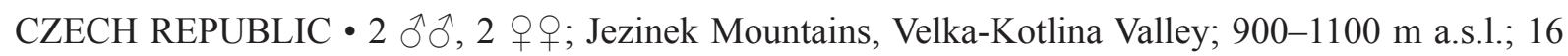
Aug. 1986; S.A. Marshall leg.; DEBU.

RUSSIA • 1 क; Siberia, Altai Reg., $\sim 50$ km SE of Teletskoya Lake; 1500 m a.s.1.; 13-15 Jul. 1991; S.A. Marshall leg.; wet area; DEBU.

\section{Description}

See Roháček (1975).

\section{Distribution}

Palaearctic: Austria, Czech Republic, Germany, Norway, Russia (North European Territory, West Siberia), Slovakia, Sweden.

\section{Remarks}

This species is here newly recorded from Siberia on the basis of a single female taken in pan traps near the margin of Teletskoya Lake. Previous Russian records are from the Northern European Territory.

Rudolfina tumida sp. nov. urn:lsid:zoobank.org:act:1FA40DBE-98EE-4665-A029-207ABCADE8DD

Figs 2-3, 21A

\section{Etymology}

The species epithet is from the Latin for 'swollen', referring to the enlarged epandrium.

\section{Material examined}

\section{Holotype}

UNITED STATES OF AMERICA • ${ }^{\text {; }}$; Wyoming, Uinta Co., 8 mi. SE of Evanston; $7100 \mathrm{ft}$ a.s.l.; 30 Jul.-11 Aug. 1979; S. and J. Peck leg.; sage-grass, riparian, carrion; DEBU debu01086083.

\section{Description}

Body. Length: 1.8-1.9 mm. Eye height $1.0 \times$ genal height. Head with 3 interfrontal setae and 5 small setae along inner margin of orbital plate. Gena with 2 strong setae and 4-5 smaller setulae. Acrostichal setulae in 4-6 rows. Costagial seta length unknown (broken on both sides of holotype); second costal sector shorter than third (35:40). Length of $\mathrm{M}_{1}$ between crossveins dm-cu and r-m $4.0 \times \mathrm{dm}-\mathrm{cu} ; \mathrm{CuA}_{1}$ stub vein $\sim 5.0 \times \mathrm{M}_{1}$ stub vein. Male mid femur basally with 9 strong setae present in ovoid cluster. Male mid tibia with ventral comb of $12-13$ robust setae on apical $2 / 3$.

MALE ABDOMEN (Figs 2-3). Posterior margin of sternite 5 with pair of small teeth on each side of shallow emargination; emargination extending $1 / 4$ length and $1 / 4$ width of sternite 5 . Transverse part of sternite 6 
weakly arcuate. Epandrium swollen, wider than preceding portion of abdomen (denuded on holotype); subanal plate incomplete. Posterior lobe of surstylus elongate, with apex and posterior margin heavily setose; anterobasal lobe broadly rounded and laminate, with distal triangular projection. Cercus ovoid. Postgonite elongate with apex acute. Dorsal sclerite of distiphallus (Fig. 21B-C) with several small swellings along length and extending beyond apex of acrophallus.

\section{Female}

Unknown.

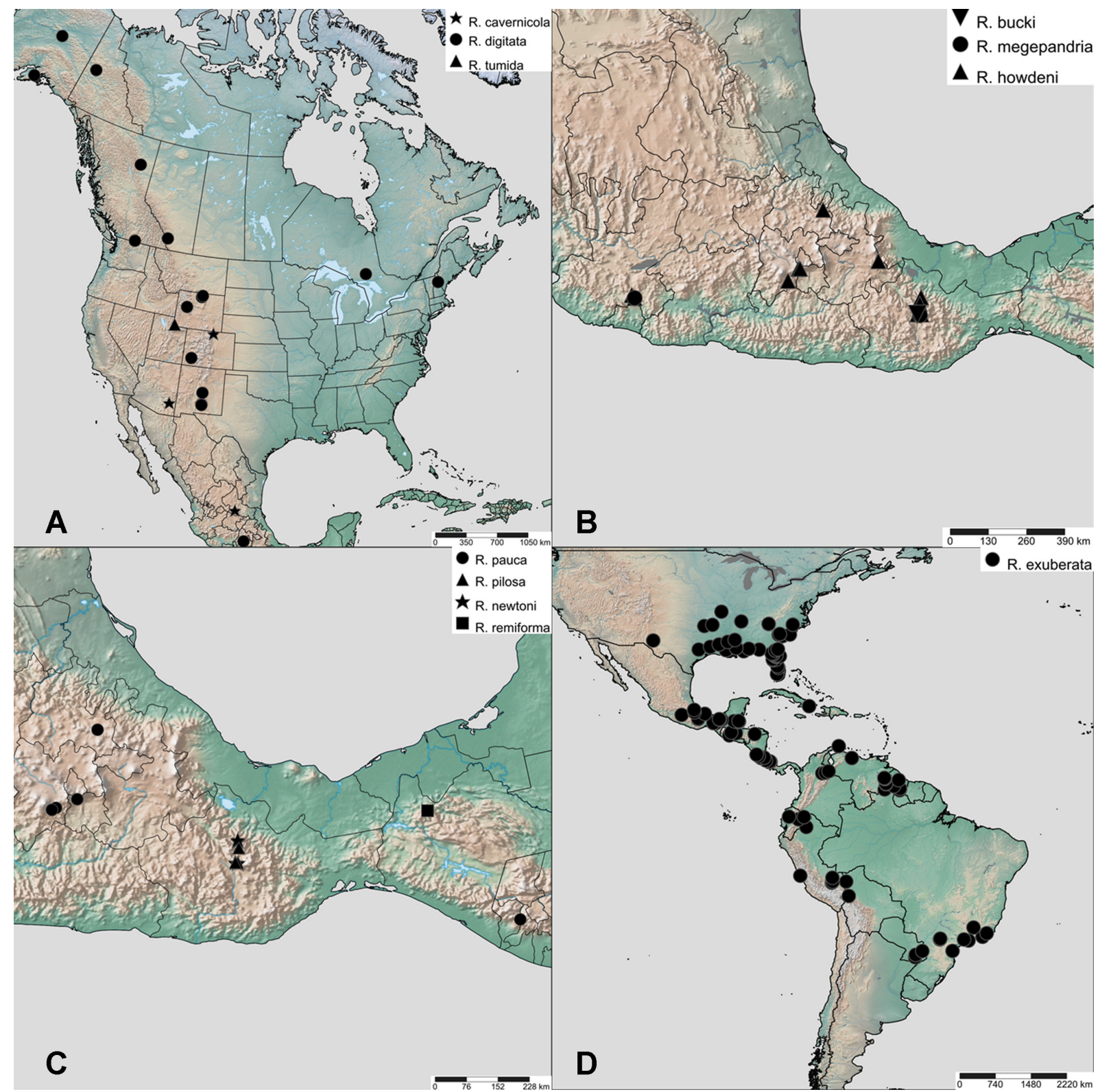

Fig. 21. Distribution of species of New World Rudolfina Roháček, 1987. A. R. cavernicola Marshall \& Fitzgerald, 1997, R. digitata Marshall, 1991 and R. tumida sp. nov. B. R. bucki sp. nov., R. megepandria sp. nov. and $R$. howdeni sp. nov. C. $R$. pauca sp. nov., $R$. pilosa sp. nov., $R$. newtoni sp. nov. and $R$. remiforma sp. nov. D. $R$. exuberata sp. nov. 
PAIERO S.M. \& MARSHALL S.A., A revision of the genus Rudolfina Roháček (Sphaeroceridae)

\section{Remarks}

Rudolfina tumida sp. nov. is distinctive for the uniquely swollen epandrium and large surstylus.

\section{Described species in other genera previously treated as Rudolfina}

Trachyopella opuntiae (Richards, 1967)

Leptocera (Trachyopella) opuntiae Richards, 1967: 531.

\section{Remarks}

Richards (1967) originally described this species as a Trachyopella Duda, 1918 (then a subgenus of Leptocera Olivier, 1813), a placement that was followed by Roháček \& Marshall (1986) in their review of Holarctic Trachyopella. Pitkin (1989) transferred opuntiae to Rudolfina, in view of its modified female cerci. Examination of type specimens, including male and female terminalia, supports the treatment of this species as a Trachyopella.

\section{Leptocera (Acuminiseta) prominens Duda, 1925}

Leptocera (Acuminiseta) prominens Duda, 1925: 124.

\section{Remarks}

Leptocera (Acuminiseta) prominens was provisionally treated as Rudolfina by Roháček et al. (2001), who noted that Acuminiseta Duda, 1925 does not occur in the New World. This species does not fit into Rudolfina as defined here, but belongs elsewhere in the Archiceroptera complex and will be treated in a later paper.

\section{Discussion}

With the exception of the widespread lowland species $R$. exuberata sp. nov., Rudolfina is a Holarctic, and largely Nearctic, genus with highest diversity in the highlands of Mexico. The few records from mountains in Mexico and Guatemala are from an area recognized as a transitional zone between the Nearctic and Neotropical regions, which has been referred to as the Mexican Transition Zone by some authors (e.g., Morrone 2014). The pattern of diversity in Rudolfina is consistent with those observed in some bats (Ortega \& Arita 1998), copepods (Aglaodiaptomus Light, 1938 and Skistodiaptomus Light, 1939; see Suarez-Morales et al. 2005), passalids (Gutierrez-Velazquez et al. 2013) and weevils (GarcíaNavarrete \& Morrone 2018). There are no records of Rudolfina species other than R. exuberata sp. nov. from lowland Guatemala, or any other Neotropical locality. We have studied tens of thousands of specimens collected from throughout Costa Rica over the past thirty years without finding any specimens other than $R$. exuberata sp. nov. that fit Rudolfina as currently defined; this reinforces our conclusion that the distribution of Rudolfina is effectively bounded southward by the limits of the Nearctic region. The origins of Rudolfina are likely to be in western North America, where R. cavernicola, the basalmost species, occurs, with the Nearctic and Palaearctic faunas diverging relatively early in Rudolfina's evolutionary history.

Sequence data for the 'barcode' region of cytochrome oxidase I was considered and supported species concepts consistent with the results presented here on the basis of morphology (Paiero 2017), but COI data was available for only three species ( $R$. digitata, $R$. rozkosnyi and $R$. exuberata sp. nov.). Other specimens of Rudolfina available at this time were mostly collected in pan traps and were not preserved adequately for genetic study. 
The number of new species in the University of Guelph insect collection and the apparent novelty of the limited material from other collections suggest that many further new species await discovery. Southern Mexico is especially poorly represented in the current material. We have only a single specimen (R. cavernicola) from the Sierra Madre Occidental range and we have seen no Rudolfina from the Sierra Madre Oriental. Further collecting efforts in Mexico are therefore likely to greatly improve our understanding of Rudolfina.

\section{Acknowledgements}

This work was made possible largely through the generosity of Dr. Alfred Newton (FMNH), who kindly allowed SAM to take the Sphaeroceridae from his Mexican residues during the 1980s. Of the 284 non- $R$. exuberata Rudolfina specimens studied for this revision, 219 were from these residues. Despite ongoing efforts to obtain further material from throughout the range of Rudolfina and related genera, the material collected by Dr. Newton during the early 1970s remains by far the most important collection of Mexican Rudolfina. Some of the drawings were prepared by Rebecca Langstaff. Funding for this study was provided by NSERC Discovery grants to SAM and NSERC postgraduate scholarships to SMP.

\section{References}

Cumming J.M. \& Wood D.M. 2010. Adult morphology and terminology. In: B.V. Brown (ed.) Manual of Central American Diptera, Volume 1: 9-63. NRC Research Press, Ottawa, Ontario.

García-Navarrete P.G. \& Morrone J.J. 2018. Testing the biogeographical regionalization of the Mexican Transition Zone based on the distribution of Curculionidae (Insecta: Coleoptera). Zootaxa 4530 (1): 1-99. https://doi.org/10.11646/zootaxa.4530.1.1

Goloboff P.A., Farris J. \& Nixon K. 2008. TNT: a free program for phylogenetic analysis. Cladistics 24: 774-786. https://doi.org/10.1111/j.1096-0031.2008.00217.x

Gutierrez-Velazquez A., Rojas-Soto O., Reyes-Castillo P. \& Halffter G. 2013. The classic theory of Mexican Transition Zone revisited: the distributional congruence patterns of Passalidae (Coleoptera). Invertebrate Systematics 27: 282-293. https://doi.org/10.1071/IS12056

Maddison W.P. \& Maddison D.R. 2017. Mesquite: a modular system for evolutionary analysis. Version 3.2. Available from https://www.mesquiteproject.org/ [accessed 29 Jan. 2017].

Marshall S.A. 1982. A Review of the Nearctic Limosininae (Diptera, Sphaeroceridae), with Revisions of Selected Genera. PhD thesis, University of Guelph, Canada.

Marshall S.A. 1991. Rudolfina digitata sp. nov., a new Nearctic sphaerocerid with a disjunct alpinearctic distribution. The Canadian Entomologist 123: 621-626. https://doi.org/10.4039/Ent123621-3

Marshall S.A. \& Buck M. 2010. Sphaeroceridae (Small dung flies). In: Brown B.V., Borkent A., Cumming J.M., Wood D.M., Woodley N.E. \& Zumbado M.A. (eds) Manual of Central American Diptera: 1165-1187. NRC Research Press, Ottawa, Ontario.

Marshall S.A. \& Cui Y. 2005. Systematics of Robustagramma, a new genus of New World Sphaeroceridae (Diptera). Zootaxa 1026: 1-122. https://doi.org/10.11646/zootaxa.1026.1.1

Marshall S.A. \& Fitzgerald S. 1997. Rudolfina cavernicola, a new species of cave-associated Sphaeroceridae (Diptera) from Colorado and Arizona. Proceedings of the Entomological Society of Washington 99: 641-644.

Marshall S.A. \& Richards O.W. 1987. Sphaeroceridae. In: McAlpine J.F., Peterson B.V., Shewell G.E., Teskey H.J., Vockeroth J.R. \& Wood D.M. (eds) Manual of the Nearctic Diptera, Part 2: 601-685. Agriculture Canada, Ottawa. 
PAIERO S.M. \& MARSHALL S.A., A revision of the genus Rudolfina Roháček (Sphaeroceridae)

Morrone J.J. 2014. Biogeographical regionalization of the Neotropical region. Zootaxa 3782 (1): 1-110. https://doi.org/10.11646/zootaxa.3782.1.1

Nixon K.C. 2002. WinClada ver. 1.00.08, published by the author, Ithaca, NY, USA.

Ortega J. \& Arita H.T. 1998. Neotropical-Nearctic limits in Middle America as determined by distributions of bats. Journal of Mammology 79 (3): 72-783. https://doi.org/10.2307/1383088

Paiero S.M. 2017. A Review of the Archiceroptera Papp Genus Complex (Diptera: Sphaeroceridae: Limosininae). $\mathrm{PhD}$ thesis, University of Guelph, Canada.

Pitkin B.R. 1989. A review of the Sphaeroceridae (Diptera) described by O.W. Richards. Occasional Papers on Systematic Entomology, London 6: 1-44.

Richards O.W. 1967. On a collection of Sphaeroceridae (Diptera) from the Galapagos Islands. Annals and Magazine of Natural History (Series 13) 9: 531-535.

Roháček J. 1975. Three new species of the genus Limosina Macq. (Sphaeroceridae, Diptera) from Czechoslovakia. Scripta Facultatis Scientiarum Naturalium Universitatis J.E. Purkynianae Brunensis, Biologia 2 (5): 115-126.

Roháček J. 1982. A monograph and reclassification of the previous genus Limosina Macquart (Diptera, Sphaeroceridae) of Europe, Part I. Beiträge zur Entomologie 32: 195-282.

https://doi.org/10.21248/contrib.entomol.32.2.195-282

Roháček J. 1983. A monograph and reclassification of the previous genus Limosina Macquart (Diptera, Sphaeroceridae) of Europe, Part II. Beiträge zur Entomologie 33: 3-195.

https://doi.org/10.21248/contrib.entomol.33.1.3-195

Roháček J. 1985. A monograph and reclassification of the previous genus Limosina Macquart (Diptera, Sphaeroceridae) of Europe, Part IV. Beiträge zur Entomologie 35: 101-179.

https://doi.org/10.21248/contrib.entomol.35.1.101-179

Roháček J. 1987. Replacement name for Rudolfia Roháček, 1982 (Diptera, Sphaeroceridae), with first record of R. rozkosnyi from northern Europe. Acta Entomologica Bohemoslovaca 84: 474-476.

Roháček J. 1998. Family Sphaeroceridae. In: Papp L. \& Darvas B. (eds) Contributions to a Manual of Palaearctic Diptera. Vol. 3., Higher Brachycera: 463-496. Science Herald, Budapest.

Roháček J. \& Marshall S.A. 1986. The genus Trachyopella Duda (Diptera, Sphaeroceridae) of the Holarctic Region. Monografie III. Museo Regionale di Scienze Naturali, Torino.

Roháček J., Marshall S.A., Norrbom A.L., Buck M., Quiros D.I. \& Smith I. 2001. World Catalog of Sphaeroceridae (Diptera). Slezské zemské muzeum, Opava, Czech Republic.

Shorthouse D.P. 2010. SimpleMappr, an online tool to produce publication-quality point maps. Available from http://www.simplemappr.net [accessed 29 Jan. 2017].

Smith I.P. \& Marshall S.A. 2004. A review of the New World genus Pterogramma Spuler and a revision of the Pterogramma sublugubrinum group (Diptera: Sphaeroceridae: Limosininae). Contributions in Science 499: 1-163.

Su L., Xu J. \& Cong G. 2017. A new species of the genus Rudolfina Roháček, 1987 (Diptera, Sphaeroceridae) from North-east China, with a key to the known Holarctic species of Rudolfina. Oriental Insects 51 (4): 391-396. https://doi.org/10.1080/00305316.2017.1315975 
Suarez-Morales E., Reind J.W. \& Elias-Guitierrez M. 2005. Diversity and distributional patterns of Neotropical freshwater copepods (Calanoida: Diaptomidae). International Review of Hydrobiology 90 (1): 71-83. https://doi.org/10.1002/iroh.200410742

Manuscript received: 13 June 2019

Manuscript accepted: 25 September 2019

Published on: 16 January 2020

Topic editor: Gavin Broad

Desk editor: Kristiaan Hoedemakers

Printed versions of all papers are also deposited in the libraries of the institutes that are members of the EJT consortium: Muséum national d'histoire naturelle, Paris, France; Meise Botanic Garden, Belgium; Royal Museum for Central Africa, Tervuren, Belgium; Royal Belgian Institute of Natural Sciences, Brussels, Belgium; Natural History Museum of Denmark, Copenhagen, Denmark; Naturalis Biodiversity Center, Leiden, the Netherlands; Museo Nacional de Ciencias Naturales-CSIC, Madrid, Spain; Real Jardín Botánico de Madrid CSIC, Spain; Zoological Research Museum Alexander Koenig, Bonn, Germany; National Museum, Prague, Czech Republic. 